\title{
Black hole binary remnant mass and spin: A new phenomenological formula
}

\author{
Carlos O. Lousto and Yosef Zlochower \\ Center for Computational Relativity and Gravitation, \\ School of Mathematical Sciences, Rochester Institute of Technology, \\ 85 Lomb Memorial Drive, Rochester, New York 14623
}

(Dated: September 25, 2018)

\begin{abstract}
We perform a set of 38 fully-nonlinear numerical simulations of equal-mass black-hole binaries in a configuration where the two black-hole spins in the binary are equal in both magnitude and direction, to study precession effects. We vary the initial direction of the total spin $\vec{S}$ with respect to the orbital angular momentum $\vec{L}$, covering the 2 dimensional space of orientation angles with 38 configurations consisting of 36 configurations distributed in the azimuthal angle $\phi$ and polar angle $\mu=\cos \theta$, and two configurations on the poles. In all cases, we set the initial dimensionless blackhole spins to 0.8 . We observe that during the late-inspiral stage, the total angular momentum of the system $\vec{J}$ remains within $5^{\circ}$ of its original direction, with the largest changes in direction occurring when the spins are nearly (but not exactly) counter-aligned with the orbital angular momentum. We also observe that the angle between $\vec{S}$ and $\vec{L}$ is nearly conserved during the inspiral phase. These two dynamical properties allow us to propose a new phenomenological formula for the final mass and spin of merged black holes in terms of the individual masses and spins of the progenitor binary at far separations. We determine coefficients of this formula (in the equal-mass limit) using a least-squared fit to the results of this new set of 38 runs, an additional set of five new configurations with spins aligned/counteraligned with the orbital angular momentum, and over a hundred recent simulations. We find that our formulas reproduce the remnant mass and spin of these simulations to within a relative error of $2.5 \%$. We discuss the region of validity of this dynamical picture for precessing unequal-mass binaries. Finally, we perform a statistical study to see the consequence of this new formula for distributions of spin-magnitudes and remnant masses with applications to black-hole-spin distributions and gravitational radiation in cosmological scenarios involving several mergers.
\end{abstract}

PACS numbers: 04.25.dg, 04.25.Nx, 04.30.Db, 04.70.Bw

\section{INTRODUCTION AND MOTIVATIONS}

The 2005 breakthroughs in Numerical Relativity [1 3 . allowed for accurate investigations of the orbital dynamics of merging black-hole binaries (BHBs) in the highlynonlinear regime between the slow inspiral (which can be modeled by post-Newtonian dynamics 4]) and the postmerger phase (which can be modeled by black-hole perturbation theory [5]). The current state-of-the-art simulations cover a broad range of astrophysical parameters, including mass ratios as small as $q=1 / 100$ [6, 7], highly spinning black holes with near maximal dimensionless spins $\alpha \leq 0.97$ 8 , and initial separations as large as $D=100 M$ [9].

Some of the most remarkable findings were related to effects of black-hole spin in the last stages of the merger. These include the hangup effect [10, which delays or expedites the merger depending on whether the spins are aligned or counter-aligned with the orbital angular momentum, and recoil velocities of several thousand $\mathrm{km} / \mathrm{s}$ [11 13. when the spins lie in opposite directions in the orbital plane. A configuration [14] combining both effects leads to even larger recoils, up to $5000 \mathrm{~km} / \mathrm{s}$.

Those effects were discovered by simulating highly symmetric configurations of equal-mass, nonprecessing BHBs. While it was possible to evolve generic configurations soon after the breakthrough [15, 16], it has not been until recently that systematic studies of precessing binaries have been performed [17 20]. In a recent paper, Ref. [21], we began the analysis and modeling of a set of runs of BHBs with equal-mass and equal-spin BHs (i.e., in magnitude and direction). These configurations, while symmetric, tend to maximize precession effects. Here, we provide extensive analysis of these and newer runs to model the evolution of the orbital angular momentum $\vec{L}$, the total spin of the system $\vec{S}$, and the radiation of the energy and angular momentum of the binary system.

The following are several highlights from our studies presented here. First, we find (see also Ref. [21]) that the angle between $\vec{L}$ and $\vec{S}$ varies by $\lesssim 2^{\circ}$ during the inspiral. This is a remarkable feature in a fully dynamical precessing binary that has immediate application to the modeling of the remnant since it connects parameters of the binary at large separation with those at merger, where most of the radiation takes place. While such a conservation of direction is indeed predicted [22, 23] by a lower-order post-Newtonian analysis, the degree to which this direction is conserved in the highly-dynamical (and highly-nonlinear) merger regime is remarkable. A second important observation for the modeling of our set of runs is the relatively small change in the direction of the total angular momentum $\hat{J}$, despite the significant continuous decrease in the magnitude of $\vec{J}$ as the binary evolves. Remarkably, in our runs we do not observe variations larger 
than $5^{\circ}$ in the direction of $\hat{J}$. This observation is consistent with the conclusions of 24, which where based on comparing initial and final directions of the angular momentum for a diverse set of fully nonlinear BHB simulations.

Our ultimate goal is to develop and test an empirical formula for the remnant mass and spin based on a Taylor expansion in terms of mass ratio and spins, as was done in [25, 26], but with different effective variables. Modeling the final spin and mass of the merger remnant is important for the creation of gravitational wave templates 27. to assist gravitational wave detectors, for studies of astrophysical scenarios involving merger of galactic black holes, and for modeling cosmological scenarios involving light versus heavy black holes seeds forming the structure of the universe [28, 29]. Importantly, our new formula reproduces the measured remnant mass and spin to within $0.1 \%$.

Here we will use the PN-inspired spin variables $\vec{S}=$ $\vec{S}_{1}+\vec{S}_{2}$ and $\vec{\Delta}=\left(M_{1}+M_{2}\right)\left(\vec{S}_{2} / M_{2}-\vec{S}_{1} / M_{1}\right)$, where $\vec{S}_{i}$ and $M_{i}$ are the spin and mass of $\mathrm{BH} i$ (note that [25, 26. base their expansion on $\vec{S}_{i}$ directly). We found when modeling the recoil velocity in [17] that $\vec{S}$ and $\vec{\Delta}$ are better suited as expansion variables than $\vec{S}_{1}$ and $\vec{S}_{2}$ because fewer terms are needed to get accurate fits. Our new formula represents a more comprehensive framework for modeling remnant quantities than our previous model 30, which made use of analytic expressions for the ISCO combined with numerical results available at the time (See also 31]). Other approaches to model remnants 32] also used lower-order spin expansions, and successive reasonable assumptions about the dynamics of spinning binaries 24, 33.

In this paper, we use the convention that $M_{i}$ is the $\mathrm{BH}$ mass as measured from a full numerical simulation and $m_{i}$ is the $\mathrm{BH}$ mass according to post-Newtonian (PN) theory. However, because it does not lead to confusion, and because we use the symbol $M$ to denote the unit of mass, we use $m$ to denote both the PN total mass $m=m_{1}+m_{2}$ and the full numerical total $\mathrm{BH}$ mass $m=M_{1}+M_{2}$. Note that in terms of dimensionless quantities

$$
\begin{aligned}
\frac{\vec{S}}{m^{2}} & =\frac{\vec{\alpha}_{2}+q^{2} \vec{\alpha}_{1}}{(1+q)^{2}}, \\
\frac{\vec{\Delta}}{m^{2}} & =\frac{\vec{\alpha}_{2}-q \vec{\alpha}_{1}}{1+q},
\end{aligned}
$$

where $q=M_{1} / M_{2}$ and $\vec{\alpha}_{i}=\vec{S}_{i} / M_{i}^{2}$. In addition, we use $M_{\text {rem }}$ and $\alpha_{\text {rem }}$ to denote the final remnant BH mass and dimensionless spin and

$$
\delta \mathcal{M}=\frac{M_{1}+M_{2}-M_{\text {rem }}}{M_{1}+M_{2}}
$$

to denote the fraction of the initial mass radiated by the binary. Finally, we denote unit vector in the direction of an arbitrary vector $\hat{v}$ with a hat (i.e., $\hat{v}=\vec{v} / v$ ).

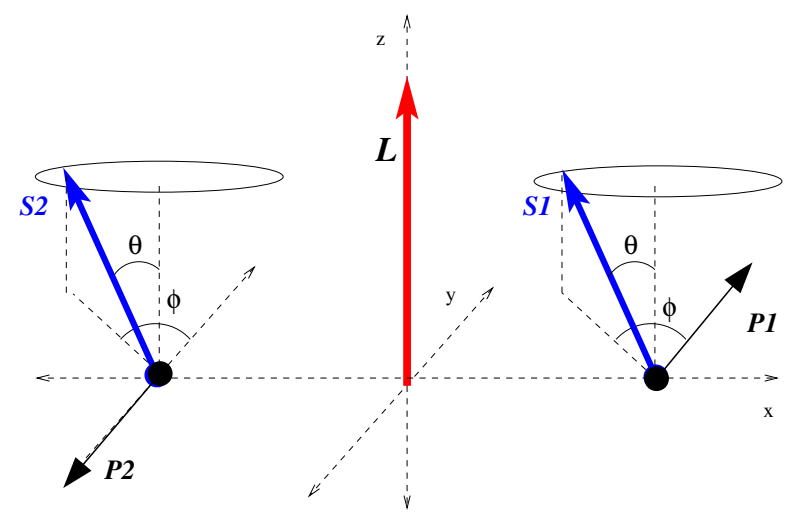

FIG. 1: Configuration of the SP precessing binary.

This paper is arranged as follows. In Sec. II] we give an overview of the numerical techniques used to generate initial data, evolve the BHBs, and measure the individual and remnant masses and spins. We also describe the initial configurations of the new BHB simulations presented here. In Sec. [II], we present the results from our BHB simulations described in Sec. II.

In Sec. IV, we develop the framework for modeling the final remnant mass and spin using $\mathrm{PN}$-inspired variables and symmetry considerations. In Sec. $\mathrm{V}$, we use a leastsquares fit of the remnant mass and spin of the new 38 precessing runs, 5 new non precessing runs, and those of Refs. 34 and 17 in the expansion formulas derived in Sec. IV. Our formulas are valid for the case of equal-mass binaries, and we provide an ansatz for extending them to the unequal-mass case.

In Sec. VI, to assess some astrophysical consequences of these new formulas, we find the distribution of spinmagnitudes after successive mergers (see also Ref. [35]) of similar-mass BHBs assuming randomly-oriented or partially-aligned spins, which are meant to model the effects of dry mergers, as well as mergers where accretion partially aligns the spins (note that we do not model the effects of accretion on the spin magnitudes) [34, 36, 37.

\section{NUMERICAL SIMULATIONS}

In order to single out the effects of precession we study BHBs consisting of two BHs with identical masses and spins (see Fig. 1). Two such configurations were first studied in [38, soon after the breakthroughs in numerical relativity. For a given spin-inclination angle, these configurations maximize precession effects, at least in the PN regime, because they maximize the total spin magnitude $S$. As seen in Eq. 12 below, $\dot{\vec{S}}(\vec{S}$ only changes due to precession at $2 \mathrm{PN}$ order) is proportional to $S$. In addition, the spin-orbit contribution to the radiation of angular momentum [see Eq. (3.28c) of Ref. [39]] is maximized for these configurations as well, since, in the equal-mass case, all terms in the formula for the radiation of angu- 
lar momentum are proportional to $S$. Note that, when the two spins are fully aligned or fully counteraligned with the orbital angular momentum, the BHB is nonprecessing [10. Also note that these configurations 38. are symmetric under parity [i.e. $(x, y, z) \rightarrow(-x,-y,-z)$ ] and, consequently, there is no recoil of the final remnants.

Our numerical simulations use the CACTUS/EinsteinToOlkit [40, 41] infrastructure and CARPET 42 mesh refinement driver, as well as both private and open-source thorns. We use the TwoPunctures thorn [43] to generate initial puncture data 44. for the BHB simulations. These data are characterized by mass parameters $m_{p}$ (which are not the horizon masses), as well as the momentum and spin, of each BH. We evolve these BHB data sets using the LAzEv 45. implementation of the moving puncture approach [2, 3] with the conformal function $W=\sqrt{\chi}=\exp (-2 \phi)$ suggested by Ref. [46. For the runs presented here, we use centered, eighth-order finite differencing in space 47 . and a fourth-order Runge - Kutta time integrator. Note that we do not upwind the advection terms.

We locate the apparent horizons using the AHFindERDIRECT thorn [48] and measure the horizon spin using the isolated horizon (IH) algorithm detailed in [49.

The families of BHBs considered here are equal-mass, equal-spins BHBs that are further characterized by three parameters, the initial orbital frequency, the polar inclination of the individual $\mathrm{BH}$ spins, and the azimuthal orientation of the spins. Due to the hangup effect, we choose larger initial orbital frequencies for the BHBs with small polar angles. For each polar angle $\theta$ we evolve a set of six azimuthal angles $\phi=0^{\circ}, 30^{\circ}, \cdots, 150^{\circ}$ (except for $\theta=0^{\circ}$ and $\theta=180^{\circ}$ ). We choose initial polar angles $\theta=0^{\circ}, 48.2^{\circ}, 70.5^{\circ}, 90^{\circ}, 109.5^{\circ}, 131.8^{\circ}, 146.4^{\circ}, 180^{\circ}$ (the polar angles were chosen to cover the sphere uniformly in $\cos \theta$, but with higher density near $\theta=180^{\circ}$ ). We denote these configurations by SPTHXXPHYY below, where $\mathrm{XX}$ is the polar angle (in degrees) and YY is the azimuthal angle. We also performed a high-resolution run of the SPTH0 configuration, which we denoted by SPTH0H below. For all the SP configurations, the dimensionless spin of each $\mathrm{BH}$ was $\alpha=0.8$. We chose this because it is relatively large, but can also be evolved relatively efficiently (spins of 0.85 require substantially more resources due to gauge effects).

In addition to the SP runs, we also evolve a set of equal-mass runs, with one $\mathrm{BH}$ spin aligned with the orbital angular momentum, and the other counter aligned. The two spins have the same magnitude. This type of configuration has been studied before in, e.g., [50, 51]. Here we are interested in measuring the radiated energy and final remnant spin. In previous modeling formulas, the effect of spin for this configuration was usually ignored [24, 33. We denote these configuration below by UD0.XX, where 0.XX gives the magnitude of the spin. We performed the UD simulations using 3 different resolutions to monitor the accuracy of the results. We denote these resolutions $\mathrm{C}, \mathrm{M}$, and $\mathrm{F}$ for coarse, medium, and fine, respectively. Here the coarse resolution corresponds to the resolution used for all SP configurations. The medium and fine results had 1.2 times and $1.2^{2}$ times the number of gridpoints in each direction. As seen in Fig 14, for a spin of $\alpha=0.8$, we can expect a relative error of $0.04 \%$ in the final remnant spin and $0.1 \%$ in the final remnant mass by using only the coarse resolution. Note also that the error increases dramatically when the spin is increased to $\alpha=0.85$.

For the computation of the radiated angular momentum components, we use formulas based on "fluxlinkages" [52] and explicitly written in terms of $\psi_{4}$ in [53, 54].

From the waveform extracted at a given radius $R$ (i.e. the extraction sphere), we calculate the rate with which angular momentum is radiated out of the extraction sphere to infinity, which we denote with the symbol

$$
\frac{d}{d t}\left(\overrightarrow{\delta J}_{\mathrm{rad}}(t)\right)
$$

and the net angular momentum radiated through the extraction sphere from the initial slice to time $t$, which is given by

$$
\overrightarrow{\delta J}_{\mathrm{rad}}(t)=\int_{0}^{t} \frac{d}{d \tau}\left(\overrightarrow{\delta J}_{\mathrm{rad}}(\tau)\right) d \tau .
$$

We then define the total angular momentum at time $t$ to be

$$
\vec{J}(t)=J_{\mathrm{ADM}}-\overrightarrow{\delta J}_{\mathrm{rad}}(t),
$$

which is the total angular momentum contained on slice $t$ within the extraction sphere. Because of the finite propagation speed of the radiation, when comparing local and radiative techniques for the angular momentum, we translate the radiative quantities in time by $R$, which is a crude, but accurate enough, measure of the propagation time.

For the current paper, it is important to measure the direction of the orbital angular momentum. To do this we use a few different techniques. First, we define $\vec{L}$ via

$$
\begin{array}{r}
\vec{L}(t)=\vec{J}(t)-\vec{S}(t), \\
\hat{L}(t)=\frac{\vec{L}(t)}{|\vec{L}(t)|},
\end{array}
$$

Here $\vec{S}(t)=\vec{S}_{1}(t)+\vec{S}_{2}(t)$ is the sum of the spins of the individual BHs, as measured by the IH formalism (its direction is inferred from the zeros of the approximate Killing field [38]).

We can also define a purely coordinate based measure of the orbital angular momentum direction using the coordinate trajectory $\vec{r}(t)$, given by

$$
\hat{L}_{\text {coord }}=\frac{\vec{r}(t) \times \dot{\vec{r}}(t)}{|\vec{r}(t) \times \dot{\vec{r}}(t)|} .
$$


Note that while this equation resembles the Newtonian definition of angular momentum, we apply it to fully nonlinear numerical trajectories, thus including relativistic corrections. One could also use an alternative definition in terms of the quasilocal linear momentum $\vec{p}$, as was proposed in [55].

In the text below, when needed for clarity, we refer to $\hat{L}(t)$ as the radiation angular momentum and $\hat{L}_{\text {coord }}(t)$ as the coordinate angular momentum.

We also compare $\hat{L}(t)$ and $\hat{L}_{\text {coord }}(t)$ with the purely radiation-based measures of the preferred asymptotic frames of O'Shaughnessy et al. [56 and Boyle [57. Briefly, these two are based on the symmetric matrix $\left\langle f\left|\mathbf{J}_{(a} \mathbf{J}_{b)}\right| f\right\rangle\left[{ }_{(a} b\right)$ denotes symmetrization over the two indices] and vector $\Im\left(\left\langle f\left|\mathbf{J}_{a}\right| \partial_{t} f\right\rangle\right)$, where $\mathbf{J}_{a}$ is the angular momentum operator and $f$ is a waveform-related function, e.g. the strain $h$, the Bondi News $N$, or the Newman-Penrose scalar $\psi_{4}$, and $\Im(z)$ denotes the imaginary part of $z$. The angular momentum operator for spin-weighted functions is given by [58] 69.

$$
\begin{aligned}
& \mathbf{J}_{x}=i \sin \phi \partial_{\theta}+\cos \phi\left(i \cot \theta \partial_{\phi}+s \csc \theta\right) \\
& \mathbf{J}_{y}=-i \cos \phi \partial_{\theta}+\sin \phi\left(i \cot \theta \partial_{\phi}+s \csc \theta\right) \\
& \mathbf{J}_{z}=-i \partial_{\phi}
\end{aligned}
$$

where $s$ is the spin-weight. Note that the radiated angular momentum is given by 53,58

$$
\frac{d}{d t}\left(\overrightarrow{\delta J}_{\mathrm{rad}}(t)\right)=\lim _{r \rightarrow \infty} \frac{r^{2}}{16 \pi} \Im\left\langle h\left|\mathbf{J}_{a}\right| \partial_{t} h\right\rangle .
$$

O'Shaughnessy et al. define the preferred frame to be one with $z$ axis aligned with the eigenvector corresponding to the largest eigenvalue of $\left\langle f\left|\mathbf{J}_{(a} \mathbf{J}_{b)}\right| f\right\rangle$ and Boyle defines it to be the frame aligned with the vector $\left\langle f\left|\mathbf{J}_{(a} \mathbf{J}_{b)}\right| f\right\rangle^{-1}\left(\Im\left\langle f\left|\mathbf{J}_{a}\right| \partial_{t} f\right\rangle\right)$. For this work we explore using the choices $f=\psi_{4}$, the Bondi News $N$, and the strain $h$ (note that Boyle defines $f=h$ and with this choice is able to recover the post-Newtonian $\vec{L}$ ). We denote the directions defined by O'Shaughnessy et al. by $\vec{O}_{X}$, where $X$ is $\psi, N$, or $h$, corresponding to using $f=\psi_{4}, f=N$, and $f=h$, respectively. Similarly, we denote the preferred direction of Boyle by $\vec{B}_{X}$, where $X$ is $N$ or $h$, corresponding to $f=N$ and $f=h$.

\section{RESULTS}

\section{A. Results from the SP configurations}

Here we present results relating to the precession of the SP configurations. Detailed tables with the initial data parameters, radiated energy-momentum, and remnant properties can be found in Appendix A.

For all the SP configurations, we find that, while $\vec{J}$ changes in magnitude by more than $30 \%$, the direction of $\vec{J}$ is largely unchanged during the entire simulation.

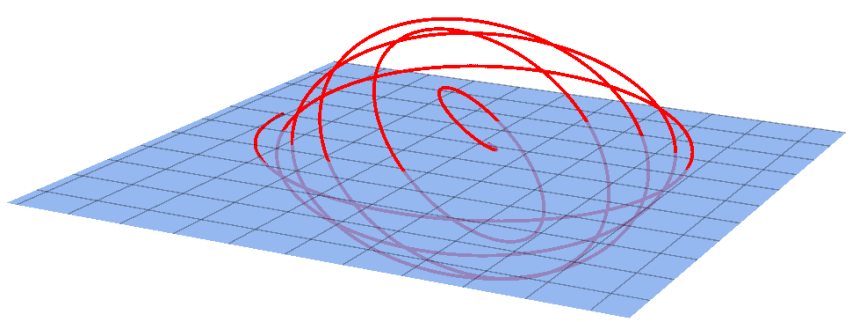

FIG. 2: The coordinate trajectory for a typical SP configuration.

TABLE I: The change in the direction and magnitude of $\vec{J}$ for configurations with different inclination $(\theta)$ angles. Here $\{\vec{A}, \vec{B}\}=\cos ^{-1}(\hat{A} \cdot \hat{B})$ measures the angle between two vectors.

\begin{tabular}{lllllll}
\hline \hline $\cos \theta$ & $2 / 3$ & $1 / 3$ & 0 & $-1 / 3$ & $-2 / 3$ & $-5 / 6$ \\
\hline $\max _{t \phi}\{\hat{J}(t), \hat{J}(0)\}$ & $0.87^{\circ}$ & $1.40^{\circ}$ & $2.31^{\circ}$ & $2.97^{\circ}$ & $4.31^{\circ}$ & $4.25^{\circ}$ \\
$\max _{t \phi}\{\widehat{\delta J}(t), \hat{J}(t)\}$ & $15.68^{\circ}$ & $21.53^{\circ}$ & $25.18^{\circ}$ & $27.39^{\circ}$ & $27.12^{\circ}$ & $23.81^{\circ}$ \\
\hline \hline
\end{tabular}

We thus only observe simple precession in these equalmass BHBs. For convenience, we introduce the notation $\{\vec{A} \cdot \vec{B}\}=\cos ^{-1}(\hat{A} \cdot \hat{B})$ for the angle between two vectors. As seen in Table $\mathrm{I}$ and Fig 3 , the net rotation of $\hat{J}$ (i.e. the maximum of $\{\vec{J}(t), \vec{J}(0)\})$ is under $5^{\circ}$. The table also shows how far $\overrightarrow{\delta J}_{\text {rad }}(t)$ deviates from $\hat{J}$ $\left(\left\{\overrightarrow{\delta J}_{\text {rad }}(t), \vec{J}(t)\right\}_{\max }\right)$ for selected runs.

Figure 2 shows a typical trajectory $\left[\vec{r}(t)=\vec{r}_{1}(t)-\right.$ $\vec{r}_{2}(t)$ ], while Fig. 3 , shows the precession of $\vec{J}(t)$ for all $\mathrm{SP}$ configurations. For a given configuration, the maximum precession occurs near merger, with $\vec{J}(t)$ moving back towards its initial location during the plunge. This antiprecession effect is most noticeable for smaller values of $\theta$. The figure also shows the real part of the $(\ell=2, m=2)$ mode of $\psi_{4}$ to provide a reference time scale. By comparing $\psi_{4}$ with $\vec{J}(t)$, we can see that the antiprecession occurs roughly at merger. As shown in Fig. 4 and Table $\mathrm{I}$, the maximum deviation of $\vec{J}(t)$ from its initial orientation is a strong function of $\theta$. Note how the precession of $\vec{J}(t)$ increases as the polar angle $\theta$ increases until about $\theta=140^{\circ}$, where it then must rapidly decrease to zero at $\theta=180^{\circ}$ (which is a non-precessing configuration).

The reason why the maximum deviation of $\vec{J}$ from its initial orientation occurs when $\vec{S}$ is nearly counteraligned with $\vec{L}$ is in part due to the smaller magnitude of $\vec{J}$ for anti-aligned configurations, which makes it easier to tilt $\vec{J}$ even though the anti-aligned configurations radiate less than the aligned ones. In the maximally spinning case, the largest precession of $\vec{J}$ should occur when the spins are even closer to full counteralignment (see Sec. IV B).

As shown in Fig. 5 the magnitude of the individual $\mathrm{BH}$ spins is conserved to a very high degree during the 


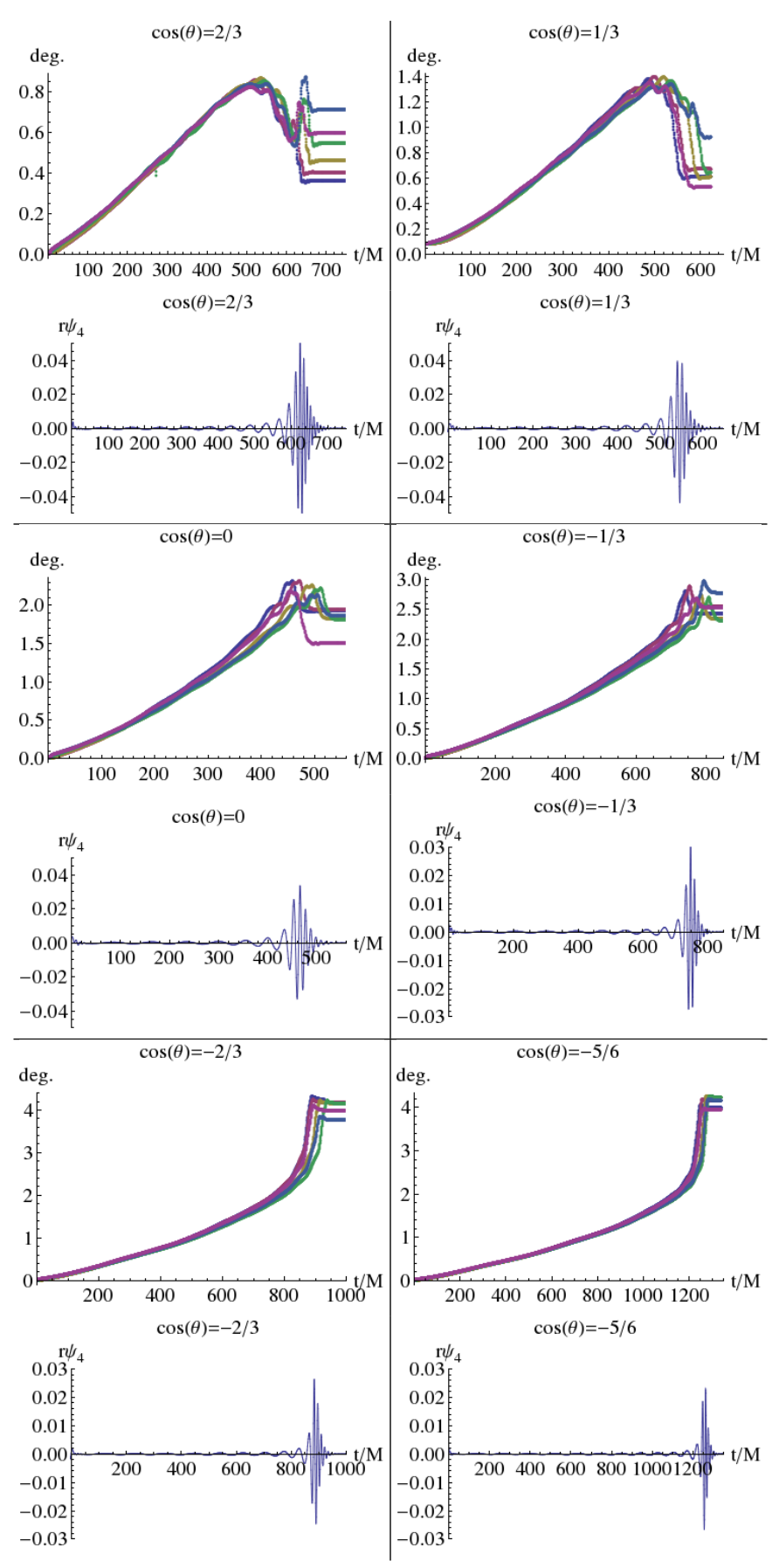

FIG. 3: The precession of $\hat{J}$ (compared to its initial direction) as a function of time for the SPTH48 (top left), SPTH70 (top right), SPTH90 (middle left), SPTH110 (middle right), SPTH132 (bottom left), and SPTH146 (bottom right) configurations. The lower plots show the $(\ell=2, m=2)$ mode of $\psi_{4}$ for the same time scale. Note that the precession angle decreases during the large burst of radiation at merger. Each azimuthal configuration (for a given inclination angle) is shown in a different color to give an idea of how the final angle varies with the initial azimuthal angle.

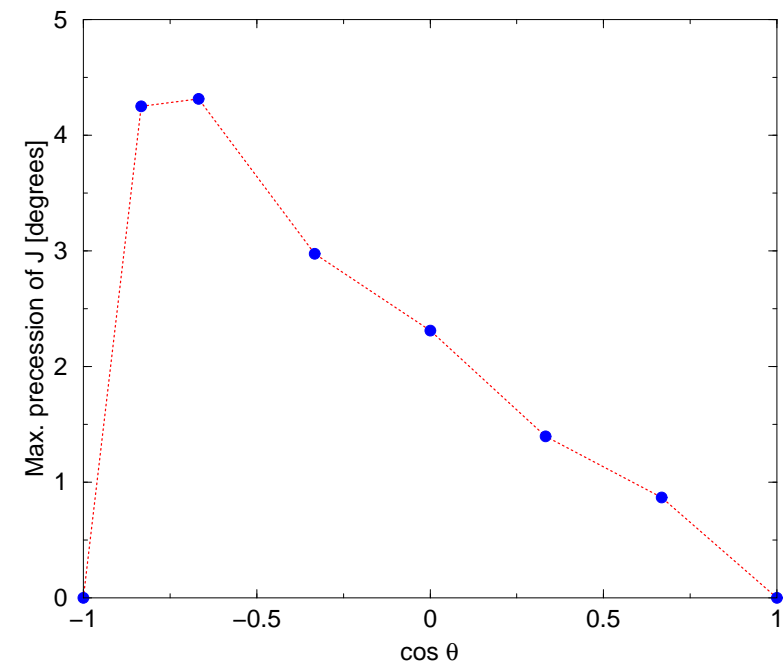

FIG. 4: The maximum deviation of $\vec{J}$ from its initial orientation versus $\theta$. Here the maximum is over time and over all $\phi$ configurations for a given $\theta$ configuration.

simulation. The total angular momentum $\vec{J}$, however, is not. Indeed the fraction of angular momentum lost to radiation during an inspiral approaches 1 as the initial separation approaches infinity. We interpret this to mean that orbital angular momentum is lost to radiation, while spin angular momentum is largely conserved. Note that, for the configurations studied here, the total spin must also be conserved in magnitude because the two spins cannot rotate with respected to each other, as that would break parity symmetry.

When $\vec{J}$ exhibits simple precession, as in our simulations, even though its magnitude is always dominated by the highly precessing $\vec{L}$, the spins of the individual BHs are always oriented such that the direction of $\vec{J}$ is largely unchanged. Thus the direction of the spin of the merger remnant $\vec{S}_{\text {rem }}$ agrees very closely with that of $\vec{J}_{\text {initial }}$ (which was also observed by Barausse et al. 24]).

Since the spin magnitude is conserved to a very high degree, and the binary radiates angular momentum continuously, it is not surprising that the angle between the total spin $\vec{S}(t)$ and $\vec{J}(t)$ decreases secularly, as shown in Fig. 6. From this, we can conclude that the component of $\vec{S}$ along $\vec{J}$ is not conserved on secular timescales. On the other hand, as seen in Fig. 7 the angle between $\vec{S}(t)$ and $\vec{L}(t)$, as defined using Eq. (2), shows no noticeable secular trend. That is to say, the average (over an orbital timescale) of $\hat{L} \cdot \hat{S}$ is nearly constant (in PN theory, it is the orbit average of $\hat{L} \cdot \vec{S}$ that is approximately conserved too). From this, and the fact that $|\vec{S}(t)|$ is conserved, we can conclude that, on average, the component of $\vec{S}$ along $\vec{L}(t)$ is conserved. Finally, in Fig. 8. we show that the angle between $\vec{L}(t)$ and $\vec{J}(t)$ increases on secular timescales. 


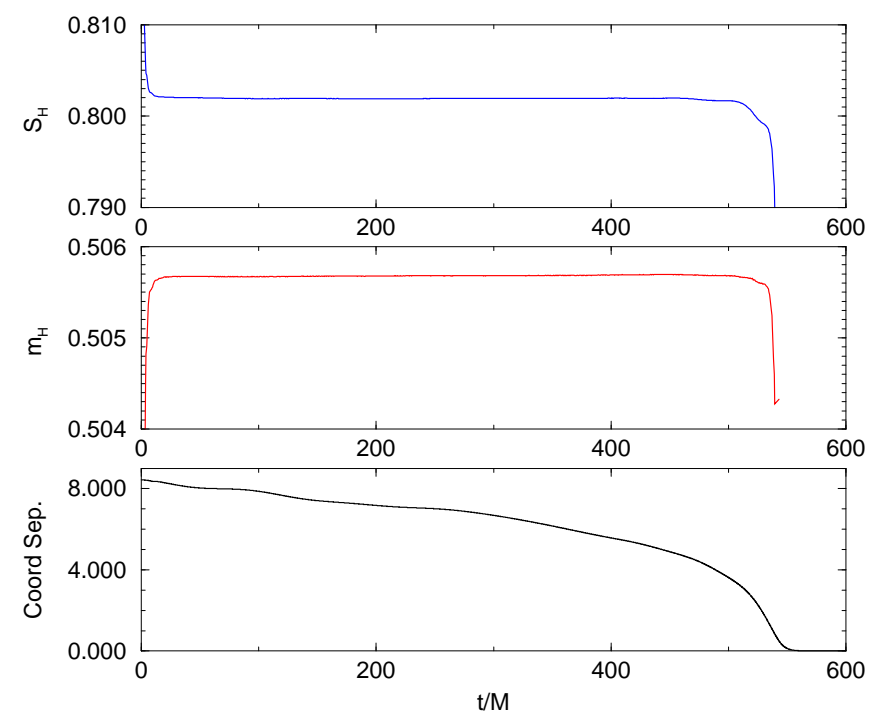

FIG. 5: The individual horizon spin angular momentum (not rescaled by the horizon mass), horizon mass, and coordinate separation of the two BHs for a prototypical simulation (SPTH70PH0). Note how the spin magnitude (and horizon mass) is conserved over the entire inspiral until just before merger. At merger, the isolated horizon approximation, which measures the spins, is no longer valid.
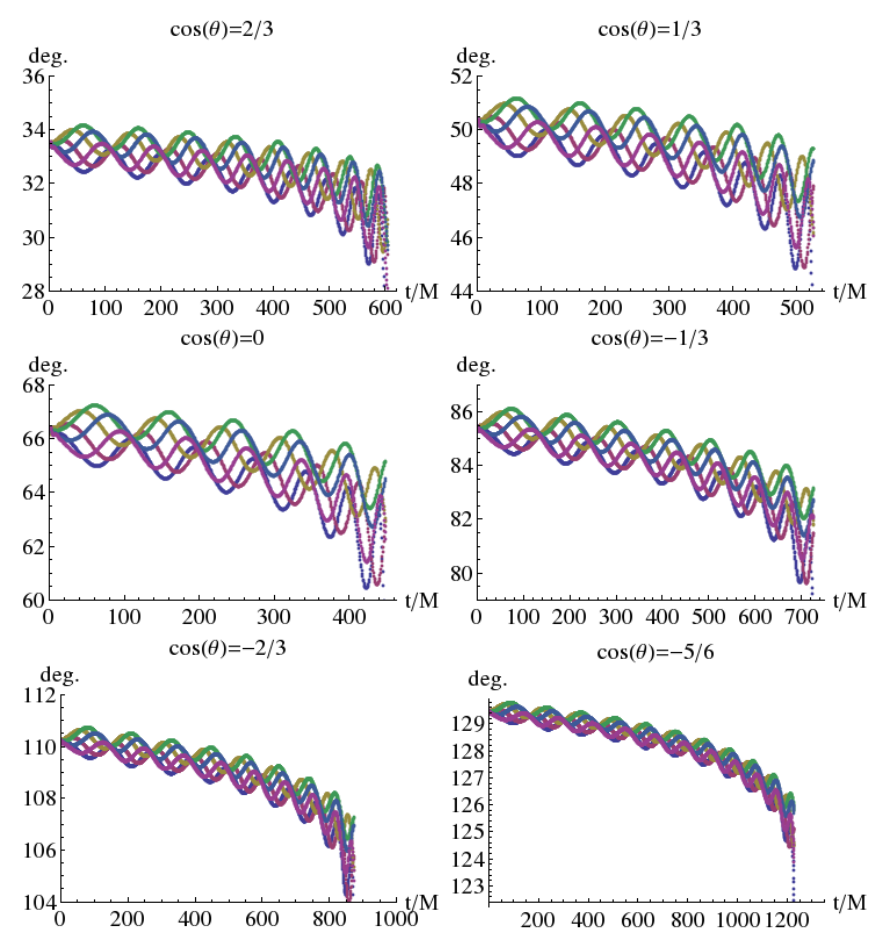

FIG. 6: The angle between $\hat{J}$ and $\hat{S}_{1}=\hat{S}_{2}$ for the SPTH48 (top left), SPTH70 (top right), SPTH90 (middle left), SPTH110 (middle right), SPTH132 (bottom left), and SPTH146 (bottom right) configurations.

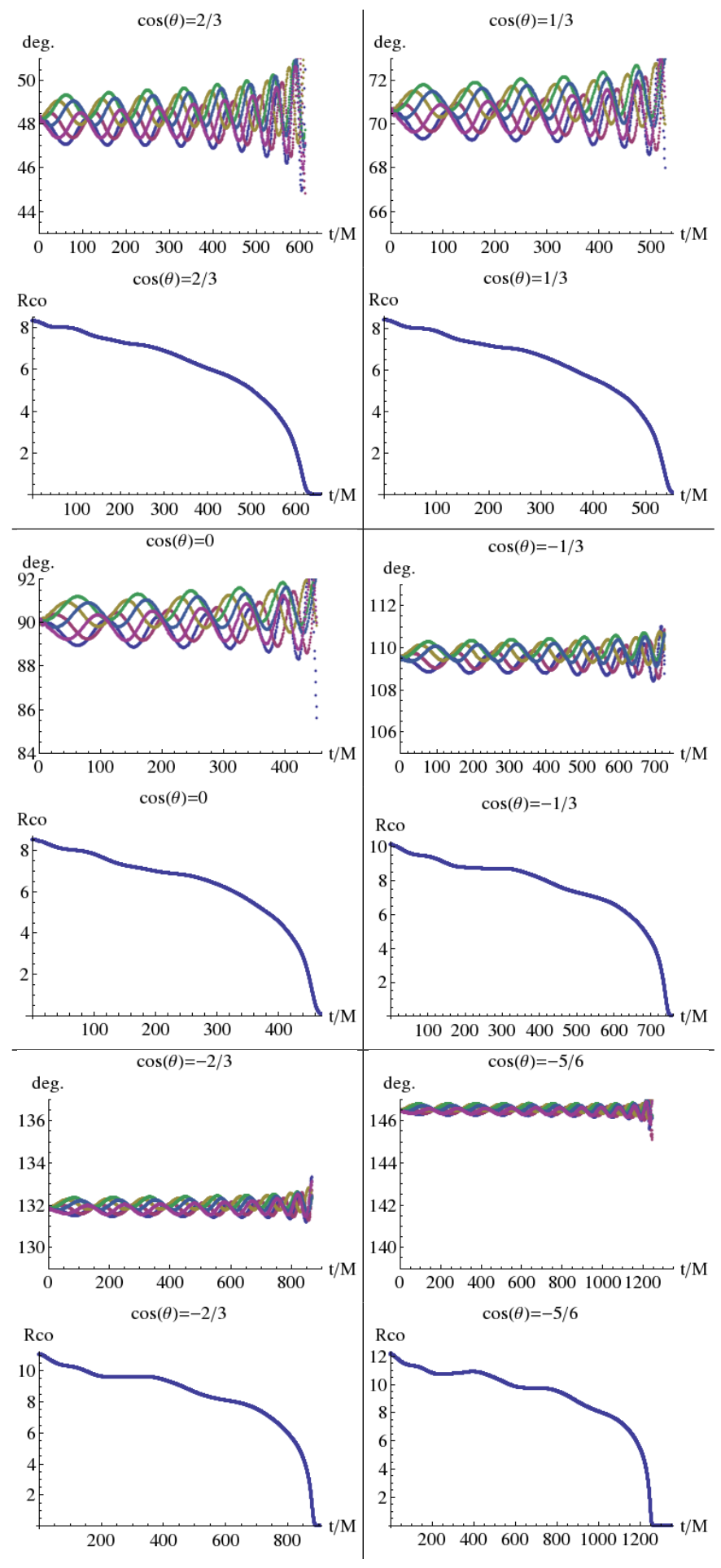

FIG. 7: The angle between $\hat{L}(t)$ (radiation) and $\hat{S}_{1}=\hat{S}_{2}$ for the SPTH48 (top left), SPTH70 (top right), SPTH90 (middle left), SPTH110 (middle right), SPTH132 (bottom left), and SPTH146 (bottom right) configurations. The lower plots show the orbital separation versus the same time scale. 

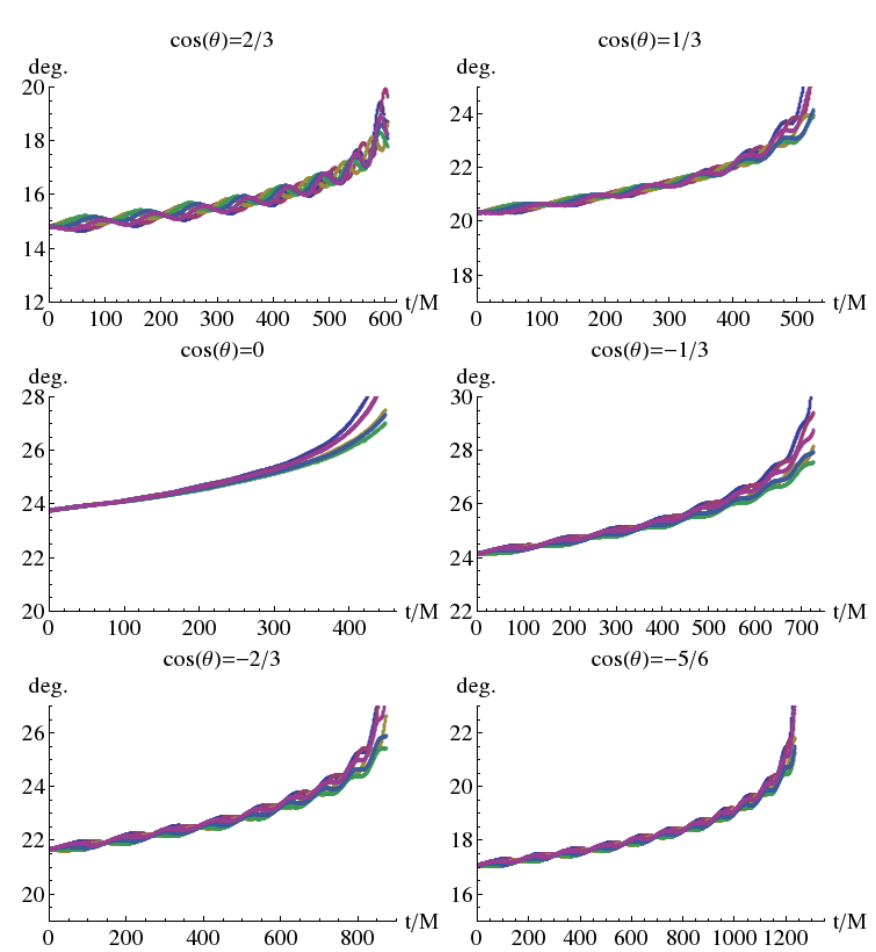

FIG. 8: The angle between $\hat{J}$ and $\hat{L}(t)$ for the SPTH48 (top left), SPTH70 (top right), SPTH90 (middle left), SPTH110 (middle right), SPTH132 (bottom left), and SPTH146 (bottom right) configurations.

Given that we see an approximate conservation of the magnitude of the total spin $\vec{S}$ (see Fig. 5), as well as the conservation (see Fig. 7) of the component $\vec{S}$ along the angular momentum $\vec{L}$ (denoted by $S_{\|}=\vec{S} \cdot \hat{L}$ ) we can deduce that the angle between $\vec{J}$ and $\vec{L}$ should increase on secular timescales, while the angle between $\vec{J}$ and $\vec{S}$ should decrease. The argument is as follows; since $|\vec{S}|^{2}=$ $S_{\perp}^{2}+S_{\|}^{2}$ and both $|\vec{S}|$ and $S_{\|}$are conserved, then $S_{\perp}$ must be conserved, as well (in magnitude only). $|\vec{J}|$, on the other hand decreases on secular timescales due to the radiation of angular momentum to infinity. Hence (see Fig. 9

$$
\cos ^{2} \gamma=(\hat{J} \cdot \hat{L})^{2}=1-\frac{S_{\perp}^{2}}{J^{2}}
$$

decreases on the same secular timescale. For the configurations considered here, $\gamma<\pi / 2$ (i.e. there is only simple precession), hence $\gamma$ increases (as seen in Fig. 8).

Likewise we can show that the constancy of the spin magnitude and its projection along $\vec{L}$ leads to the decrease of the angle between $\vec{J}$ and $\vec{S}$ seen in Fig. 6. Again, from $\vec{J}=\vec{L}+\vec{S}$, we have (see Fig. 9 )

$$
\cos ^{2}(\theta-\gamma)=(\hat{J} \cdot \hat{S})^{2}=1-\frac{L^{2}}{J^{2}} \sin ^{2} \theta
$$

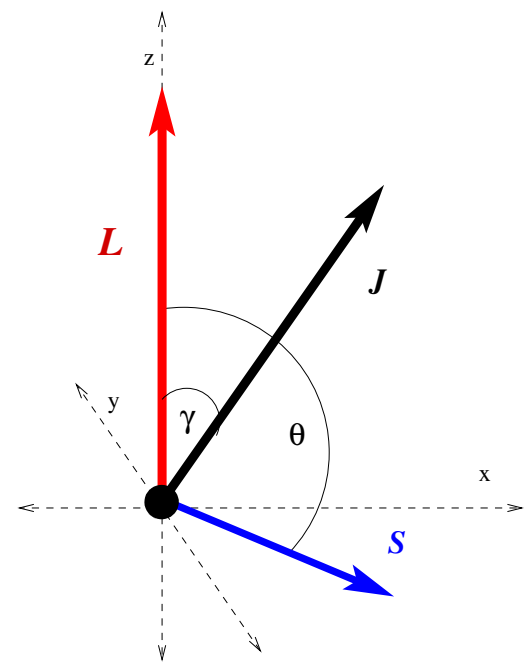

FIG. 9: The orbital, spin, and net angular momentum. We choose the polar axis to always lie along the orbital angular momentum. The angles $\gamma$ and $\theta$ measure the inclination angles of $\vec{J}$ and $\vec{S}$, respectively.

and since

$$
\frac{J^{2}}{L^{2}}=1+\frac{2}{L}(\hat{L} \cdot \vec{S})+\frac{S^{2}}{L^{2}}
$$

will increase or decrease with the radiation of $L$ depending on the sign of $(2 \vec{L}+\vec{S}) \cdot \vec{S}$ (and hence depending on the sign of $(\hat{L} \cdot \vec{S})$ for the simple precession case). Thus for our configurations, the angle $(\theta-\gamma)$ has to decrease on the secular scale of the orbital decay (as seen in Fig. 66).

We now briefly return to the question of which approximation to the direction of the orbital angular momentum provides the best properties for subsequent modeling of BHBs. In Fig. 10 and 11 we plot the directions defined by O'Shaughnessy et al., $\hat{O}_{\psi}, \hat{O}_{N}, \hat{O}_{h}$, the directions defined by Boyle, $\hat{B}_{h}(t), \hat{B}_{N}(t)$, as well as $\hat{L}(t)$, and the purely coordinate-based $\hat{L}_{\text {coord }}(t)$. Based on these figures, one may suspect that $\hat{L}_{\text {coord }}(t)$ most faithfully follows the dynamics of the binary (i.e. nutation effects are present). However, for our purposes, we are interested in determining the direction $\hat{V}$ such that $\hat{V} \cdot \hat{S}$ is as constant as possible.

To this end, we compare the time dependence of the two angles $\{\vec{S}(t), \vec{L}(t)\}$ and $\left\{\vec{S}(t), \vec{L}_{\text {coord }}(t)\right\}$. As shown in Fig. 12 in all cases we saw that $\{\vec{S}(t), \vec{L}(t)\}$ shows a smaller secular change than $\left\{\vec{S}(t), \vec{L}_{\text {coord }}(t)\right\}$ (the effect was not always significant). This raises the question of whether the more geometric directions of O'Shaughnessy et al. and Boyle lead to even better performance. In Fig. 13. we plot the angles $\left\{\vec{S}(t), \vec{O}_{N}(t)\right\},\left\{\vec{S}(t), \vec{O}_{h}(t)\right\}$, $\left\{\vec{S}(t), \vec{B}_{N}(t)\right\},\left\{\vec{S}(t), \vec{B}_{h}(t)\right\}$, and $\{\vec{S}(t), \vec{L}(t)\}$. Here we find that the angle between $\vec{S}(t)$ and $\vec{L}(t)$ is conserved to a much higher degree than any of the other angles. 

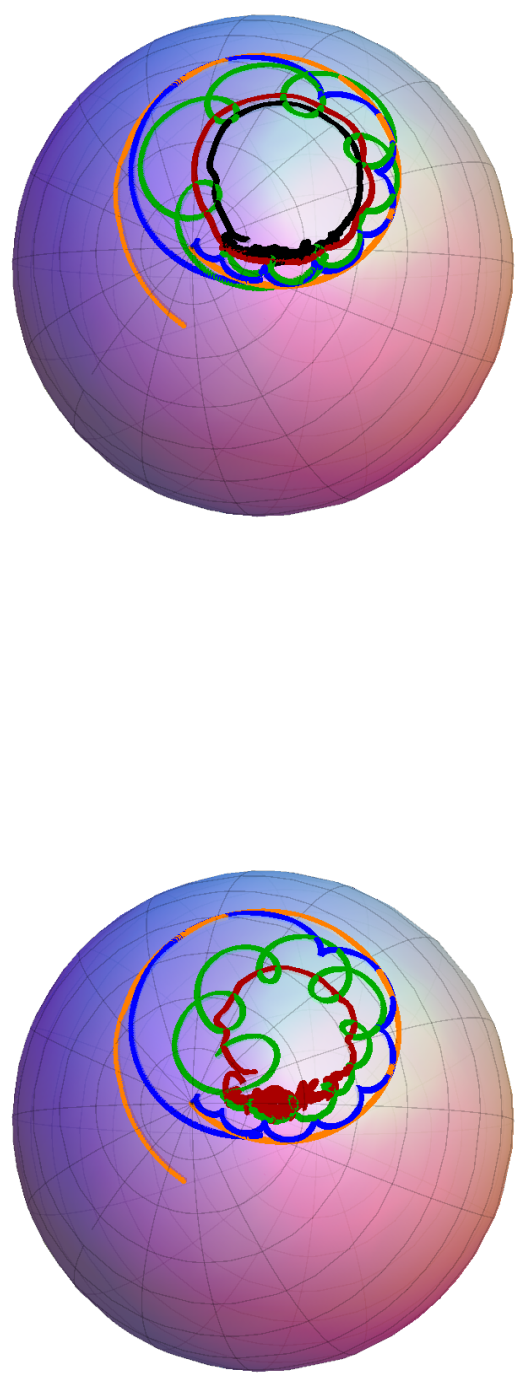

FIG. 10: A comparison of the directions of $\hat{L}_{\text {coord }}$ (blue), $\hat{L}_{\text {rad }}$ (orange), with the principle eigenvector direction of $\langle\mathbf{L L}\rangle$ (see Ref. [56]) defined using $\psi_{4}$ (black), $N$ (red), and $h$ (green) (top), and with the vectors $\langle\mathbf{L L}\rangle^{-1}\langle\mathbf{L T}\rangle$ (see Ref. [57]) defined using $N$ (red) and $h$ (green) (bottom). Both plots are from the SPTH90PH0 configuration.

\section{B. Results from the UD configurations}

In order to model the dependence of the final mass (or radiated energy) and spin of the merger remnant of BHBs as a function $\Delta_{\|}$, we perform a series of additional runs of equal-mass binaries with one $\mathrm{BH}$ spin aligned and the

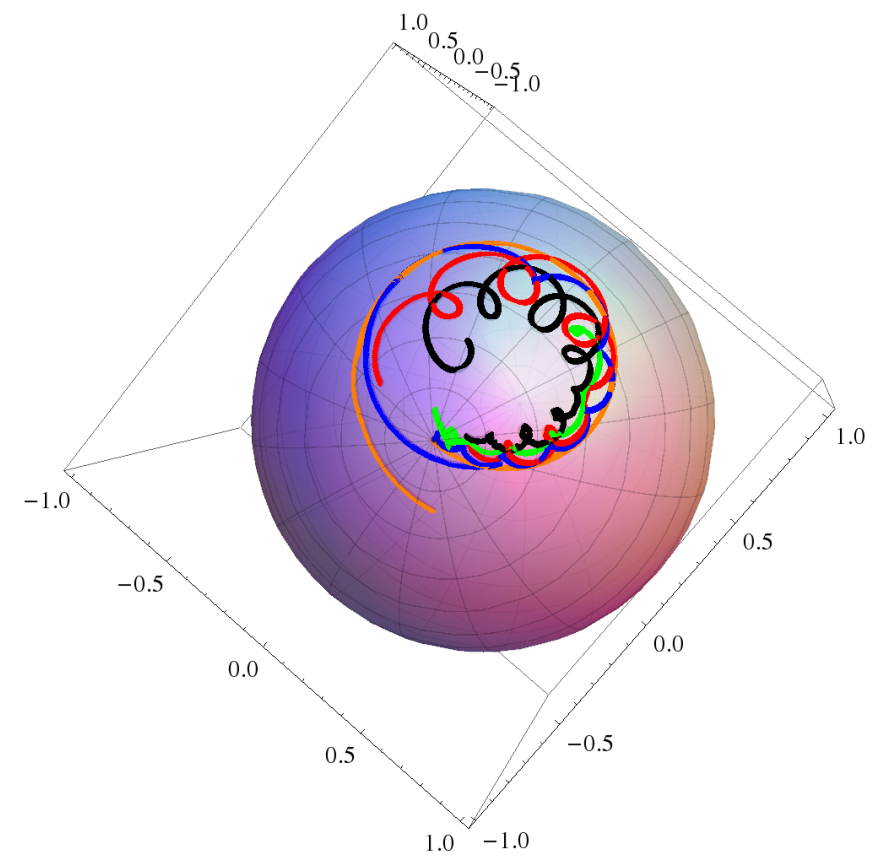

FIG. 11: A plot comparing different measures of the direction of orbital angular momentum for the SPTH90PH0 configuration. Plotted are the trajectories of $\hat{B}_{h}(t)$ (black), $\hat{O}_{h}(t)$ (red), $\overrightarrow{\delta J}_{\text {rad }}$ (green), $\hat{L}_{\text {coord }}$ (blue), and $\hat{L}(t)$ (orange). $\hat{L}_{\text {coord }}$ shows interesting nutation behavior not seen in the other curves.

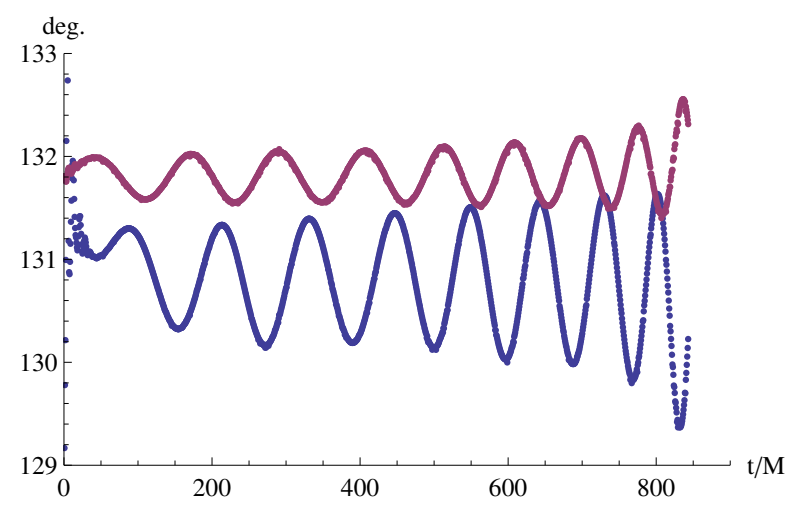

FIG. 12: A plot comparing the angles $\{\vec{S}(t), \vec{L}(t)\}$ (upper, red) and $\left\{\vec{S}(t), \vec{L}_{\text {coord }}(t)\right\}$ (lower, blue) for the SPTH132PH0 configuration. Note how $\left\{\vec{S}(t), \vec{L}_{\text {coord }}(t)\right\}$ shows a stronger secular trend.

other counteraligned to the orbital angular momentum. These up-down (UD) configurations are non precessing. For these configurations, we are interested in calculating the final remnant properties to high accuracy. As shown in Fig. 14 the calculation of the total radiated mass and the final remnant spin require increasingly higher resolutions as the initial spin magnitude increases.

Since the UD configuration with initial spins $\pm \alpha$ is equivalent to the configuration with initial spins $\mp \alpha$, the remnant spin and total mass cannot depend on the sign of $\alpha$ (and hence cannot depend on the sign of $\Delta_{\|}$). Con- 


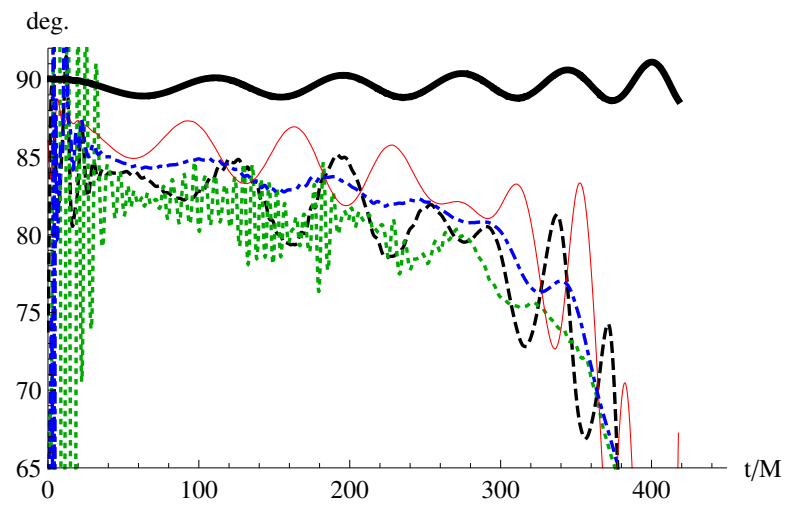

FIG. 13: The angle between $\vec{S}(t)$ and $\vec{B}_{h}(t)$ (black, dashed), $\vec{B}_{n}(t)$ (green, dotted), $\vec{O}_{h}(t)$ (red, solid), $\vec{O}_{n}(t)$ (blue, dasheddot), and $\vec{L}(t)$ (black, solid) for the SPTH90PH0 configuration. All angles show a strong secular trend except the angle between $\vec{S}(t)$ and $\vec{L}(t)$.
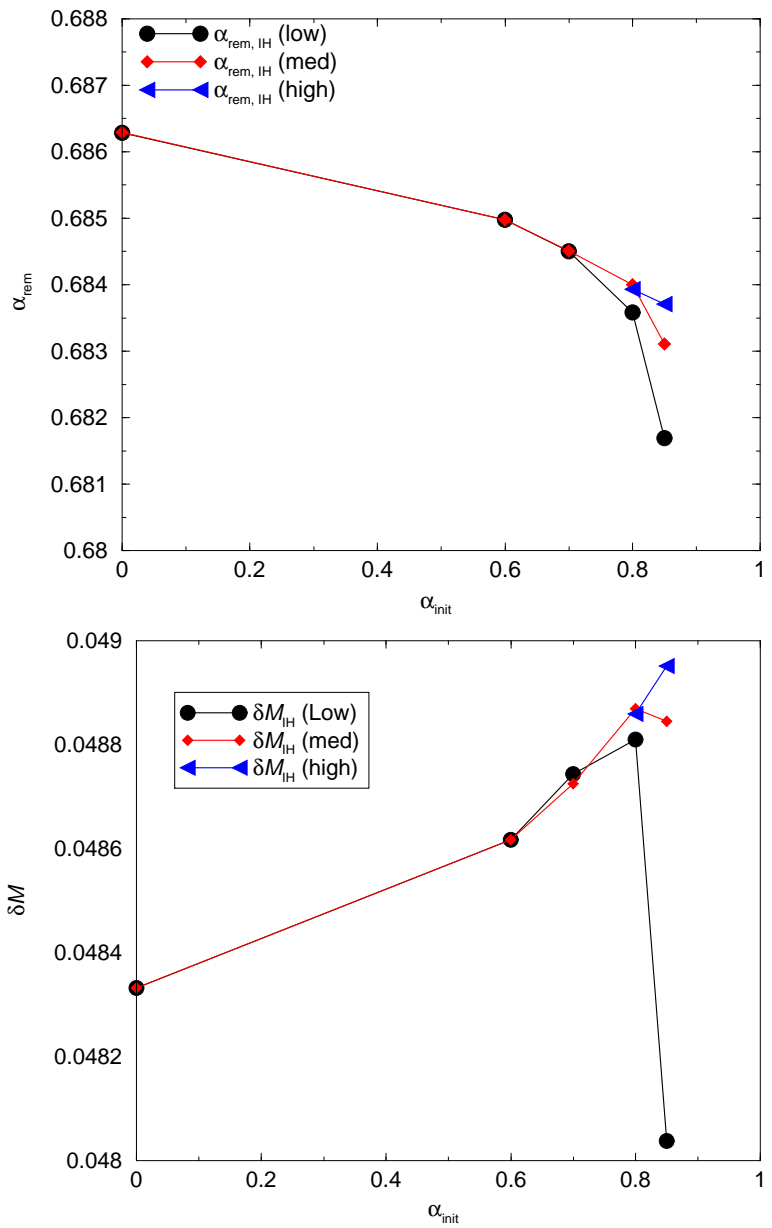

FIG. 14: The total radiated energy $(\delta \mathcal{M})$ and final remnant spin $\alpha_{\text {rem }}$ versus initial spin for the UD configurations. Note how trends for both the radiated mass and final remnant spins need higher resolution runs at high spins.
TABLE II: The values for the parameters in Eqs. 10 and (11) after fitting to the results of the UD configurations. Here " 0 " indicates that the uncertainty in the remaining fitting parameters was substantially reduced when this coefficient was set to zero.

\begin{tabular}{ll|ll}
\hline \hline$A_{0}$ & $(0.470978 \pm 0.000051)$ & $E_{0}$ & $(0.048332 \pm 0.000002)$ \\
$A_{2}$ & $-(0.00492 \pm 0.00010)$ & $E_{2}$ & $(0.000743 \pm 0.000018)$ \\
$A_{4}$ & 0 & $E_{4}$ & $(0.000124 \pm 0.000028)$ \\
\hline \hline
\end{tabular}

sequently, we fit the remnant $\operatorname{spin} \alpha_{\text {rem }}$ and total radiated mass to the forms

$$
\begin{gathered}
\alpha_{\mathrm{rem}}^{2}=A_{0}+A_{2}\left(\frac{\Delta_{\|}}{m^{2}}\right)^{2}+A_{4}\left(\frac{\Delta_{\|}}{m^{2}}\right)^{4}, \\
\delta \mathcal{M}=E_{0}+E_{2}\left(\frac{\Delta_{\|}}{m^{2}}\right)^{2}+E_{4} \cdot\left(\frac{\Delta_{\|}}{m^{2}}\right)^{4},
\end{gathered}
$$

We give the results from the fits in Table II

\section{MODELING PRECESSING BINARIES AND THEIR REMNANTS}

In this section we use analytic techniques to gain insight into the observed dynamics of precessing BHBs and the modeling of the merger remnant.

\section{A. Post-Newtonian Analysis}

As show in Fig. 7, the angle between $\vec{L}$ and $\vec{S}$ oscillates at roughly the orbital frequency, and the amplitude of these oscillations increases as the binary inspirals. In addition, the baseline of these oscillations is a function of the azimuthal angle $\phi$. This behavior is predicted by PN theory, as we will demonstrate here.

At $2 \mathrm{PN}$ order, the evolution of the total spin for equalmass binaries is given by [59]

$$
\frac{d \vec{S}}{d t}=\frac{7}{2 r^{3}}(\vec{L} \times \vec{S})+\frac{3}{r^{3}}(\hat{n} \cdot \vec{S})(\hat{n} \times \vec{S}),
$$

where $\vec{L}$ is the orbital angular momentum and $\hat{n}$ is the unit vector pointing along the line joining the two BHs. At this order, the evolution is conservative, and hence $\dot{\vec{J}}=\dot{\vec{L}}+\dot{\vec{S}}=0$, where an overdot means total time derivative. Consequently, $\vec{S} \cdot \dot{\vec{S}}=0$ and therefore

$$
\frac{d(\vec{J} \cdot \vec{S})}{d t}=\frac{d(\vec{L} \cdot \vec{S})}{d t}=-\frac{d(\vec{J} \cdot \vec{L})}{d t}=\vec{L} \cdot \dot{\vec{S}} .
$$

While the magnitude of $\vec{S}$ remains constant the magnitude of $\vec{L}$ varies according to

$$
\frac{d(\vec{S} \cdot \vec{S})}{d t}=0, \quad \frac{d(\vec{L} \cdot \vec{L})}{d t}=-2 \vec{L} \cdot \dot{\vec{S}} .
$$


The variation of the component of $\vec{S}$ along $\vec{L}$ is then given by

$$
\begin{aligned}
\frac{d}{d t}(\hat{L} \cdot \vec{S}) & =\hat{L} \cdot \dot{\vec{S}}+\dot{\hat{L}} \cdot \vec{S} \\
& =\frac{3}{r^{3}}(\hat{n} \cdot \vec{S})(\hat{\lambda} \cdot \vec{S})[1+(\hat{L} \cdot \vec{S}) / \ell] \\
=\dot{S}_{\hat{L}} & =\frac{3}{r^{3}}\left(S_{\hat{n}}\right)\left(S_{\hat{\lambda}}\right)\left[1+\left(S_{\hat{L}}\right) / \ell\right]
\end{aligned}
$$

where $\hat{n}$ and $\hat{\lambda}$ are the unit vectors on the orbital plane along the radial and tangential directions, and $\hat{L}=\hat{n} \times \hat{\lambda}$ and $\ell=\|\vec{L}\|$.

Similarly, the variation of the component of $\vec{S}$ perpendicular to $\vec{L}$ is given by

$$
\begin{aligned}
\frac{d}{d t}(\hat{L} \times \vec{S}) & =\dot{S}_{\hat{n}} \hat{\lambda}-\dot{S}_{\hat{\lambda}} \hat{n}+S_{\hat{n}} \dot{\hat{\lambda}}-S_{\hat{\lambda}} \dot{\hat{n}} \\
& =\hat{L} \times \dot{\vec{S}}+\dot{\hat{L}} \times \vec{S}
\end{aligned}
$$

where

$$
\begin{aligned}
& \dot{\hat{L}}=\left(-\frac{7}{2}+\frac{3 S_{\hat{L}}}{\ell}\right) \frac{S_{\hat{n}}}{r^{3}} \hat{\lambda}+\frac{7 S_{\hat{\lambda}}}{2 r^{3}} \hat{n}, \\
& \dot{\hat{\lambda}}=-\left(-\frac{7}{2}+\frac{3 S_{\hat{L}}}{\ell}\right) \frac{S_{\hat{n}}}{r^{3}} \hat{L}-\frac{V_{\lambda}}{r} \hat{n} \\
& \dot{\hat{n}}=\frac{V_{\lambda}}{r} \hat{\lambda}-\frac{7 S_{\hat{\lambda}}}{2 r^{3}} \hat{L}
\end{aligned}
$$

where $V_{\lambda}$ is the tangential velocity of the binary.

The two transverse components of the total spin therefore obey

$$
\begin{aligned}
\dot{S}_{\hat{n}} & =\Omega_{-} S_{\hat{\lambda}}, \\
-\dot{S}_{\hat{\lambda}} & =\Omega_{+} S_{\hat{n}},
\end{aligned}
$$

where

$$
\Omega_{-}=\frac{V_{\lambda}}{r}-\frac{7 \ell}{2 r^{3}}\left(1+S_{\hat{L}} / \ell\right)
$$

and

$$
\Omega_{+}=\Omega_{-}+\frac{3 S_{\hat{L}}}{r^{3}}\left(1+S_{\hat{L}} / \ell\right) .
$$

Equations (15) and (18) represent a system of equations for the evolution of the precessing spin which can solved in the small oscillations regime to give

$$
\begin{aligned}
& S_{\hat{n}}(t)=S_{\hat{n}}(0) \cos \left(\Omega_{ \pm} t\right)+\frac{\Omega_{-}}{\Omega_{ \pm}} S_{\hat{\lambda}}(0) \sin \left(\Omega_{ \pm} t\right) \\
& S_{\hat{\lambda}}(t)=-\frac{\Omega_{ \pm}}{\Omega_{-}} S_{\hat{n}}(0) \sin \left(\Omega_{ \pm} t\right)+S_{\hat{\lambda}}(0) \cos \left(\Omega_{ \pm} t\right) \\
& S_{\hat{L}}(t)=S_{\hat{L}}(0)+\frac{3}{2 \Omega_{-} r^{3}}\left[S_{\hat{n}}^{2}(t)-S_{\hat{n}}^{2}(0)\right]
\end{aligned}
$$

where $\Omega_{ \pm}^{2}=\Omega_{+} \Omega_{-}$,

$$
S_{\hat{n}}(0)=S \sin \theta_{0} \cos \phi_{0},
$$

$$
S_{\hat{\lambda}}(0)=-S \sin \theta_{0} \sin \phi_{0},
$$

and the angles $\phi_{0}$ and $\theta_{0}$ give the initial azimuthal and polar orientations of $\vec{S}$ (where the polar axis is aligned with $\hat{L}$ ). These PN expressions thus reproduce the amplitude and frequency of the oscillations observed in Fig. 7. Taking the orbit average we get,

$$
\begin{aligned}
& \left\langle S_{\hat{L}}(t)\right\rangle=S_{\hat{L}}(0)+ \\
& \frac{3|\vec{S}|^{2}}{4 r^{3} \Omega_{ \pm}^{2}} \sin ^{2}\left(\theta_{0}\right)\left[\Omega_{-} \cos ^{2}\left(\phi_{0}\right)-\Omega_{+} \sin ^{2}\left(\phi_{0}\right)\right] .
\end{aligned}
$$

Since $S$ is conserved, we find that the orbit-averaged angle $\cos ^{-1}(\hat{S} \cdot \hat{L})$ changes by less than three degrees from infinite separation down to separations where the orbital frequency approaches $0.1 / \mathrm{M}$ (where the PN approximation breaks down) and that the average angle depends of $\phi_{0}$ (i.e. the initial azimuthal orientation).

\section{B. The Hangup effect in precessing binaries}

To estimate which configuration maximizes the amount of angular momentum radiated perpendicular to $\vec{J}$, we look for configurations that maximize the angle $\gamma$ between the orbital angular momentum $\vec{L}$ and $\vec{J}$ near merger (at merger, there is an intense burst of angular momentum radiated).

Since $\vec{J}=\vec{L}+\vec{S}, \cos \gamma$ is given by

$$
\cos \gamma=\frac{\vec{L} \cdot \vec{J}}{|\vec{L}||\vec{J}|}=\frac{1+(S / L) \cos \theta}{\sqrt{1+2(S / L) \cos \theta+(S / L)^{2}}},
$$

where $\theta$ is the angle between $\vec{S}$ and $\vec{L}$ (see Fig. 9).

While $S$ is preserved during evolutions of our configurations (see Fig. 5), to estimate $L$ at merger we use the hangup configuration results (as this proves to be the dominant effect for precessing binaries [21]). Our ansatz is

$$
L^{\text {hangup }}=J_{\text {rem }}^{\text {hangup }}-S \cos \theta
$$

where $L_{\text {merger }}=L^{\text {hangup }}$ and $J_{\text {rem }}^{\text {hangup }}$ is given by 60 .

$$
\begin{aligned}
J_{\text {rem }}^{\text {hangup }} / M_{\text {rem }}^{2}= & 0.686402+0.30660 s-0.02684 s^{2} \\
& -0.00980 s^{3}-0.00499 s^{4}, \\
M_{\text {rem }} / M_{\text {init }}= & \left(1-0.00258+\frac{0.07730}{s-1.6939}\right),
\end{aligned}
$$

$M_{\text {init }}=M_{1}+M_{2}$ (the horizons masses $M_{1}$ and $M_{2}$ are equal in this case), $s=2 S / M_{\text {init }}^{2} \cos \theta$ is the component of the dimensionless spin along the orbital angular momentum (note and $s=0.8 \cos \theta$ and $S / M_{\mathrm{rem}}^{2}=0.4 M^{2} / M_{\mathrm{rem}}^{2}$ for our configurations). By minimizing $\cos \gamma$ with respect to $\cos \theta$, we find that

$$
\gamma \approx 34^{\circ} \text { and } \theta \approx 110^{\circ}
$$


Extrapolating the above expressions to a pair of maximally spinning black holes gives,

$$
\gamma_{\max } \approx 42^{\circ} \text { and } \theta_{\max } \approx 117^{\circ} \text {. }
$$

Thus we find by using straightforward geometric arguments that the largest precession of the total angular momentum will occur with spins partially anti-aligned with the orbital angular momentum, which agrees qualitatively with our results in Fig. 4 .

\section{Symmetries}

In this section, we use $\vec{J}$ to denote the final remnant spin angular momentum and $\vec{S}$ to denote the spin of the precursor binary. Here, the component $V_{\perp}$ of any vector $\vec{V}$ is understood to be $V_{\perp}=\vec{V} \cdot \hat{n}$, where $\hat{n}$ is a vector in the orbital plane (e.g. the direction from $\mathrm{BH} 1$ to $\mathrm{BH} 2$ ). We always define these directions such that, under exchange of labels $\mathrm{BH} 1 \leftrightarrow \mathrm{BH} 2, \hat{n}$ changes sign.

In order to develop a new phenomenological formula for the final masses and spins of merged binary black holes, we consider a Taylor expansion of those quantities [25, 26] in terms of spin components $S_{\|}, S_{\perp}, \Delta_{\|}, \Delta_{\perp}$. This choice of variables allows us to relate the expansion of the final mass (or energy radiated) and the final spin with the expansion we developed for modeling the final recoil velocity in [17. There we argued that the leadingorder dependence of the recoil could be modeled by the variable $\vec{\Delta}$ rather than the alternative $\overrightarrow{\delta S}=\vec{S}_{2}-\vec{S}_{1}$ (see Fig. 2 in Ref. [17]). In the equal-mass limit, $\vec{\Delta}=2 \overrightarrow{\delta S}$ and the two variables are equivalent. However, for $q<1$, we showed that the spin-dependence of the recoil is fit with fewer terms if we expand in terms of $\vec{\Delta}$. Here too, we may consider alternative variables to $\vec{S}$, for example, $\vec{S}_{0}=m\left(\vec{S}_{2} / m_{2}+\vec{S}_{1} / m_{1}\right)$ or the $\vec{S}_{\text {eff }}=\vec{S} / 4+3 \vec{S}_{0} / 4$ which arise from the leading-order spin terms in the PN Hamiltonian 61]. While the infinite series expansion for all of these choices of spin variables are equivalent, a low-order truncated series in a preferred set of variables may have a substantially smaller error than the other choices. Determining the best choice of the variables will involve a detailed analysis of a large set of new unequal mass binaries that we will postpone for a forthcoming paper 62 . Note that the alternative variables we considered share the symmetries of $\vec{S}$, hence the expansion we give below can be easily adapted to them.

A Taylor expansion of a function with $v$ independent variables of a given order of expansion $o$ has $n$ terms, where $n$ is given by 63 .

$$
n=\frac{(o+v-1) !}{o !(v-1) !} .
$$

Our models will depend on five variables, $\delta m, S_{\|}, S_{\perp}$,
$\Delta_{\|}$, and $\Delta_{\perp}$. Hence $n=1,5,15,35,70, \cdots$ for $0^{\text {th }}$ order through $4^{\text {th }}$ order expansions, respectively. However,

TABLE III: The number of terms at a given order of expansion (with respect to $\vec{S}$ or $\vec{\Delta}$ ) for $J_{\|}$and $J_{\perp}$ and a subtotal including all terms in both $J_{\|}$and $J_{\perp}$ up to the given order. Note that the subtotals agree with formula 33 indicating that all possible terms are used in expanding $J_{\|}$and $J_{\perp}$. Here 1 indicates terms that are retained in the equal-mass limit (or proportional to even powers of $\delta m$ ) and $\delta m$ indicates terms proportional to $\delta m$ (and other odd powers). The number of terms in an expansion of $M_{\text {rem }}$ agrees with the corresponding number of terms in $J_{\|}$and is provided for convenience.

\begin{tabular}{|c|c|c|c|c|c|c|c|c|c|c|c|}
\hline \hline Order & 0th & 0th & 1st & 1st & 2nd & 2nd & 3rd & 3rd & 4th & 4th & total \\
\hline term & 1 & $\delta m$ & 1 & $\delta m$ & 1 & $\delta m$ & 1 & $\delta m$ & 1 & $\delta m$ & Sum \\
\hline \hline$J_{\|}$ & 1 & 0 & 1 & 1 & 4 & 2 & 5 & 5 & 11 & 8 & 38 \\
\hline$J_{\perp}$ & 0 & 0 & 1 & 1 & 2 & 2 & 5 & 5 & 8 & 8 & 32 \\
\hline subtotal & - & 1 & - & 5 & - & 15 & - & 35 & - & 70 & - \\
\hline \hline$M_{\text {rem }}$ & 1 & 0 & 1 & 1 & 4 & 2 & 5 & 5 & 11 & 8 & 38 \\
\hline \hline
\end{tabular}

TABLE IV: Symmetry properties of key quantities under parity $(\mathrm{P})$ and exchange of labels $(\mathrm{X})$ [i.e., $\mathrm{BH} 1 \leftrightarrow \mathrm{BH} 2]$.

\begin{tabular}{|l|c|c|}
\hline \hline Symmetry & $\mathrm{P}$ & $\mathrm{X}$ \\
\hline \hline$J_{\|} / m^{2}$ & + & + \\
\hline$J_{\perp} / m^{2}$ & - & - \\
\hline$m / M$ & + & + \\
\hline$S_{\perp} / M^{2}=\left(S_{1}+S_{2}\right)_{\perp} / M^{2}$ & - & - \\
\hline$S_{\|} / M^{2}=\left(S_{1}+S_{2}\right)_{\|} / M^{2}$ & + & + \\
\hline$\Delta_{\perp} / M^{2}=\left(S_{2} / m_{2}-S_{1} / m_{1}\right)_{\perp} / M$ & - & + \\
\hline$\Delta_{\|} / M^{2}=\left(S_{2} / m_{2}-S_{1} / m_{1}\right)_{\|} / M$ & + & - \\
\hline$\hat{n}=\hat{r}_{1}-\hat{r}_{2}$ & + & - \\
\hline$\delta m=\left(m_{1}-m_{2}\right) / M$ & + & - \\
\hline \hline
\end{tabular}

only certain combinations of variables are allowed according to the symmetry properties of the object we want to build up. In order to take into account the correct combinations of variables for each component of the final mass and spin of the remnant black hole at a given order, we consider the symmetry properties summarized in Table IV (note that this corrects typos in Table I of Ref [17]). While the number of terms in $J_{\|}$and $J_{\perp}$ is less than Eq. (33), the sum of the terms in both agrees with Eq. (33), as seen in Table III. This is due to the fact that an arbitrary product of powers of $S_{\|}, S_{\perp}, \Delta_{\|}$, and $\Delta_{\perp}$ either has the has the symmetries of $J_{\|}$or $J_{\perp}$, or this product times $\delta m$ has the symmetries of $J_{\|}$or $J_{\perp}$.

The possible terms to a given expansion order in spin (i.e., products of $S$ and $\Delta$ ) are summarized in Tables V. VI. In our phenomenological description the terms in Tables V and VI are all multiplied by fitting coefficients. Note that the coefficients of these terms can depend on higher powers of $\delta m$ (i.e., higher even powers in $\delta m$ for those terms proportional to $\delta m^{0}$, and odd powers for terms proportional to $\delta m$ ). 
TABLE V: Parameter dependence at each order of expansion for the final spin component perpendicular to the reference $\vec{L}$ direction. Here 1 indicates terms present in the equal-mass limit (or proportional to even powers of $\delta m$ ) and $\delta m$ indicates terms proportional to $\delta m$ (and other odd powers).

\begin{tabular}{|c|l|}
\hline \hline$J_{\perp}$ & Oth order \\
\hline 1 & 0 \\
\hline$\delta m$ & 0 \\
\hline \hline$J_{\perp}$ & 1 st order \\
\hline 1 & $S_{\perp}$ \\
\hline$\delta m$ & $\Delta_{\perp}$ \\
\hline \hline$J_{\perp}$ & 2nd order \\
\hline 1 & $S_{\perp} \cdot S_{\|}+\Delta_{\|} \cdot \Delta_{\perp}$ \\
\hline$\delta m$ & $S_{\perp} \cdot \Delta_{\|}+\Delta_{\perp} \cdot S_{\|}$ \\
\hline \hline$J_{\perp}$ & $3 \mathrm{rd}$ order \\
\hline 1 & $\Delta_{\|} \cdot \Delta_{\perp} \cdot S_{\|}+S_{\perp} \cdot S_{\|}^{2}+S_{\perp} \cdot \Delta_{\|}^{2}+S_{\perp}^{3}+S_{\perp} \cdot \Delta_{\perp}^{2}$ \\
\hline$\delta m$ & $\Delta_{\perp} \cdot \Delta_{\|}^{2}+\Delta_{\perp} \cdot S_{\|}^{2}+S_{\perp} \cdot \Delta_{\|} \cdot S_{\|}+\Delta_{\perp} \cdot S_{\perp}^{2}+\Delta_{\perp}^{3}$ \\
\hline \hline$J_{\perp}$ & $4 \operatorname{th}$ order \\
\hline 1 & $\Delta_{\perp} \cdot \Delta_{\|}^{3}+S_{\perp} \cdot S_{\|}^{3}+S_{\perp} \cdot S_{\|} \cdot \Delta_{\|}^{2}+\Delta_{\perp} \cdot \Delta_{\|} \cdot S_{\|}^{2}+S_{\perp}^{3} \cdot S_{\|}+\Delta_{\perp}^{3} \cdot \Delta_{\|}+S_{\perp}^{2} \cdot \Delta_{\perp} \cdot \Delta_{\|}+S_{\perp} \cdot \Delta_{\perp}^{2} \cdot S_{\|}$ \\
\hline$\delta m$ & $S_{\perp} \cdot \Delta_{\|}^{3}+\Delta_{\perp} \cdot S_{\|}^{3}+\Delta_{\perp} \cdot S_{\|} \cdot \Delta_{\|}^{2}+S_{\perp} \cdot \Delta_{\|} \cdot S_{\|}^{2}+S_{\perp}^{3} \cdot \Delta_{\|}+\Delta_{\perp}^{3} \cdot S_{\|}+S_{\perp}^{2} \cdot \Delta_{\perp} \cdot S_{\|}+\Delta_{\perp}^{2} \cdot S_{\perp} \cdot \Delta_{\|}$ \\
\hline \hline
\end{tabular}

TABLE VI: Parameter dependence at each order of expansion for the final spin component along the reference $\vec{L}$ direction and similarly for the remnant mass $M_{\text {rem}}$. Here 1 indicates terms present in the equal-mass limit (or proportional to even powers of $\delta m)$ and $\delta m$ indicates terms proportional to $\delta m$ (and to other odd powers).

\begin{tabular}{|c|c|}
\hline$J_{\|}$or $M_{\text {rem }}$ & 0th order \\
\hline 1 & $L(S=0)$ or $M(S=0)$ \\
\hline$\delta m$ & 0 \\
\hline$J_{\|}$or $M_{\mathrm{rem}}$ & 1st order \\
\hline 1 & $S_{\|}$ \\
\hline$\delta m$ & $\Delta_{\|}$ \\
\hline$J_{\|}$or $M_{\text {rem }}$ & 2nd order \\
\hline 1 & $\Delta_{\|}^{2}+S_{\|}^{2}+\Delta_{\perp}^{2}+S_{\perp}^{2}$ \\
\hline$\delta m$ & $\Delta_{\|} \cdot S_{\|}+\Delta_{\perp .} S_{\perp}$ \\
\hline$J_{\|}$or $M_{\text {rem }}$ & 3rd order \\
\hline 1 & $S_{\|} \cdot \Delta_{\|}^{2}+S_{\|} \cdot S_{\perp}^{2}+\Delta_{\perp} \cdot S_{\perp} \cdot \Delta_{\|}+S_{\|} \cdot \Delta_{\perp}^{2}+S_{\|}^{3}$ \\
\hline$\delta m$ & $\Delta_{\perp} \cdot S_{\perp} \cdot S_{\|}+\Delta_{\|} \cdot S_{\|}^{2}+\Delta_{\| \cdot} \cdot \Delta_{\perp}^{2}+\Delta_{\|}^{3}+\Delta_{\|} \cdot S_{\perp}^{2}$ \\
\hline$J_{\|}$or $M_{\text {rem }}$ & 4th order \\
\hline 1 & $\Delta_{\perp} \cdot \Delta_{\|} \cdot S_{\perp} \cdot S_{\|}+\Delta_{\perp}^{4}+\Delta_{\|}^{4}+S_{\perp}^{4}+S_{\|}^{4}+\Delta_{\perp}^{2} \cdot \Delta_{\|}^{2}+\Delta_{\perp}^{2} \cdot S_{\perp}^{2}+\Delta_{\perp}^{2} \cdot S_{\|}^{2}+\Delta_{\|}^{2} \cdot S_{\perp}^{2}+\Delta_{\|}^{2} \cdot S_{\|}^{2}+S_{\perp}^{2} \cdot S_{\|}^{2}$ \\
\hline$\delta m$ & $S_{\perp} \cdot \Delta_{\perp}^{3}+\Delta_{\|} \cdot S_{\|}^{3}+\Delta_{\|} \cdot S_{\|} \cdot \Delta_{\perp}^{2}+S_{\perp} \cdot \Delta_{\perp} \cdot S_{\|}^{2}+\Delta_{\|}^{3} \cdot S_{\|}+S_{\perp}^{3} \cdot \Delta_{\perp}+\Delta_{\|}^{2} \cdot S_{\perp} \cdot \Delta_{\perp}+\Delta_{\|} \cdot S_{\perp}^{2} \cdot S_{\|}$ \\
\hline
\end{tabular}

For the discussion below, we will denote terms in $J$ independent of $\delta m$ (or dependent on even powers of $\delta m$ ) by $J(1)$ and terms proportional to odd powers by $J(\delta m)$. The expansion terms reported in Tables $\mathrm{V}$ and $\mathrm{VI}$ are related to each other (which is a consequence of the symmetries in Table IV]. For example, all terms for the components of $\vec{J}$, with the exception of the even-order spin dependent terms of $J_{\|}(1)$, can be obtained from the terms in $J_{\perp}(1)$ under the following transformations

$$
\begin{array}{ccc}
J_{\perp}(1) \leftrightarrow J_{\perp}(\delta m) & \text { under } & {\left[\left(S_{\perp}, \Delta_{\perp}\right) \leftrightarrow\left(\Delta_{\perp}, S_{\perp}\right)\right] .(34} \\
J_{\perp}(\delta m) \leftrightarrow J_{\|}(\delta m) & \text { under } & {\left[\left(\Delta_{\perp}, \Delta_{\|}\right) \leftrightarrow\left(\Delta_{\|}, \Delta_{\perp}\right)\right] .(35}
\end{array}
$$

and for the odd-power in the spin variables only

$$
J_{\perp}(1) \leftrightarrow J_{\|}(1) \quad \text { under } \quad\left[\left(S_{\perp}, S_{\|}\right) \leftrightarrow\left(S_{\|}, S_{\perp}\right)\right] .
$$

The even-power (in the spin) terms in $J_{\|}(1)$ can be obtained from $J_{\perp}(1)$, but the process is more complex. Basically, one needs to take all terms in $J_{\|}(1)$ that are at least linear in $S_{\perp}$ and then divide by $S_{\perp}$. This means that there is a direct relationship between the even-order terms in $J_{\|}(1)$ and the next higher-order term in $J_{\perp}(1)$. This relationship also holds for the odd-order terms and $\delta m$ terms, but Eqs. 34- 36 are much more straightforward. It remains to be seen if the corresponding coefficients can be obtained (at least approximated) using these transformations. 


\section{Modeling the equal-mass case}

Using the above properties, and limiting our expansion to fourth-order in the spin variables, we find that there are 16 terms that contribute to the perpendicular component of the final angular momentum in the equal-mass case (i.e. $\left.J_{\perp}(1)\right)$. We can regroup all these terms in the following symbolic form

$$
\begin{aligned}
J_{\perp}= & S_{\perp} \cdot\left(1+\Delta_{\perp}^{2}+\cdots\right) \cdot\left(1+\Delta_{\|}^{2}+\cdots\right) . \\
& .\left(1+S_{\perp}^{2}+\cdots\right) \cdot\left(1+S_{\|}+S_{\|}^{2}+S_{\|}^{3}+\cdots\right)+ \\
& +\Delta_{\perp} \cdot \Delta_{\|} \cdot\left(1+\Delta_{\perp}^{2}+\cdots\right) \cdot\left(1+\Delta_{\|}^{2}+\cdots\right) . \\
& .\left(1+S_{\perp}^{2}+\cdots\right) .\left(1+S_{\|}+S_{\|}^{2}+\cdots\right) .
\end{aligned}
$$

Similar expansions can be generated for $J_{\perp}(\delta m)$ and $J_{\|}(\delta m)$ using properties (34) and (35).

Because the perpendicular components of $\vec{S}$ and $\vec{\Delta}$ are constructed by taking an inner product with unit vectors in the plane, these components vary sinusoidally under rotation of the directions of $\vec{S}$ and $\vec{\Delta}$. Thus $S_{\perp}=\left|S_{\perp}\right| \cos \varphi$, etc., where we use the notation $\left|S_{\perp}\right|$ to denote the magnitude of the projection of $\vec{S}$ onto the orbital plane. Indeed, each term linear in the subindex $\perp$ would lead to a $\cos \left(\varphi+\varphi_{[]}\right)$dependence $\left(\varphi_{[]}\right.$denotes a constant that, in principle, is different for each term). In our expansion above, there are ten terms directly proportional to $\cos \left(\varphi+\varphi_{[]}\right)$

$$
\begin{aligned}
J_{\perp}^{\cos \varphi}= & {\left[S_{\perp} \cdot\left(1+\Delta_{\|}^{2}+\cdots\right) \cdot\left(1+S_{\|}+S_{\|}^{2}+S_{\|}^{3}+\cdots\right)\right.} \\
& +\Delta_{\perp} \cdot \Delta_{\|} \cdot\left(1+\Delta_{\|}^{2}+\cdots\right) \\
& \left.\times\left(1+S_{\|}+S_{\|}^{2}+\cdots\right)\right] .
\end{aligned}
$$

In the same fashion we can regroup the 16 terms contributing up to the fourth order expansion of $J_{\|}$(or $\left.M_{\text {rem }}\right)$

$$
\begin{aligned}
J_{\|}= & \left(1+\Delta_{\perp}^{2}+\Delta_{\perp}^{4}+\cdots\right) \cdot\left(1+\Delta_{\|}^{2}+\Delta_{\|}^{4}+\cdots\right) . \\
& .\left(1+S_{\perp}^{2}+S_{\perp}^{4}+\cdots\right) .\left(1+S_{\|}+S_{\|}^{2}+S_{\|}^{3}+S_{\|}^{4}+\cdots\right) \\
& +\Delta_{\perp} \cdot \Delta_{\|} \cdot S_{\perp} \cdot\left(1+\Delta_{\perp}^{2}+\cdots\right) .\left(1+\Delta_{\|}^{2}+\cdots\right) \\
& .\left(1+S_{\perp}^{2}+\cdots\right) .\left(1+S_{\|}+\cdots\right) .
\end{aligned}
$$

Nine terms are independent of $\perp$ components of $\vec{S}$ or $\vec{\Delta}$ and are thus independent of $\cos (\varphi)$. The next set of terms are quadratic in the subindex $\perp$. We may expect that these quadratic terms lead a $\cos ^{2} \varphi$ dependence. However, they actually lead to both terms proportional to $\cos ^{2} \varphi$ and terms independent of $\cos \varphi$. To see why, consider the term $S_{\perp}^{2}$. This should really be written as $\left(\vec{S} \cdot \hat{n}_{1}\right)\left(\vec{S} \cdot \hat{n}_{2}\right)=\vec{S}_{\perp} \cos \phi \vec{S}_{\perp} \cos \left(\phi-\phi_{0}\right)$, where $\phi_{0}$ is the angle between $\hat{n}_{1}$ and $\hat{n}_{2}$. We then have $\left(\vec{S} \cdot \hat{n}_{1}\right)\left(\vec{S} \cdot \hat{n}_{2}\right)=S_{\perp}^{2} \cos ^{2} \varphi+S_{\perp}^{2}\left(1-\cos \phi_{0}\right) / 2$, where $\varphi=\phi-\phi_{0} / 2$ and $\phi_{0}$ is independent of orientation. These terms are

$$
\begin{array}{r}
J_{\|}^{1+\cos ^{2} \varphi}=\left(1+\Delta_{\|}^{2}+\Delta_{\|}^{4}+\cdots\right) \\
\times\left(1+S_{\|}+S_{\|}^{2}+S_{\|}^{3}+S_{\|}^{4}+\cdots\right) \\
+\left[\left(\Delta_{\perp}^{2}+S_{\perp}^{2}\right)\left(1+\Delta_{\|}^{2}+\cdots\right)\left(1+S_{\|}+S_{\|}^{2}+\cdots\right)\right. \\
\left.+\Delta_{\perp} \cdot \Delta_{\|} \cdot S_{\perp}\left(1+S_{\|}+\cdots\right)\right] .
\end{array}
$$

With an analogous expression for the final mass of the merged black hole $M_{\text {rem }}$.

Note that we actually fit to the magnitude of the remnant spin $m^{2} \alpha_{\text {rem }}^{2}=\left(J_{\perp}^{\cos \varphi}\right)^{2}+\left(J_{\|}^{1+\cos ^{2} \varphi}\right)^{2}$ which contains terms proportional to $(\cos \varphi)^{0},(\cos \varphi)^{2}, \cdots$.

\section{FITTING FULL NUMERICAL RESULTS}

We fit the total radiated mass and final remnant spin as a function of initial configuration for the SP and UD configurations described above, the hangup kick configurations (we will denote these configurations with HK) described in [14, 34, and the $\mathrm{N}$ configurations of [17. The HK configurations are equal-mass, equal-spin-magnitude configurations where the components of the spins along $\vec{L}$ are equal, and the components in the orbital plane are equal in magnitude but opposite in direction. We evolved two types of HK configurations: one with a dimensionless spin of 0.7 and the other with a dimensionless spin of 0.9 . We denote the latter by HK9 in the tables below. The $\mathrm{N}$ configurations have equal-mass $\mathrm{BHs}$ with one spinning and the other nonspinning. The SP configurations have $\vec{S} \neq 0$ but $\vec{\Delta}=0$, while the HK configurations have $\vec{S}_{\perp}=0$ and $\Delta_{\|}=0$. For both families, $S_{\|} \neq 0$, while for the UD configurations, $S_{\|}=0$. In addition, we use the results of the $\mathrm{L}, \mathrm{K}$, and $\mathrm{S}$ configurations of 17] to verify the accuracy of our model. The $\mathrm{K}$ configurations are equal-mass, equal-spin magnitude configurations where the components of the spin along $\vec{L}$ are equal in magnitude and opposite in direction and the components in the plane are equal in both magnitude and direction. The $\mathrm{L}$ configurations are again equal-mass, equal-spin magnitude configurations, but here, initially, one BH spin is aligned with $\vec{L}$ and the other lies in the orbital plane. Finally, the $\mathrm{S}$ configurations are equal-mass, equal spin magnitude configurations, where, initially, the components of the spin along $\vec{L}$ are equal in magnitude and opposite in direction and the components in the plane are also equal in magnitude and opposite in direction. For the N, L, K, and S configurations the dimensionless spin of the BHs (when not zero) was set to 0.8 .

Here we are interested in the total mass loss of the binary, starting from an infinite separation

$$
\delta \mathcal{M}=\frac{M_{1}^{\infty}+M_{2}^{\infty}-M_{\mathrm{rem}}}{M_{1}^{\infty}+M_{2}^{\infty}}
$$

(where $M_{1,2}^{\infty}$ are the masses of two BHs at infinite separation) and the final remnant spin $\alpha_{\text {rem }}$. We approximate 
$\delta \mathcal{M}$ by

$$
\delta \mathcal{M} \approx \frac{M_{1}+M_{2}-M_{\mathrm{rem}}}{M_{1}+M_{2}},
$$

where $M_{\text {rem }}$ is the remnant BH's mass and $M_{1}$ and $M_{2}$ are the initial masses of the two BHs in the binary (which is a good approximation for their masses when the binary was infinitely separated due to the near constancy of the BH mass during the inspiral, as seen in, e.g., Fig 50.

The measured values of $\delta \mathcal{M}$ and $\alpha_{\text {rem }}$ for the SP and UD configurations are given in Table XV] We found that the values of $\delta \mathcal{M}$ and $\alpha_{\text {rem }}$ obtained by the IH formalism were significantly more accurate than the radiation-based measures of these quantities (likely due to the resolution we used in the wave zone and to our placement of the extraction spheres). Hence, for all fits, we use the IH results.

Our fitting procedure is as follows. For each set of simulations with the same (initial) inclination angle $\theta$, we fit $\delta \mathcal{M}$ and $\alpha_{\text {rem }}^{2}$ to

$$
\begin{gathered}
\delta \mathcal{M}=E_{c}+E_{\phi} \cos \left(\varphi-\varphi_{e}\right)^{2}, \\
\delta \mathcal{M}=E_{c}^{\prime}+E_{\phi}^{\prime} \cos \left(2 \varphi-2 \varphi_{e}^{\prime}\right), \\
\alpha_{\mathrm{rem}}^{2}=A_{c}+A_{\phi} \cos \left(\varphi-\varphi_{a}\right)^{2}, \\
\alpha_{\mathrm{rem}}^{2}=A_{c}^{\prime}+A_{\phi}^{\prime} \cos \left(2 \varphi-2 \varphi_{a}^{\prime}\right),
\end{gathered}
$$

where $E_{c}, E_{\phi}, \varphi_{e}, A_{c}, A_{\phi}$, and $\varphi_{a}$ are fitting constants. While the expansion $C_{1}+C_{2} \cos ^{2} \phi$ is mathematically equivalent to the expansion $C_{1}^{\prime}+C_{2}^{\prime} \cos 2 \phi$ (i.e. $C_{1}^{\prime}=$ $C_{1}+C_{2} / 2$ and $C_{2}^{\prime}=C_{2} / 2$ ), we find that the constant term $C_{1}^{\prime}$ is better modeled as a function of $\theta$, as shown in Figs. 15, 16, 17, and 18. Fits to $A_{\phi}$ show substantial scatter when compared to other quantities. The most likely explanation for the scatter is that there were not enough $\phi$ configurations to accurately extract $A_{\phi}$.

As an aside, we note that we actually measure the angle $\phi$ using the techniques of [64. Briefly, we measure the spin direction of each $\mathrm{BH}$ near merger in a frame rotated in such a way that the late-time trajectories overlap. The angle $\phi$ is then the orientation of the spin of $B H 1$ in this frame.

We then fit $E_{c}, E_{c}^{\prime}, A_{c}$, and $A_{c}^{\prime}$ as functions of $\theta$, as follows. First we note that at $\theta=0$ and $\theta=$ $180^{\circ}$, we should obtain the associated hangup energies and final remnant spins. In addition, because the resulting formulas should be an even function of $\theta$ (i.e., $\pm \theta$ are equivalent), we fit these functions to the form $f(\theta)=\sin ^{2} \theta\left(c_{0}+c_{1} \cos \theta+c_{2} \cos ^{2} \theta+\cdots\right)$. However, since $S_{\perp} \propto \sin \theta$ and $S_{\|} \propto \cos \theta$, our actual fitting functions are

$$
\begin{aligned}
& E_{c}=E_{\mathrm{hu}}+\left(\frac{S_{\perp}}{m^{2}}\right)^{2}\left(e_{1}+e_{2}\left(\frac{S_{\|}}{m^{2}}\right)+e_{3}\left(\frac{S_{\|}}{m^{2}}\right)^{2}\right), \\
& A_{c}=A_{\mathrm{hu}}^{2}+\left(\frac{S_{\perp}}{m^{2}}\right)^{2}\left(a_{1}+a_{2}\left(\frac{S_{\|}}{m^{2}}\right)+a_{3}\left(\frac{S_{\|}}{m^{2}}\right)^{2}\right)(, 45)
\end{aligned}
$$

where $E_{\mathrm{hu}}$ is the radiated energy for a pure hangup configuration with the same $S_{\|}$and $A_{\text {hu }}$ is the remnant spin
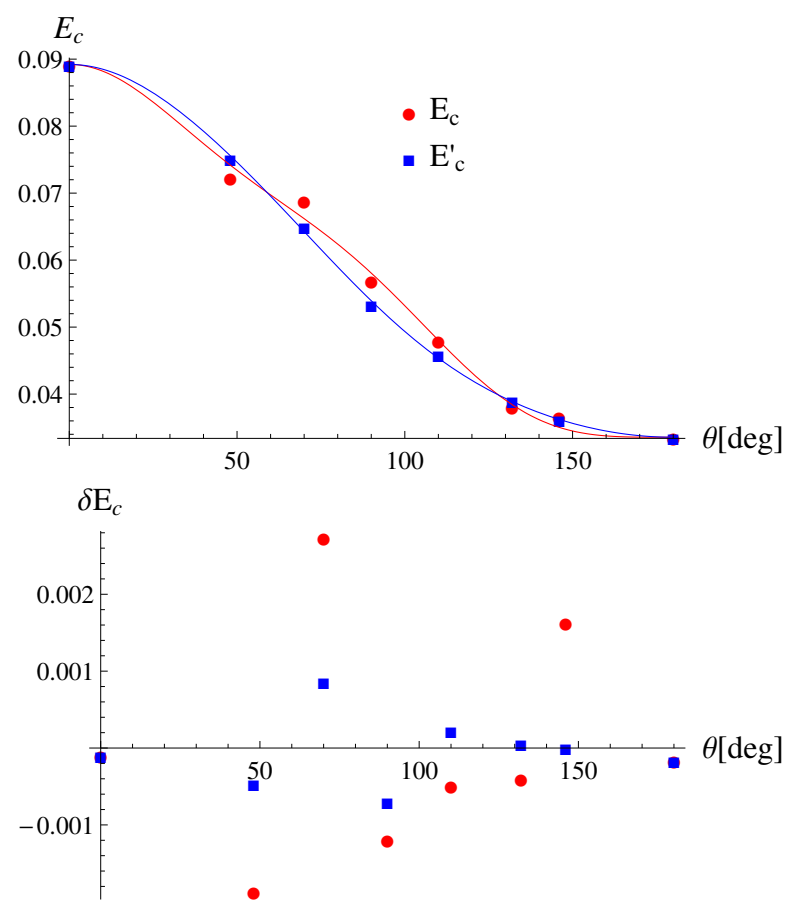

FIG. 15: Fits of $E_{c}$ and $E_{c}^{\prime}$ versus $\theta$ for the SP configurations. The upper panel shows the data and fit, while the lower shows the residuals. Note that the scatter in $E_{c}^{\prime}$ is substantially better than for $E_{c}$.

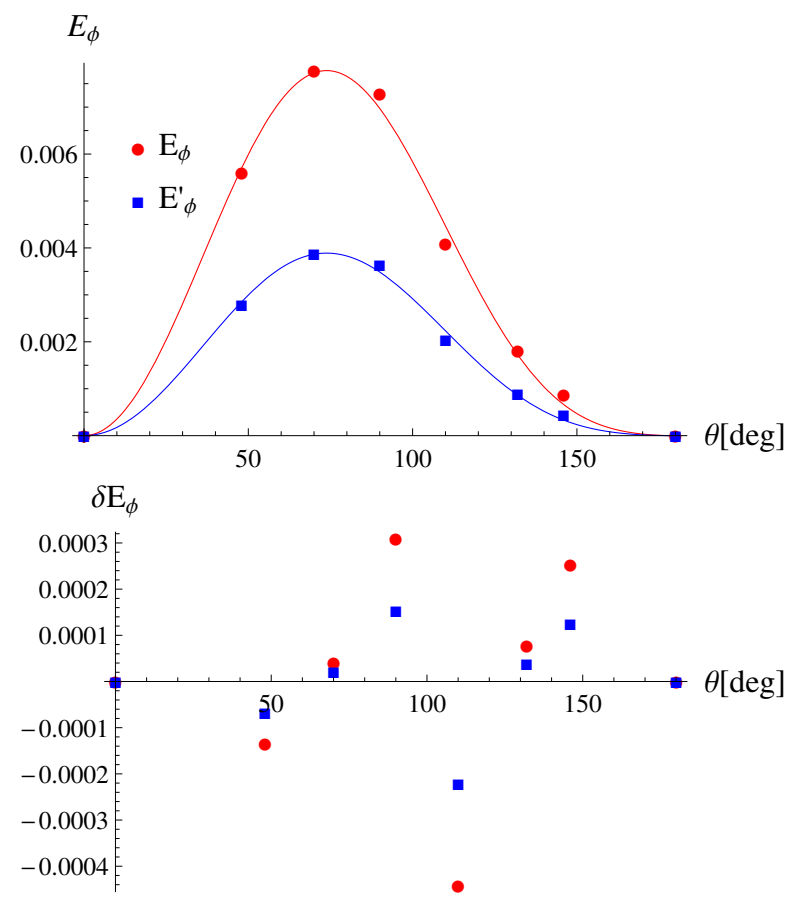

FIG. 16: Fits of $E_{\phi}$ and $E_{\phi}^{\prime}$ (note that $E_{\phi}^{\prime}=E_{\phi} / 2$ identically) versus $\theta$ for the SP configurations. The upper panel shows the data and fits, while the lower shows the residuals. 

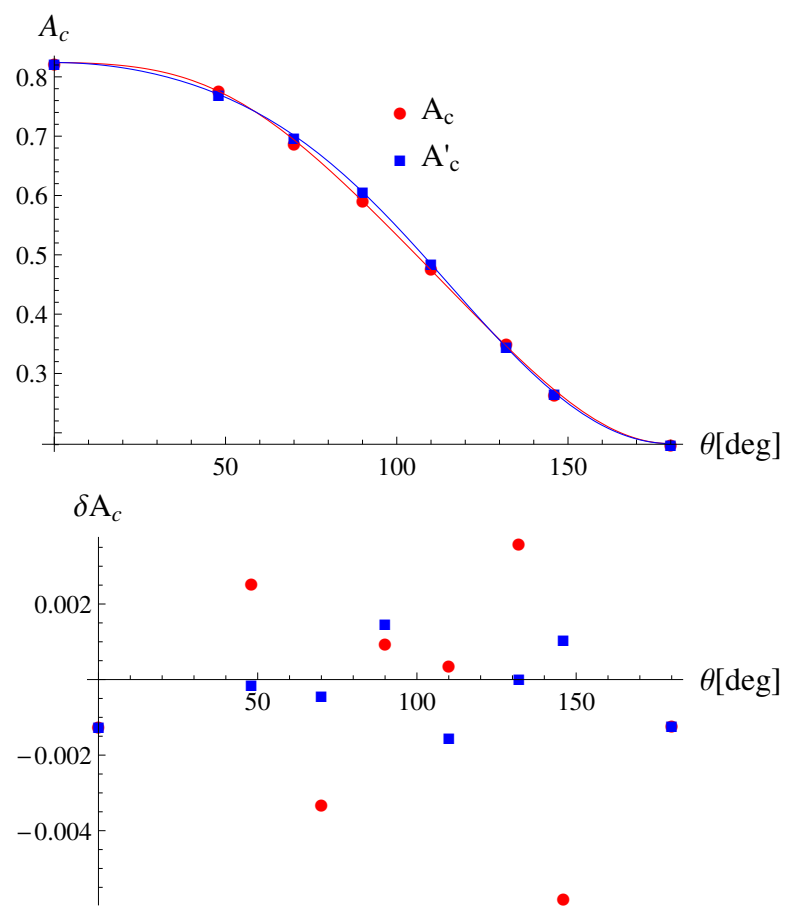

FIG. 17: Fits of $A_{c}$ and, $A_{c}^{\prime}$ versus $\theta$ for the SP configurations. The upper panel shows the data and fit, while the lower shows the residuals.
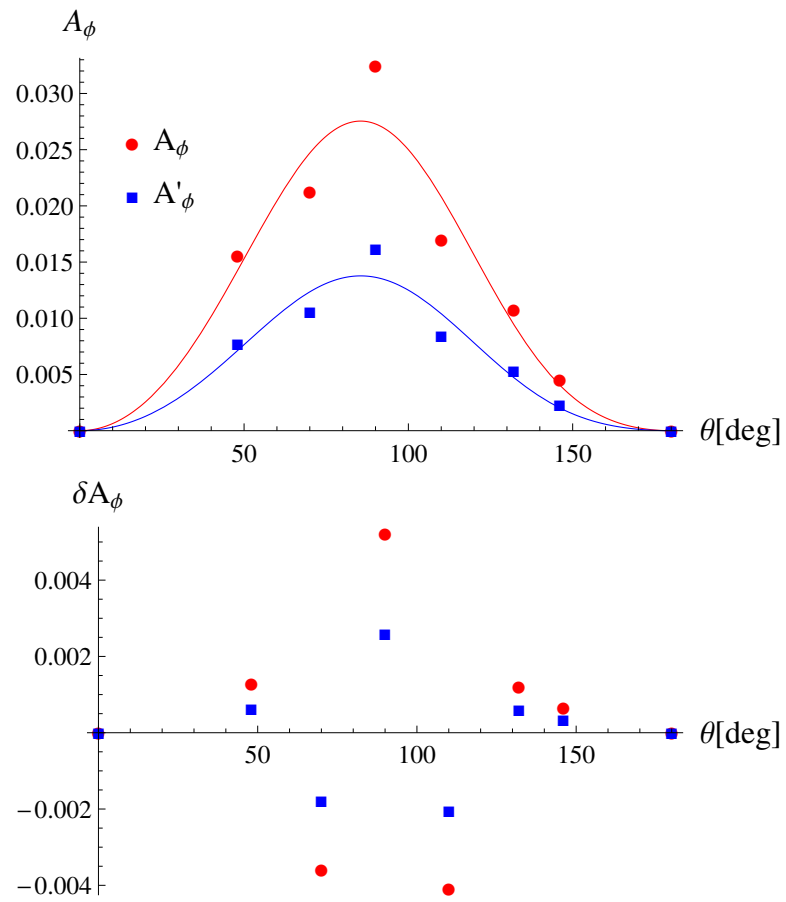

FIG. 18: Fits of $A_{\phi}$ and $A_{\phi}^{\prime}$ versus $\theta$ for the SP configurations (note that $A_{\phi}^{\prime}=A_{\phi} / 2$ identically). The upper panel shows the data and fits, while the lower shows the residuals. Note that the fits to $A_{\phi}$ and $A_{\phi}^{\prime}$ are rather poor. for the same hangup configuration. We use the formula provided in 60] for both $E_{\mathrm{hu}}(\theta)$ and $A_{\mathrm{hu}}(\theta)$. The fitting constants (with errors) are given in Table VIII. In addition, we fit $E_{\phi}$ and $A_{\phi}$ to the forms

$$
\begin{gathered}
E_{\phi}=\left(\frac{S_{\perp}}{m^{2}}\right)^{2}\left(\mathcal{E}_{1}+\mathcal{E}_{2}\left(\frac{S_{\|}}{m^{2}}\right)+\mathcal{E}_{3}\left(\frac{S_{\|}}{m^{2}}\right)^{2}\right), \\
A_{\phi}=\left(\frac{S_{\perp}}{m^{2}}\right)^{2}\left(\mathcal{A}_{1}+\mathcal{A}_{2}\left(\frac{S_{\|}}{m^{2}}\right)+\mathcal{A}_{3}\left(\frac{S_{\|}}{m^{2}}\right)^{2}\right),
\end{gathered}
$$

These fitting constants (with errors) for the SP configurations are given in Table VIII as well. Note that we do not include terms proportional to $\Delta_{\perp}^{4}$. In principle these terms should appear, but based the results from the hangup kick effect [34, where we found the subleading terms in $\Delta_{\perp}$ do not contribute significantly to the recoil, we omit these terms here, as well. We test the validity of this assumption later below by comparing the predictions of our formula with the results from over 100 additional configurations. 
TABLE VII: Fitting parameters for the radiated energy and final remnant spin for each TH family of SP configurations. The $\varphi_{e}$ and $\varphi_{a}$ parameters are not shown (prime and unprimed).

\begin{tabular}{l|llll}
\hline \hline Family & $E_{c}$ & $E_{\phi}$ & $E_{c}^{\prime}$ & $E_{\phi}^{\prime}$ \\
SPTH48 & $0.07223 \pm 0.00066$ & $0.00560 \pm 0.00089$ & $0.07503 \pm 0.00040$ & $0.00280 \pm 0.00045$ \\
SPTH70 & $0.06874 \pm 0.00091$ & $0.00779 \pm 0.00107$ & $0.06484 \pm 0.00040$ & $0.00389 \pm 0.00054$ \\
SPTH90 & $0.05687 \pm 0.00035$ & $0.00729 \pm 0.00060$ & $0.05323 \pm 0.00014$ & $0.00364 \pm 0.00030$ \\
SPTH110 & $0.04782 \pm 0.00037$ & $0.00410 \pm 0.00055$ & $0.04577 \pm 0.00018$ & $0.00205 \pm 0.00027$ \\
SPTH132 & $0.03808 \pm 0.00006$ & $0.00182 \pm 0.00008$ & $0.03898 \pm 0.00003$ & $0.00091 \pm 0.00004$ \\
SPTH146 & $0.03660 \pm 0.00005$ & $0.00089 \pm 0.00008$ & $0.03615 \pm 0.00003$ & $0.00045 \pm 0.00004$ \\
\hline Family & $A_{c}$ & $A_{\phi}$ & $A_{c}^{\prime}$ & $A_{\phi}^{\prime}$ \\
SPTH48 & $0.77775 \pm 0.00451$ & $0.01563 \pm 0.00602$ & $0.76994 \pm 0.00262$ & $0.00781 \pm 0.00301$ \\
SPTH70 & $0.68821 \pm 0.00290$ & $0.02132 \pm 0.00291$ & $0.69887 \pm 0.00220$ & $0.01066 \pm 0.00146$ \\
SPTH90 & $0.59141 \pm 0.00642$ & $0.03252 \pm 0.01126$ & $0.60767 \pm 0.00261$ & $0.01626 \pm 0.00563$ \\
SPTH110 & $0.47790 \pm 0.00233$ & $0.01700 \pm 0.00338$ & $0.48640 \pm 0.00111$ & $0.00850 \pm 0.00169$ \\
SPTH132 & $0.35056 \pm 0.00164$ & $0.01079 \pm 0.00209$ & $0.34516 \pm 0.00088$ & $0.00539 \pm 0.00105$ \\
SPTH146 & $0.26443 \pm 0.00055$ & $0.00462 \pm 0.00081$ & $0.26674 \pm 0.00030$ & $0.00231 \pm 0.00041$ \\
\hline \hline
\end{tabular}

TABLE VIII: Fitting parameters for coefficient $E_{c}$ and $A_{c}$ as a function of $S_{\perp}$ and $S_{\|}$for the SP configurations.

\begin{tabular}{ll|ll}
\hline \hline$e_{1}$ & $0.0613 \pm 0.0080$ & $e_{1}^{\prime}$ & $0.0356 \pm 0.0025$ \\
$e_{2}$ & $0.0764 \pm 0.0388$ & $e_{2}^{\prime}$ & $0.0960 \pm 0.0122$ \\
$e_{3}$ & $-0.3842 \pm 0.2133$ & $e_{3}^{\prime}$ & $0.1217 \pm 0.0671$ \\
$\mathcal{E}_{1}$ & $0.0434 \pm 0.0013$ & $\mathcal{E}_{1}^{\prime}$ & $0.0217 \pm 0.0006$ \\
$\mathcal{E}_{2}$ & $0.0839 \pm 0.0061$ & $\mathcal{E}_{2}^{\prime}$ & $0.0419 \pm 0.0030$ \\
$\mathcal{E}_{3}$ & $-0.0214 \pm 0.0334$ & $\mathcal{E}_{3}^{\prime}$ & $-0.0107 \pm 0.0167$ \\
$a_{1}$ & $0.7422 \pm 0.0168$ & $a_{1}^{\prime}$ & $0.8401 \pm 0.0061$ \\
$a_{2}$ & $-0.2588 \pm 0.0812$ & $a_{2}^{\prime}$ & $-0.3277 \pm 0.0294$ \\
$a_{3}$ & $1.3068 \pm 0.4461$ & $a_{3}^{\prime}$ & $-0.6088 \pm 0.1616$ \\
$\mathcal{A}_{1}$ & $0.1699 \pm 0.0158$ & $\mathcal{A}_{1}^{\prime}$ & $0.0849 \pm 0.0079$ \\
$\mathcal{A}_{2}$ & $0.1000 \pm 0.0764$ & $\mathcal{A}_{2}^{\prime}$ & $0.0500 \pm 0.0382$ \\
$\mathcal{A}_{3}$ & $-0.5023 \pm 0.4197$ & $\mathcal{A}_{3}^{\prime}$ & $-0.2512 \pm 0.2099$ \\
\hline \hline
\end{tabular}

The new formula for $A_{c}$ agrees quite will with the ansatz in [21]. In [21], we conjectured, based on most of the above runs, that the final remnant spin could be obtained from

$$
\alpha_{\mathrm{rem}}^{2}=\alpha_{\mathrm{hu}}^{2}+\frac{S_{\perp}^{2}}{m^{2}}
$$

The idea was that the in-plane spin was preserved and therefore contributed entirely to the final remnant spin. The other components of the spin arising from the hangup effect. Here we find,

$\alpha_{\mathrm{rem}}^{2}=\alpha_{\mathrm{hu}}^{2}+0.84 \frac{S_{\perp}^{2}}{m^{2}}+0.085 \frac{S_{\perp}^{2}}{m^{2}} \cos (2 \phi)+($ small corrections $)$

We thus see that, depending on orientation between $3.8 \%$ and $13.1 \%$ (with an average of $8.4 \%$ ) of the in-plane spin is actually lost to radiation.

We use an essentially identical procedure to fit the mass loss and remnant spin from the hangup kick (HK) configurations. The main difference here is that $\sin \theta$ is proportional to $\Delta_{\perp}$, rather than to $S_{\perp}(\cos \theta$ is still proportional to $S_{\|}$).
TABLE IX: Fitting parameters for coefficient $E_{c}, E_{\phi}, A_{c}$, and $A_{\phi}$ as functions of $\Delta_{\perp}$ and $S_{\|}$for the HK configurations. Note how the $\zeta_{i}$ coefficients are consistent with zero.

\begin{tabular}{ll|ll}
\hline \hline$\epsilon_{1}$ & $0.0081 \pm 0.0062$ & $\epsilon_{1}^{\prime}$ & $0.0043 \pm 0.0012$ \\
$\epsilon_{2}$ & $0.0075 \pm 0.0110$ & $\epsilon_{2}^{\prime}$ & $0.0050 \pm 0.0021$ \\
$\epsilon_{3}$ & $-0.0984 \pm 0.1394$ & $\epsilon_{3}^{\prime}$ & $-0.0090 \pm 0.0263$ \\
$\tilde{E}_{1}$ & $0.0051 \pm 0.0004$ & $\tilde{E}_{1}^{\prime}$ & $0.0025 \pm 0.0002$ \\
$\tilde{E}_{2}$ & $0.0119 \pm 0.0007$ & $\tilde{E}_{2}^{\prime}$ & $0.0060 \pm 0.0004$ \\
$\tilde{E}_{3}$ & $0.0316 \pm 0.0092$ & $\tilde{E}_{3}^{\prime}$ & $0.0158 \pm 0.0046$ \\
$\zeta_{1}$ & $-0.0028 \pm 0.0121$ & $\zeta_{1}^{\prime}$ & $-0.0209 \pm 0.0070$ \\
$\zeta_{2}$ & $-0.0007 \pm 0.0213$ & $\zeta_{2}^{\prime}$ & $-0.0381 \pm 0.0124$ \\
$\zeta_{3}$ & $-0.1420 \pm 0.2690$ & $\zeta_{3}^{\prime}$ & $0.0429 \pm 0.1568$ \\
$\tilde{A}_{1}$ & $0.0234 \pm 0.0017$ & $\tilde{A}_{1}^{\prime}$ & $0.0117 \pm 0.0008$ \\
$\tilde{A}_{2}$ & $0.0421 \pm 0.0030$ & $\tilde{A}_{2}^{\prime}$ & $0.0210 \pm 0.0015$ \\
$\tilde{A}_{3}$ & $0.0561 \pm 0.0373$ & $\tilde{A}_{3}^{\prime}$ & $0.0281 \pm 0.0187$ \\
\hline \hline
\end{tabular}

The fitting function for the HK runs has the form

$$
\begin{aligned}
& E_{c}=E_{\mathrm{hu}}+\left(\frac{\Delta_{\perp}}{m^{2}}\right)^{2}\left(\epsilon_{1}+\epsilon_{2}\left(\frac{S_{\|}}{m^{2}}\right)+\epsilon_{3}\left(\frac{S_{\|}}{m^{2}}\right)^{2}\right), \\
& E_{\phi}=\left(\frac{\Delta_{\perp}}{m^{2}}\right)^{2}\left(\tilde{E}_{1}+\tilde{E}_{2}\left(\frac{S_{\|}}{m^{2}}\right)+\tilde{E}_{3}\left(\frac{S_{\|}}{m^{2}}\right)^{2}\right), \\
& A_{c}=A_{\mathrm{hu}}^{2}+\left(\frac{\Delta_{\perp}}{m^{2}}\right)^{2}\left(\zeta_{1}+\zeta_{2}\left(\frac{S_{\|}}{m^{2}}\right)+\zeta_{3}\left(\frac{S_{\|}}{m^{2}}\right)^{2}\right), \\
& A_{\phi}=\left(\frac{\Delta_{\perp}}{m^{2}}\right)^{2}\left(\tilde{A}_{1}+\tilde{A}_{2}\left(\frac{S_{\|}}{m^{2}}\right)+\tilde{A}_{3}\left(\frac{S_{\|}}{m^{2}}\right)^{2}\right) .
\end{aligned}
$$

The fitted constants are provided in Table IX. Note here how the correction to $A_{c}$ (above the hangup correction) is consistent with zero.

Finally, using the N configurations (only one of the black holes is spinning) of Ref. [17, we can attempt to fit terms in the radiated mass and final spin proportional to $\Delta_{\|}$. In order to do this, we must make an assumption regarding how the $S_{\perp}^{2}$ terms and $\Delta_{\perp}^{2}$ terms interact. 
TABLE X: Fitting parameters for coefficient $E_{c}, E_{\phi}, A_{c}$, and $A_{\phi}$ as functions of $\Delta_{\|}$and $S_{\|}$for the N configurations

\begin{tabular}{ll|ll}
\hline \hline$k_{2}$ & 0 & $k_{2}^{\prime}$ & 0 \\
$k_{3}$ & 0 & $k_{3}^{\prime}$ & 0 \\
$k_{4}$ & $0.045 \pm 0.026$ & $k_{4}^{\prime}$ & $0.0174 \pm 0.0086$ \\
$m_{4}$ & 0 & $m_{4}^{\prime}$ & 0 \\
$n_{2}$ & 0 & $n_{2}^{\prime}$ & $-0.0308 \pm 0.0086$ \\
$n_{3}$ & 0 & $n_{3}^{\prime}$ & 0 \\
$n_{4}$ & 0 & $n_{4}^{\prime}$ & 0 \\
$p_{4}$ & 0 & $p_{4}^{\prime}$ & 0 \\
\hline \hline
\end{tabular}

That is, each one has an oscillatory behavior of the form $\cos ^{2}(\phi-c)$, where $c$ is some constant phase. Here, we assume that the two phases are equal. This will introduce an error into our fits. However, as we show below, this error is of the same order of magnitude as the error in the $S_{\perp}^{2}$ and $\Delta_{\perp}^{2}$ terms themselves.

Thus, to fit the $\Delta_{\|}$terms with the $\mathrm{N}$ configuration, we take the coefficients in Tables VIII, IX, and II] as exact and then fit the energy radiated and final remnant spins to the forms

$$
\begin{aligned}
E_{c}= & E_{c}^{\Delta_{\|}=0}+\left(\frac{\Delta_{\|}}{m^{2}}\right)^{2}\left(E_{2}+k_{2} S_{\|}+k_{3} S_{\|}^{2}\right) \\
& +k_{4} \Delta_{\|} \Delta_{\perp} S_{\perp}+E_{4} \Delta_{\|}^{4} \\
E_{\phi}= & E_{\phi}^{\Delta_{\|}=0}+m_{4} \Delta_{\|} \Delta_{\perp} S_{\perp} \\
A_{c}= & A_{c}^{\Delta_{\|}=0}+\left(\frac{\Delta_{\|}}{m^{2}}\right)^{2}\left(A_{2}+n_{2} S_{\|}+n_{3} S_{\|}^{2}\right) \\
& +n_{4} \Delta_{\|} \Delta_{\perp} S_{\perp} \\
A_{\phi}= & A_{\phi}^{\Delta_{\|}=0}+p_{4} \Delta_{\|} \Delta_{\perp} S_{\perp} .
\end{aligned}
$$

Note here that $E_{2}, E_{4}$, and $A_{2}$ were taken to be the values given in Table II. Here, we found that most coefficients were consistent with zero (i.e. the confidence interval contained zero). We removed those coefficients from the fitting functions and then fit again. Our results are summarized in Table $\mathrm{X}$

Generalizing these results from the $\mathrm{N}$ configurations introduces a problem because there is a sign ambiguity in $\Delta_{\|} \Delta_{\perp} S_{\perp}$. To understand the source of this ambiguity, we need to consider how this term arises. In general, these terms come from interaction terms of the form

$$
(\vec{\Delta} \cdot \hat{L})\left(\vec{\Delta} \cdot \hat{e}_{\Delta}\right)\left(\vec{S} \cdot \hat{e}_{S}\right)
$$

If we align our coordinate system such that $\hat{e}_{\Delta}$ is aligned with the $x$ axis and $\hat{L}$ is aligned with the $z$ axis, then

$$
(\vec{\Delta} \cdot \hat{L})\left(\vec{\Delta} \cdot \hat{e}_{\Delta}\right)\left(\vec{S} \cdot \hat{e}_{S}\right)=\Delta_{\|}\left|\Delta_{\perp} \| S_{\perp}\right| \cos \phi \cos \left(\phi-\phi_{S}\right),
$$

where $\phi_{S}$ is the angle between $\hat{e}_{\Delta}$ and $\hat{e}_{S}$. This then becomes

$$
(1 / 2)\left(\cos \left(2 \phi-\phi_{S}\right)+\cos \phi_{S}\right)\left(\Delta_{\|}\left|\Delta_{\perp}\right|\left|S_{\perp}\right|\right)
$$

That is, there are terms proportional to $\cos (2 \phi-c)$ and a constant term proportional the $\cos \phi_{S}$. Under exchange of labels $(1 \leftrightarrow 2) \vec{\Delta}$ rotates by $180^{\circ}$ and, consequently, $\cos \phi_{S}$ changes sign. We absorb $\cos \phi_{S}$ into the fitting constant, but have no way of encoding the information about its sign. Later, we explore the effects of simply dropping these ambiguous terms from our model.

Our final formula in the equal-mass case is given by

$$
\begin{gathered}
E_{c}=E_{\mathrm{hu}}+\left(\frac{S_{\perp}}{m^{2}}\right)^{2}\left(e_{1}+e_{2}\left(\frac{S_{\|}}{m^{2}}\right)+e_{3}\left(\frac{S_{\|}}{m^{2}}\right)^{2}\right)+ \\
\left(\frac{\Delta_{\perp}}{m^{2}}\right)^{2}\left(\epsilon_{1}+\epsilon_{2}\left(\frac{S_{\|}}{m^{2}}\right)+\epsilon_{3}\left(\frac{S_{\|}}{m^{2}}\right)^{2}\right)+ \\
\left(\frac{\Delta_{\|}}{m^{2}}\right)^{2}\left(E_{2}+k_{2} S_{\|}+k_{3} S_{\|}^{2}\right)+ \\
k_{4} \Delta_{\|} \Delta_{\perp} S_{\perp}+E_{4} \Delta_{\|}^{4} \\
E_{\phi}=\left(\frac{S_{\perp}}{m^{2}}\right)^{2}\left(\mathcal{E}_{1}+\mathcal{E}_{2}\left(\frac{S_{\|}}{m^{2}}\right)+\mathcal{E}_{3}\left(\frac{S_{\|}}{m^{2}}\right)^{2}\right)+ \\
\left(\frac{\Delta_{\perp}}{m^{2}}\right)^{2}\left(\tilde{E}_{1}+\tilde{E}_{2}\left(\frac{S_{\|}}{m^{2}}\right)+\tilde{E}_{3}\left(\frac{S_{\|}}{m^{2}}\right)^{2}\right)+ \\
m_{4} \Delta_{\|} \Delta_{\perp} S_{\perp}
\end{gathered}
$$

$$
\begin{array}{r}
A_{c}=A_{\mathrm{hu}}^{2}+\left(\frac{S_{\perp}}{m^{2}}\right)^{2}\left(a_{1}+a_{2}\left(\frac{S_{\|}}{m^{2}}\right)+a_{3}\left(\frac{S_{\|}}{m^{2}}\right)^{2}\right)+ \\
\left(\frac{\Delta_{\perp}}{m^{2}}\right)^{2}\left(\zeta_{1}+\zeta_{2}\left(\frac{S_{\|}}{m^{2}}\right)+\zeta_{3}\left(\frac{S_{\|}}{m^{2}}\right)^{2}\right)+ \\
\left(\frac{\Delta_{\|}}{m^{2}}\right)^{2}\left(A_{2}+n_{2} S_{\|}+n_{3} S_{\|}^{2}\right) \\
A_{\phi}=\left(\frac{S_{\perp}}{m^{2}}\right)^{2}\left(\mathcal{A}_{1}+\mathcal{A}_{2}\left(\frac{S_{\|}}{m^{2}}\right)+\mathcal{A}_{3}\left(\frac{S_{\|}}{m^{2}}\right)^{2}\right)+ \\
\left(\frac{\Delta_{\perp}}{m^{2}}\right)^{2}\left(\tilde{A}_{1}+\tilde{A}_{2}\left(\frac{S_{\|}}{m^{2}}\right)+\tilde{A}_{3}\left(\frac{S_{\|}}{m^{2}}\right)^{2}\right)+ \\
p_{4} \Delta_{\|} \Delta_{\perp} S_{\perp},
\end{array}
$$

where $\delta \mathcal{M}=E_{c}+E_{\phi} \cos (2 \phi)$ and $\alpha_{\text {rem }}^{2}=A_{c}+A_{\phi} \cos (2 \phi)$ and the coefficients are given by the primed quantities in Tables II, VIII IX and X or $\delta \mathcal{M}=E_{c}+E_{\phi} \cos ^{2}(\phi)$ and $\alpha_{\mathrm{rem}}^{2}=A_{c}+A_{\phi} \cos ^{2}(\phi)$ and the coefficients are given by 
TABLE XI: The absolute and relative differences (AE and RE) for $E_{c}^{\prime}, E_{\phi}^{\prime}, A_{c}^{\prime}, A_{\phi}^{\prime}$, as measured directly by fitting to the simulation data and the predictions of Eqs. (48)-(51).

\begin{tabular}{|c|c|c|c|c|c|c|c|c|}
\hline Family & $\mathrm{AE}\left(E_{c}^{\prime}\right)$ & $\operatorname{RE}\left(E_{c}^{\prime}\right) \%$ & $\operatorname{AE}\left(E_{\phi}^{\prime}\right)$ & $\operatorname{RE}\left(E_{\phi}^{\prime}\right) \%$ & $\operatorname{AE}\left(A_{c}^{\prime}\right)$ & $\operatorname{RE}\left(A_{c}^{\prime}\right) \%$ & $\operatorname{AE}\left(A_{\phi}^{\prime}\right)$ & $\operatorname{RE}\left(A_{\phi}^{\prime}\right) \%$ \\
\hline SPTH0 & 0.00011 & \begin{tabular}{l|l}
0.122 \\
\end{tabular} & 0 & 0 & 0.00124 & \begin{tabular}{l|l}
0.151 \\
\end{tabular} & 0 & 0 \\
\hline SPTH48 & 0.00047 & 0.625 & 0.0000674 & 2.407 & 0.01121 & 1.455 & -0.00064 & -8.167 \\
\hline SPTH70 & -0.00085 & -1.314 & -0.0000209 & -0.536 & 0.00762 & 1.090 & 0.00179 & 16.783 \\
\hline SPTH90 & 0.00071 & 1.331 & -0.0001547 & -4.245 & -0.00149 & -0.245 & -0.00261 & -16.030 \\
\hline SPTH110 & -0.00021 & -0.458 & 0.0002206 & 10.755 & -0.00233 & -0.478 & 0.00204 & 23.968 \\
\hline SPTH132 & -0.00005 & -0.124 & -0.0000390 & -4.294 & -0.00277 & -0.802 & -0.00061 & -11.253 \\
\hline SPTH146 & $10^{-5}$ & 0.013 & -0.0001264 & -28.355 & -0.00224 & -0.840 & -0.00033 & -14.447 \\
\hline SPTH180 & 0.00018 & 0.531 & 0 & 0 & 0.00121 & 0.669 & 0 & 0 \\
\hline HK22.5 & 0.00034 & 0.435 & -0.0000474 & -9.474 & -0.00200 & -0.263 & -0.00023 & -12.523 \\
\hline HK45 & -0.00017 & -0.240 & $10^{-6}$ & 0.071 & 0.00097 & 0.142 & 0.00014 & 3.158 \\
\hline HK60 & 0.00003 & 0.048 & 0.0000170 & 1.116 & -0.00016 & -0.026 & -0.00005 & -0.814 \\
\hline HK120 & 0.00006 & 0.152 & -0.0000301 & -3.891 & -0.00039 & -0.119 & -0.00001 & -0.368 \\
\hline HK135 & -0.00007 & -0.187 & 0.0000327 & 6.846 & 0.00044 & 0.161 & 0.00002 & 0.816 \\
\hline HK9TH15 & -0.00045 & -0.458 & -0.0000770 & -14.759 & 0.01036 & 1.230 & -0.00090 & -38.694 \\
\hline НК9ТН30 & -0.00042 & -0.475 & -0.0000148 & -0.989 & 0.01273 & 1.597 & 0.00263 & 114.180 \\
\hline HK9TH60L & -0.00023 & -0.332 & $-5.6 * 10^{-6}$ & -0.196 & -0.01295 & -1.944 & -0.01431 & -56.678 \\
\hline UD00 & -0.00011 & -0.231 & 0 & 0 & 0.00016 & 0.033 & 0 & 0 \\
\hline UD60 & -0.00011 & -0.233 & 0 & 0 & 0.00017 & 0.036 & 0 & 0 \\
\hline UD70 & -0.00011 & -0.226 & 0 & 0 & 0.00017 & 0.036 & 0 & 0 \\
\hline UD80 & -0.00011 & -0.226 & 0 & 0 & 0.00021 & 0.045 & 0 & 0 \\
\hline UD85 & -0.00013 & -0.257 & 0 & 0 & 0.0001 & 0.021 & 0 & 0 \\
\hline NTH15 & -0.0001 & -0.154 & 0.0000228 & 22.374 & 0.00009 & 0.013 & 0.00003 & 6.638 \\
\hline NTH30 & -0.00003 & -0.044 & 0.0001060 & 30.942 & 0.00015 & 0.024 & 0.00009 & 6.264 \\
\hline NTH45 & 0.00006 & 0.104 & 0.0002808 & 49.797 & 0.00031 & 0.051 & -0.00004 & -1.196 \\
\hline NTH60 & 0.00017 & 0.302 & 0.0003417 & 41.263 & 0.00039 & 0.067 & 0.00092 & 27.088 \\
\hline NTH120 & 0.00024 & 0.529 & 0.0002547 & 49.014 & -0.00001 & -0.002 & 0.00065 & 22.629 \\
\hline NTH135 & -0.00019 & -0.453 & 0.0000261 & 8.508 & 0.00064 & 0.170 & -0.00010 & -6.273 \\
\hline NTH165 & 0.00010 & 0.258 & 0.0000393 & 105.030 & 0.00012 & 0.038 & 0.00017 & 93.699 \\
\hline S & 0.00010 & 0.205 & -0.0000629 & -7.169 & -0.00108 & -0.233 & 0.00023 & 6.555 \\
\hline $\mathrm{L}$ & 0.00008 & 0.118 & 0.0005827 & 44.456 & 0.00600 & 0.898 & 0.00151 & 32.595 \\
\hline КTH45 & -0.00076 & -1.450 & 0.0005348 & 44.254 & 0.00567 & 1.066 & 0.00037 & 5.665 \\
\hline KTH135 & -0.00069 & -1.371 & -0.0001528 & -23.034 & 0.00364 & 0.752 & -0.00035 & -14.783 \\
\hline
\end{tabular}

the unprimed quantities. As the angle $\phi$ is not known in general, we recommend using these formulas in statistical studies where one may assume a uniform (or perhaps biased) distribution in $\phi$. can be re-written as

$$
\begin{aligned}
\delta \mathcal{M}= & \delta \mathcal{M}_{\mathrm{hu}}+ \\
& \left(\frac{S_{\perp}}{m^{2}}\right)^{2}\left(e_{1}+e_{2}\left(\frac{S_{\|}}{m^{2}}\right)+e_{3}\left(\frac{S_{\|}}{m^{2}}\right)^{2}\right)+ \\
& \left(\frac{\Delta_{\perp}}{m^{2}}\right)^{2}\left(\epsilon_{1}+\epsilon_{2}\left(\frac{S_{\|}}{m^{2}}\right)+\epsilon_{3}\left(\frac{S_{\|}}{m^{2}}\right)^{2}\right)+ \\
& \left(\frac{\Delta_{\|}}{m^{2}}\right)^{2}\left(E_{2}+k_{2} \frac{S_{\|}}{m^{2}}+k_{3}\left(\frac{S_{\|}}{m^{2}}\right)^{2}\right)+ \\
& k_{4} \Delta_{\|} \Delta_{\perp} S_{\perp}+E_{4} \Delta_{\|}^{4}+ \\
& \left(\frac{S_{\perp} \cdot \hat{n}}{m^{2}}\right)^{2}\left(\mathcal{E}_{1}+\mathcal{E}_{2}\left(\frac{S_{\|}}{m^{2}}\right)+\mathcal{E}_{3}\left(\frac{S_{\|}}{m^{2}}\right)^{2}\right)+ \\
& \left(\frac{\Delta_{\perp} \cdot \hat{l}}{m^{2}}\right)^{2}\left(\tilde{E}_{1}+\tilde{E}_{2}\left(\frac{S_{\|}}{m^{2}}\right)+\tilde{E}_{3}\left(\frac{S_{\|}}{m^{2}}\right)^{2}\right)+ \\
& m_{4} \Delta_{\|} \Delta_{\perp} S_{\perp}
\end{aligned}
$$




$$
\begin{aligned}
\alpha_{\mathrm{rem}}^{2}= & A_{\mathrm{hu}}^{2}+ \\
& \left(\frac{S_{\perp}}{m^{2}}\right)^{2}\left(a_{1}+a_{2}\left(\frac{S_{\|}}{m^{2}}\right)+a_{3}\left(\frac{S_{\|}}{m^{2}}\right)^{2}\right)+ \\
& \left(\frac{\Delta_{\perp}}{m^{2}}\right)^{2}\left(\zeta_{1}+\zeta_{2}\left(\frac{S_{\|}}{m^{2}}\right)+\zeta_{3}\left(\frac{S_{\|}}{m^{2}}\right)^{2}\right)+ \\
& \left(\frac{\Delta_{\|}}{m^{2}}\right)^{2}\left(A_{2}+n_{2}\left(\frac{S_{\|}}{m^{2}}\right)+n_{3}\left(\frac{S_{\|}}{m^{2}}\right)^{2}\right)+ \\
& \left(\frac{S_{\perp} \cdot \hat{n}}{m^{2}}\right)^{2}\left(\mathcal{A}_{1}+\mathcal{A}_{2}\left(\frac{S_{\|}}{m^{2}}\right)+\mathcal{A}_{3}\left(\frac{S_{\|}}{m^{2}}\right)^{2}\right)+ \\
& \left(\frac{\Delta_{\perp} \cdot \hat{l}}{m^{2}}\right)^{2}\left(\tilde{A}_{1}+\tilde{A}_{2}\left(\frac{S_{\|}}{m^{2}}\right)+\tilde{A}_{3}\left(\frac{S_{\|}}{m^{2}}\right)^{2}\right)+ \\
& p_{4} \Delta_{\|} \Delta_{\perp} S_{\perp},,
\end{aligned}
$$

where $\hat{n}$ and $\hat{l}$ are unit vectors in the orbital plane. In general, we do not know the orientation of these two vectors. In our error analysis of the $\mathrm{N}$ configurations above, we assumed the two directions are equal. This introduces an error into our formula which we quantify by comparing the predicted and measured values of $\delta \mathcal{M}$ and $\alpha_{\text {rem }}$. In practice, we suggest taking a fiducial orientation for $\hat{n}=\hat{l}$ and then considering distributions of all possible azimuthal orientations of the initial spin. The resulting distribution of remnants will be independent of the fiducial choice of $\hat{n}$. Note that Eqs. (58) and (59) use the form $\delta \mathcal{M}=E_{c}+E_{\phi} \cos ^{2} \phi$, etc.. To use the primed coefficients, replace terms like $\left(\vec{V}_{\perp} \cdot p\right)^{2}$ with $2\left[\left(\vec{V}_{\perp} \cdot p\right)^{2}-\vec{V}_{\perp}^{2}\right]$. The latter is more accurate only when dropping the $E_{\phi}$ and $A_{\phi}$ terms, otherwise, the two expressions are equivalent.

For reference, we also give the formulas of 60] for $\delta \mathcal{M}_{\mathrm{hu}}$ and $A_{\mathrm{hu}}$ in terms of $S_{\|}$here

$$
\begin{aligned}
\delta \mathcal{M}_{\mathrm{hu}}= & 0.0025829-\frac{0.0773079}{2 \frac{S_{\|}}{m^{2}}-1.693959} \\
A_{\mathrm{hu}}= & 0.68640260+0.3066014\left(\frac{2 S_{\|}}{m^{2}}\right)- \\
& 0.0268433\left(\frac{2 S_{\|}}{m^{2}}\right)^{2}-0.0098019\left(\frac{2 S_{\|}}{m^{2}}\right)^{3}- \\
& 0.0049935\left(\frac{2 S_{\|}}{m^{2}}\right)^{4}
\end{aligned}
$$

To measure the error in our empirical formula, we provide two types of residuals. First, for a given family with constant $\theta$ we compare the measured $E_{c}^{\prime}, E_{\phi}^{\prime}, A_{c}^{\prime}$, and $A_{\phi}^{\prime}$ of that family with the predictions of Eq. (55)-(57), where we use the average value of $S_{\|}, S_{\perp}, \Delta_{\|}$, and $\Delta_{\perp}$ of that family. Second, we measure the residuals of each member of all families using the known values of $\phi$ (which were obtained from the original fits). When calculating these residuals, we also consider the $\mathrm{K}, \mathrm{L}$, and $\mathrm{S}$ families of [17. We show the first type of residual in Table XI and we show the relative errors in $\delta \mathcal{M}$ and $\alpha_{\text {rem }}$ for all 175 configurations in Fig. 19 and Fig. 20 . However, because of a sign ambiguity in the term $\Delta_{\|} \Delta_{\perp} S_{\perp}$, we do not include these terms when calculating the residuals in Figs. 19 and 20. This also means that the N configuration do not contribute to the fitting formulas and can therefore be used to verify the accuracy of the formulas for more generic configurations. We find that the relative errors are within $\pm 2.5 \%$ and that the distribution of errors in $\delta \mathcal{M}$ is wider but more symmetric then the distribution of errors in $\alpha_{\mathrm{rem}}$. As an aside, we note that the large relative errors in the coefficients in $A_{\phi}$ and $E_{\phi}$ only have a small effect on the net error in the predictions. The errors in the coefficients seen in Table XI are a consequence of the smallness of $E_{\phi}$ and $A_{\phi}$ relative to $E_{c}$ and $A_{c}$ (and because we would ideally choose twice as many $\phi$ configurations to accurately fit a $\cos 2 \phi$ dependence).

Our formulas are based on the vectors $\vec{S}$ and $\vec{\Delta}$. As shown in Fig. 21. $S_{\|}$and the magnitude of $\vec{S}_{\perp}$ are conserved (approximately) throughout a simulation. This is consistent with the predictions of PN theory (see Fig. 22). However, while $|\vec{\Delta}|$ is conserved (which is a consequence of $|\vec{\Delta} / 2|^{2}=\left|\vec{S}_{1}\right|^{2}+\left|\vec{S}_{2}\right|^{2}-|\vec{S}|^{2}$ for equal mass binaries), the components $\Delta_{\|}$and $\Delta_{\perp}$ are not (in general). Due to symmetry, the components of $\vec{\Delta}$ are conserved for the SP, HK (due to $\pi$-symmetry the spins cannot rotate with respect to each other), and $\mathrm{N}$ configurations (where $\vec{\Delta}=2 \vec{S}$ ). The question then arises, where should one evaluate $\Delta_{\|}$and $\Delta_{\perp}$. For our runs, this issue only arises for the K, L, and S configurations. Fortunately, this represents 24 separate simulations. Thus we were able to explore this question. We found that using the initial values of $\Delta_{\|}$and $\Delta_{\perp}$ (which was done in Table XI and Fig. 19p reproduced the measured $\delta \mathcal{M}$ and $\alpha_{\text {rem }}$ with sufficient accuracy (i.e. within $2.5 \%$ ).

Finally, we note that while Eq. (59) only provides the magnitude of $\alpha_{\text {rem }}$, we can obtain its direction (to within a few degrees) from the direction of $\vec{J}$ at any point along the binaries evolution.

\section{DISCUSSION}

As an application to cosmological growth of black holes, it is interesting to determine the spin distribution of BHs after many generations of mergers. As our formulas for the final remnant spin have not been fully extended to the unequal mass case, we consider the consequence of the mergers of equal-mass binaries. Here, we neglect the radiation of energy, which would affect the mass-ratio distribution of subsequent generations. We find that the spin distribution relaxes to a universal distribution independent of the initial distribution. We verified this by starting with three different spin distributions: non-spinning BHs, a spin distribution uniform in $[0,1]$, and all BHs with unit spin. We first assume that the spin directions are uniformly distributed on the sphere 


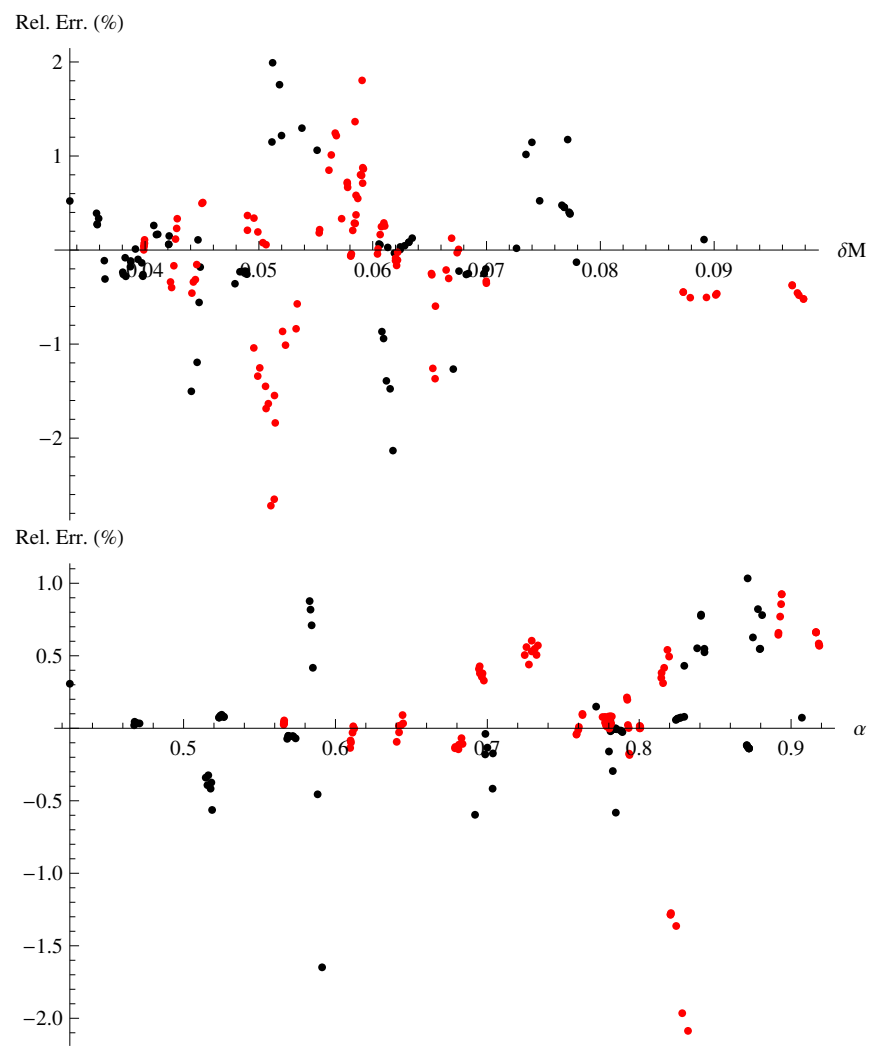

FIG. 19: The relative errors in the predicted values of $\delta \mathcal{M}$ and $\alpha_{\text {rem }}$ in percent for all 175 runs used in the fits and analysis in this paper. Here the black dots correspond to simulations used in the fits and the red dots to independent simulations. Because we drop terms of the form $\Delta_{\|} \Delta_{\perp} S_{\perp}$, the $\mathrm{N}$ configurations are independent runs here.

and then, to model how accretion disks torques may affect our results, we also consider the case where the spins are uniform in the upper hemisphere and uniform in the section of the sphere $\theta<30^{\circ}$. Figures 23 25 show how the spin distribution changes from merger generation to generation. The effect of spin alignment is more clearly shown in Fig. 26, which shows all three final spin distributions. Using these universal spin distributions, we find the distributions of radiated energy, also shown in Fig. 26 .

Note that our distributions of the magnitude of the spin are consistent with the observed spins in 19 active galactic nuclei (See Fig. 6 in Ref. [65]), as most of the observed values lie in the high spin region.

While all our simulations have assumed equal-mass binaries (we will study explicitly the unequal-mass case in a following paper), the key aspect of the dynamics of the binary that allows us to model the direction of the remnant spin, i.e. simple precession, occurs for comparablemass binaries, as well. In order to estimate the critical mass ratio $q=m_{1} / m_{2}=q_{\text {critical }}$ below which it is possible to have transitional precession (which can reverse the direction of the total angular momentum of the binary system), we will consider the maximally spinning black-

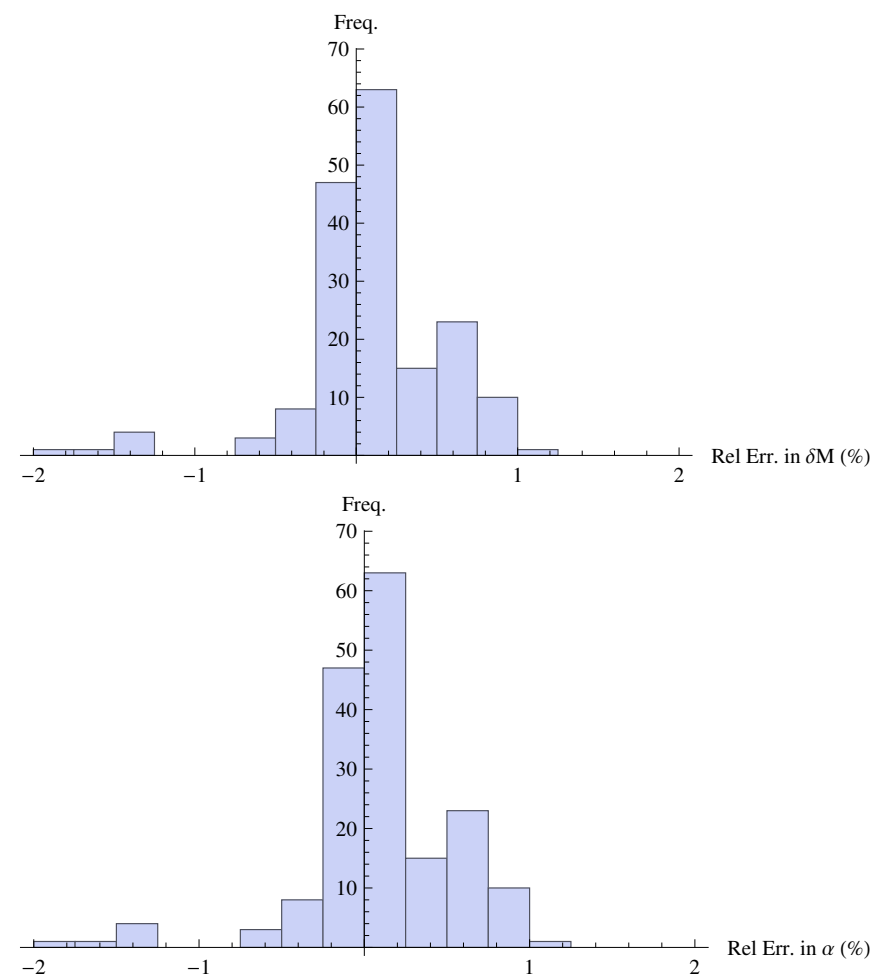

FIG. 20: Histograms of the relative errors in Fig. 19. The $x$ axis is relative error in percent and the bin width is $0.25 \%$.

hole binaries where the spins are counteraligned with the orbital angular momentum $\vec{L}$. Here, at the transition point, $J_{\|}$changes from positive to negative and net angular momentum rotates by $\pi$ radians. A good estimate for when this transition occurs can be obtained using the 3.5PN expression for $\vec{L}$ for quasicircular orbits (see Eq. (4.7) of Ref. [66]).

$$
\begin{aligned}
\frac{L}{m^{2}}= & \nu\left(1+\left(\frac{3}{2}+\frac{1}{6} \nu\right) x+\left(-\frac{35}{6} s-\frac{5}{2} d m \Delta\right) x^{3 / 2}+\right. \\
& \left(\frac{27}{8}-\frac{19}{8} \nu+\frac{1}{24} \nu^{2}\right) x^{2}+ \\
& \left(\left(-\frac{77}{8}+\frac{427}{72} \nu\right) s+\left(-\frac{21}{8}+\frac{35}{12} \nu\right) d m \Delta\right) x^{5 / 2}+ \\
& \left(\frac{135}{16}+\left(-\frac{6889}{144}+\frac{41}{24} \pi\right) \nu+\frac{31}{24} \nu^{2}+\frac{7 \nu^{3}}{1296}\right) x^{3}+ \\
& \left(\left(-\frac{405}{16}+\frac{1101}{16} \nu-\frac{29}{16} \nu^{2}\right) s+\right. \\
& \left.\left.\left(-\frac{81}{16}+\frac{117}{4} \nu-\frac{15}{16} \nu^{2}\right) d m \Delta\right) x^{7 / 2}\right) \frac{1}{\sqrt{x}}
\end{aligned}
$$

where $x=\left(m \Omega_{\text {orbital }}\right)^{2 / 3}, \nu=q /(1+q)^{2}$, and for our maximally spinning counteraligned configurations $s=$ $-\left(1+q^{2}\right) /(1+q)^{2}$ and $d m \Delta=-(1-q)^{2} /(1+q)^{2}$. Assuming $S$ is conserved, we find the critical value $q_{\text {critical }}$ such that, for a given frequency $\Omega_{\text {orbit }}, J$ vanishes. We plot $q_{\text {critical }}$ versus $\Omega_{\text {orbit }}$ in Fig. 27. The plot covers the 

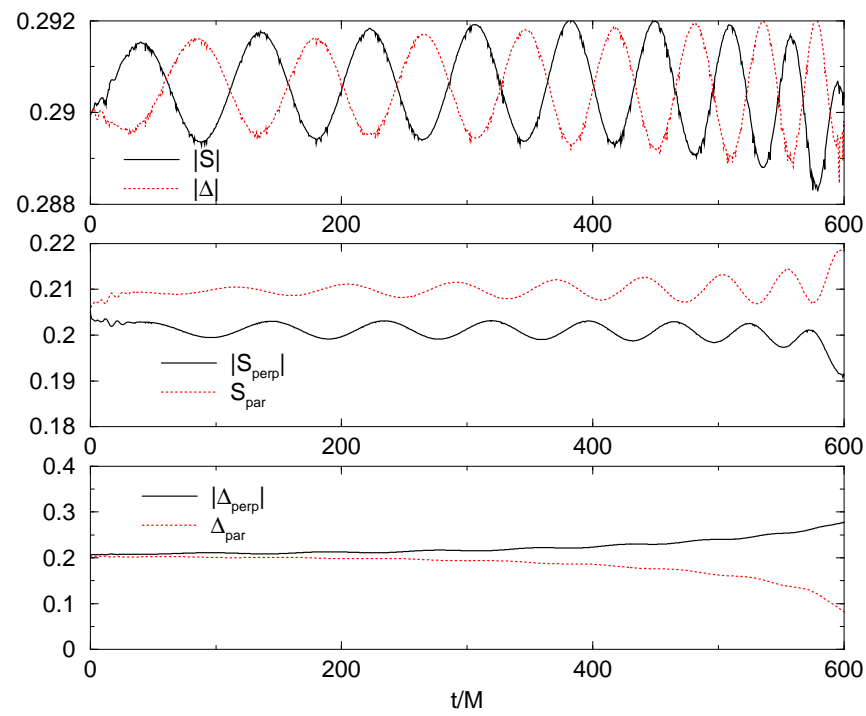

FIG. 21: The magnitudes of $\vec{S}$ and $\vec{\Delta}$ (top panel), the magnitudes of the components of $\vec{S}$ (middle panel), and the components of $\vec{\Delta}$ (lower panel) for a typical L configuration (one spin initially in the plane, one spin perpendicular to the plane). The magnitude of $\vec{S}$ and $\vec{\Delta}$ are both conserved to a high degree (note the different scales). The components of $\vec{S}$ are also conserved, but not to the same degree as $|\vec{S}|$ itself. Finally, the components of $\vec{\Delta}$ are not conserved.

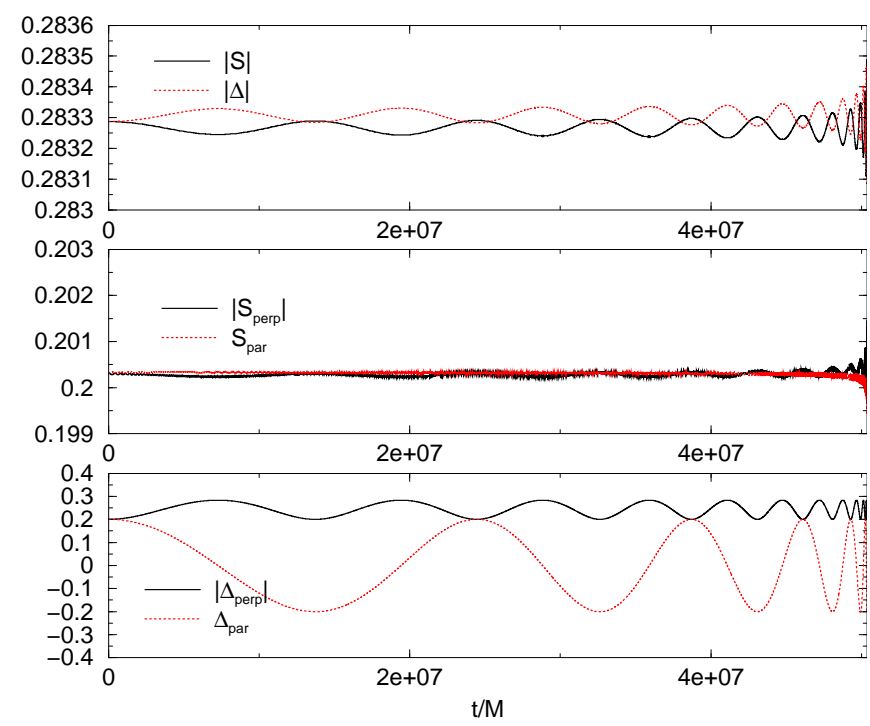

FIG. 22: The magnitudes of $\vec{S}$ and $\vec{\Delta}$ (top panel), the magnitudes of the components of $\vec{S}$ (middle panel), and the components of $\vec{\Delta}$ (lower panel) for a $3.5 \mathrm{PN}$ evolution of a typical L configuration (one spin initially in the plane, one spin perpendicular to the plane) starting from an initial quasicircular orbit at a separation of $\sim 158 M$. The magnitude of $\vec{S}$ and $\vec{\Delta}$ are both conserved to a high degree (note the different scales). The components of $\vec{S}$ are also conserved, but not to the same degree as $|\vec{S}|$ itself. Finally, the components of $\vec{\Delta}$ are not conserved.

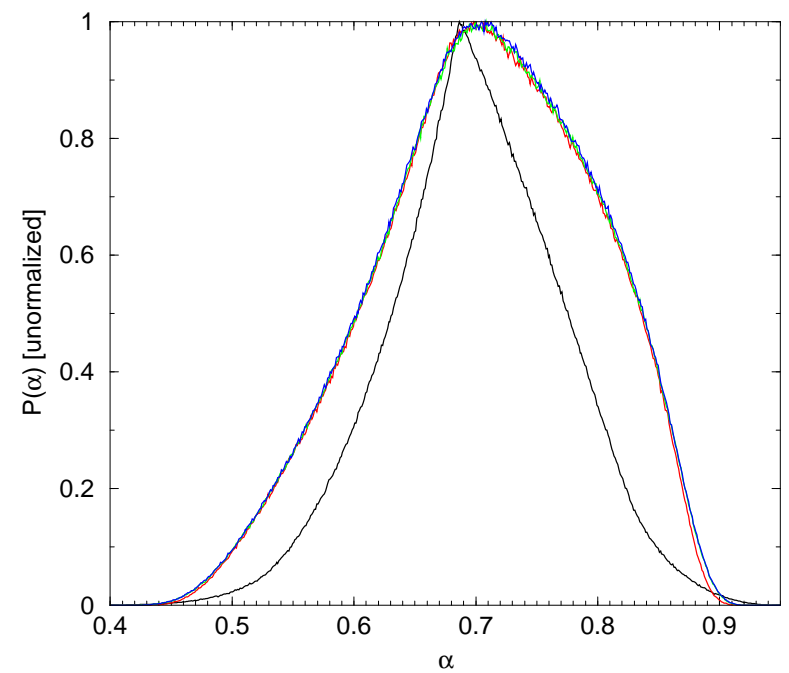

FIG. 23: The approach to a universal spin distribution after a few mergers when the BH spins are assumed to be uniform on the entire sphere $\theta<180^{\circ}$. The wide black curve corresponds to the first generation. The wide red curve corresponds to the second generation. The remaining generations are green (3rd), and blue (4th).

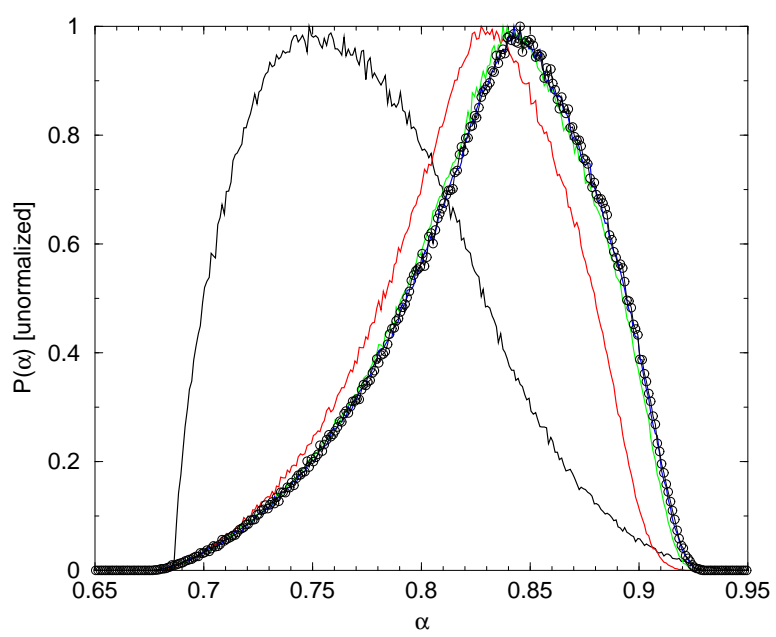

FIG. 24: The approach to a universal spin distribution after a few mergers when the $\mathrm{BH}$ spins are assumed to be uniform on the section of the sphere $\theta<90^{\circ}$. The wide black curve corresponds to the first generation. The wide red curve corresponds to the second generation. The remaining generations are green (3rd), blue (4th), and black circles (5th).

inspiral regime down to the plunge. Note that transitional precession does not occur if $q \gtrsim 1 / 4$. This is in good agreement with our previous estimate 21, which was based on considering the complementary regime of a point particle moving in a Kerr background.

Ultimately, in order to determine the mass ratio dependence of the remnant mass and spin, one has to perform a systematic study of binaries in the regime $1 / 10 \leq q \leq 1$ and fit the remaining coefficients of the terms in Tables 


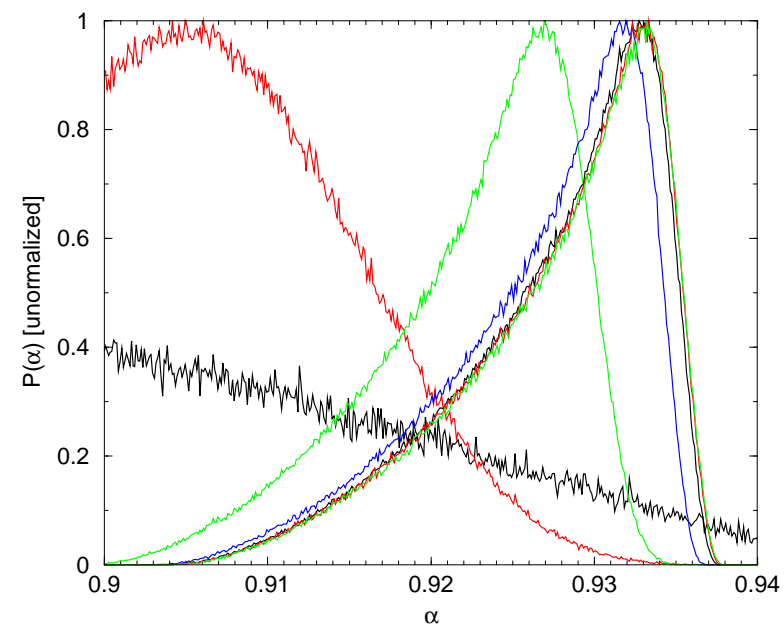

FIG. 25: The approach to a universal spin distribution after a few mergers when the $\mathrm{BH}$ spins are assumed to be uniform on the section of the sphere $\theta<30^{\circ}$. The wide black curve corresponds to the first generation. The wide red curve corresponds to the second generation. The remaining generations are green (3rd), blue (4th), followed by the narrow black (5th), red (6th), and green(7th) curves.

VHVI (which explicitly depend on $\delta m$ ). This is currently under investigation by the authors and will provide a unified phenomenological description for the remnant mass, spin and recoil velocities [62].

\section{CONCLUSION}

We developed a set of generic empirical formulas to predict the final remnant mass and spin from the merger of two black holes. In order to obtain these empirical formulas, we extended our recent framework for modeling the remnant recoil, which uses a combination of $\mathrm{PN}$-inspired variables, fitting parameters, and symmetry arguments [17. This formalism for modeling remnant properties with arbitrary mass ratios and spins is summarized in Tables V V VI, Note that we used the same symbolic expansion (39)- 40 for both the radiated energy and the magnitude of the remnant spin. We justify our assumption that a decomposition of the spins in terms of components parallel to, and perpendicular to, the orbital angular momentum is appropriate in light of the results in Fig. 7, which shows how well of $\vec{S} \cdot \vec{L}$ is conserved, and those of Ref. 21] were the final mass and spins can be approximated by the nonprecessing hangup formula. We also assume that, given the results in Fig. 4. which shows how well the direction of the total angular momentum is conserved, the final spin direction agrees with that of the total angular momentum at large binary separations.

We performed 43 new simulations and combined these results with over 130 other simulations from Refs. [17, 34 to fit all relevant equal-mass coefficients in our phe-
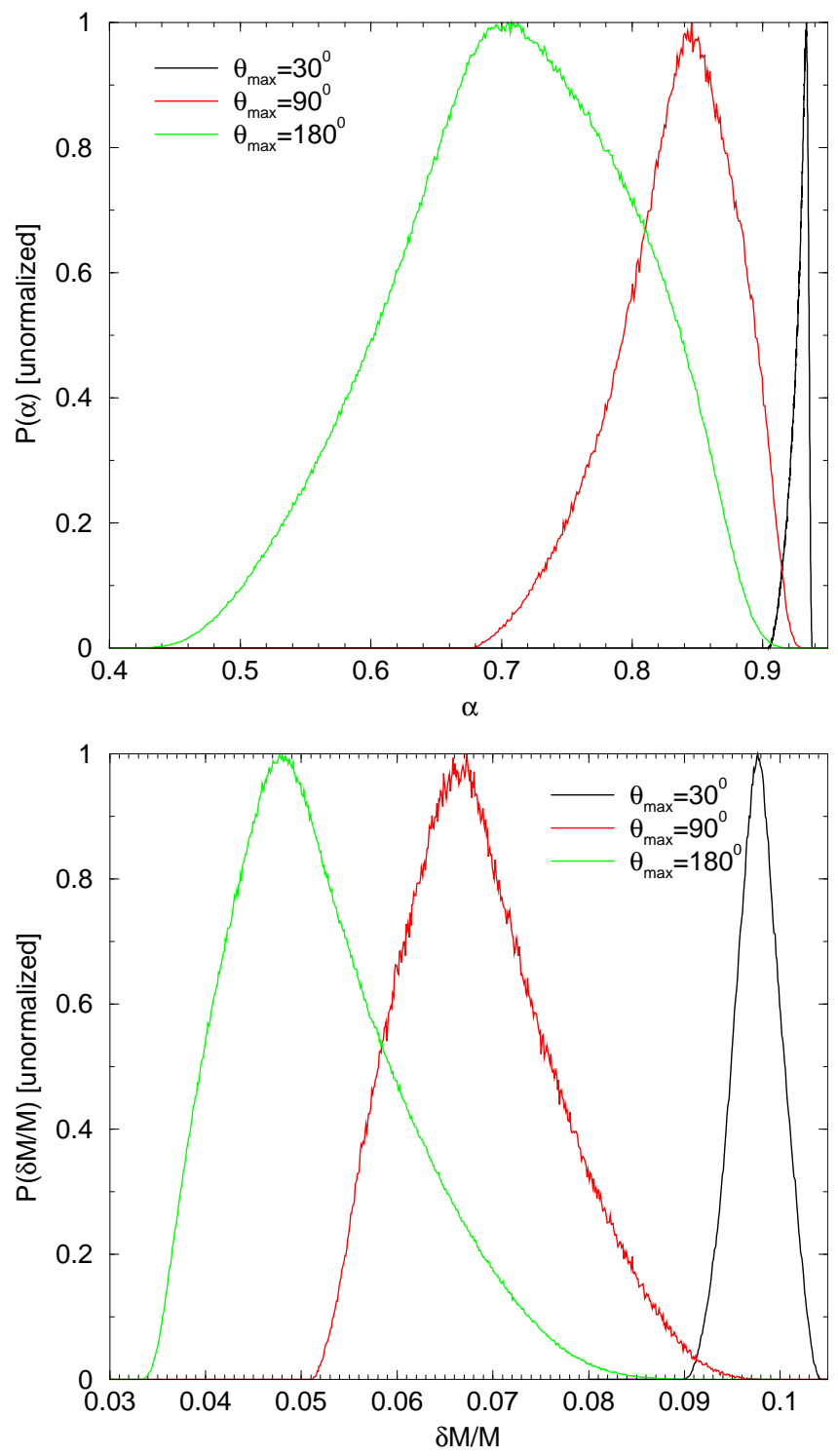

FIG. 26: The final distributions of spin magnitudes and radiated mass for the remnants of BHB mergers. The angular distributions are assumed to be uniform on the following segments of the sphere $\theta<180^{\circ}, \theta<90^{\circ}$, and $\theta<30^{\circ}$.

nomenological formulas (39)-(40). This large set of similarly constructed (initial orbital frequencies, number of orbits, total run times) runs for equal-mass binaries allowed us to obtain the coefficients for the most relevant nonlinear terms in our expansion formulas for the radiated mass and remnant spin to a consistent accuracy. We estimate the relative errors lie within $2.5 \%$. The natural next step is to extend these formulas to unequal-mass, generic-spin, BHB mergers. A comparatively large number of such generic simulations is needed for this task.

Finally, we note that our results lend additional support for the use of non-precessing waveforms in the modeling of precessing waveforms [27, 67, 68. That procedure (see Ref. 20] for a recent review) uses hangup 


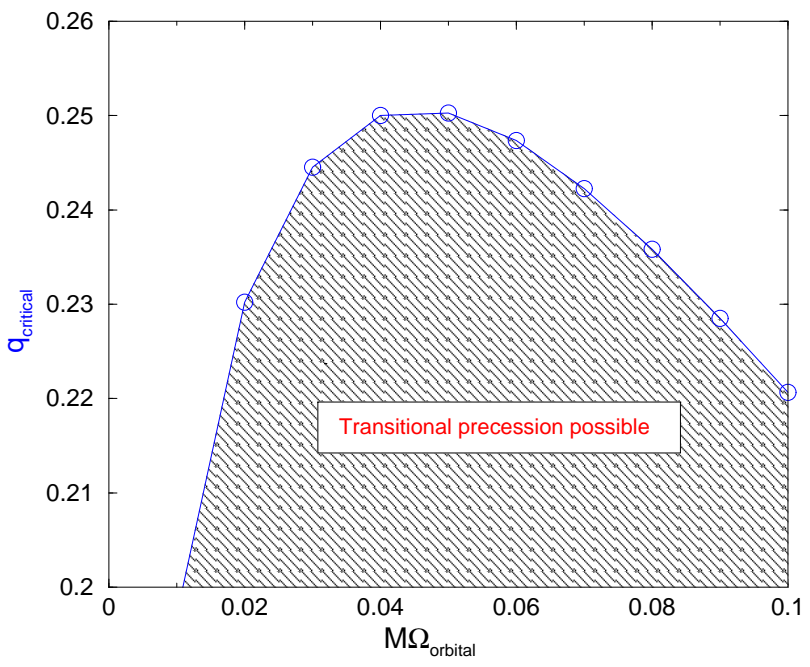

FIG. 27: Critical mass ratio for counteraligned, maximally spinning black holes according to 3.5PN. Transition only can occur in the parameter region below this curve.

waveforms (i.e. non-precessing, spinning waveforms) in a dynamically rotating frame to model the waveform from precessing binaries. Our results show that the component of $\vec{S}$ along $\vec{L}$ is conserved, which is the only component of the spin in the hangup case and thus provides a unique hangup waveform as a starting point for the modeling. If the Bondi News $N(t)$ for a precessing waveform can be described by a time dependent ro- tation $\mathbf{R}(t)$ acting on a non-precessing waveform $N_{\mathrm{np}}$, then $\bar{N} N=\overline{\mathbf{R}(t) N_{\mathrm{np}}} \mathbf{R}(t) N_{\mathrm{np}}=\bar{N}_{\mathrm{np}} N_{\mathrm{np}}$ (where the bar denoted complex conjugation) and the total radiated energy for the precessing waveforms is equal to that of the hangup waveform. Again, we see that the energy radiated by the binary matches, to within an accuracy of $\lesssim 10 \%$, the energy radiated from the equivalent hangup waveform. The fact that we see this agreement for a broad class of binaries is suggestive. Our results will also be important for modeling the late time waveform because the plunge and ringdown part of the waveforms should have the characteristic frequency of the actual spin of the merger remnant, $\alpha_{\mathrm{rem}}^{2} \approx \alpha_{\mathrm{np}}^{2}+\alpha_{\perp}^{2}$, and thus deviate from the original hangup waveform.

\section{Acknowledgments}

The authors wish to thank the referee for a very careful reading of the original manuscript and for many useful suggestions for improving it. The authors gratefully acknowledge the NSF for financial support from Grants PHY-1305730, PHY-1212426, PHY1229173, AST-1028087, PHY-0969855, OCI-0832606, and DRL-1136221. Computational resources were provided by XSEDE allocation TG-PHY060027N, and by NewHorizons and BlueSky Clusters at Rochester Institute of Technology, which were supported by NSF grant No. PHY-0722703, DMS-0820923, AST-1028087, and PHY-1229173.
[1] F. Pretorius, Phys. Rev. Lett. 95, 121101 (2005), grqc/0507014.

[2] M. Campanelli, C. O. Lousto, P. Marronetti, and Y. Zlochower, Phys. Rev. Lett. 96, 111101 (2006), grqc/0511048.

[3] J. G. Baker, J. Centrella, D.-I. Choi, M. Koppitz, and J. van Meter, Phys. Rev. Lett. 96, 111102 (2006), grqc/0511103.

[4] L. Blanchet, Living Rev. Rel. 5, 3 (2002), gr-qc/0202016.

[5] J. Baker, M. Campanelli, and C. O. Lousto, Phys. Rev. D 65, 044001 (2002), gr-qc/0104063.

[6] C. O. Lousto and Y. Zlochower, Phys. Rev. Lett. 106, 041101 (2011), 1009.0292.

[7] U. Sperhake, V. Cardoso, C. D. Ott, E. Schnetter, and H. Witek, Phys. Rev. D84, 084038 (2011), 1105.5391.

[8] G. Lovelace, M. Boyle, M. A. Scheel, and B. Szilagyi, Class. Quant. Grav. 29, 045003 (2012), 1110.2229.

[9] C. O. Lousto and Y. Zlochower, Phys. Rev. D88, 024001 (2013), 1304.3937.

[10] M. Campanelli, C. O. Lousto, and Y. Zlochower, Phys. Rev. D74, 041501(R) (2006), gr-qc/0604012.

[11] M. Campanelli, C. O. Lousto, Y. Zlochower, and D. Merritt, Phys. Rev. Lett. 98, 231102 (2007), gr-qc/0702133.

[12] J. A. González, M. D. Hannam, U. Sperhake, B. Brugmann, and S. Husa, Phys. Rev. Lett. 98, 231101 (2007), gr-qc/0702052.
[13] J. Healy et al., Phys. Rev. Lett. 102, 041101 (2009), 0807.3292 .

[14] C. O. Lousto and Y. Zlochower, Phys. Rev. Lett. 107, 231102 (2011), 1108.2009.

[15] M. Campanelli, C. O. Lousto, Y. Zlochower, and D. Merritt, Astrophys. J. 659, L5 (2007), gr-qc/0701164.

[16] M. Campanelli, C. O. Lousto, H. Nakano, and Y. Zlochower, Phys. Rev. D79, 084010 (2009), 0808.0713.

[17] C. O. Lousto and Y. Zlochower, Phys. Rev. D87, 084027 (2013), 1211.7099.

[18] A. H. Mroue, M. A. Scheel, B. Szilagyi, H. P. Pfeiffer, M. Boyle, et al., Phys.Rev.Lett. 111, 241104 (2013), 1304.6077.

[19] I. Hinder et al. (Perimeter Institute for Theoretical Physics), Class. Quant. Grav. 31, 025012 (2014), 1307.5307.

[20] M. Hannam (2013), 1312.3641.

[21] C. O. Lousto and Y. Zlochower, Phys.Rev. D89, 021501 (2014), 1307.6237.

[22] T. A. Apostolatos, C. Cutler, G. J. Sussman, and K. S. Thorne, Phys. Rev. D 49, 6274 (1994).

[23] E. Racine, Phys. Rev. D78, 044021 (2008), 0803.1820.

[24] E. Barausse and L. Rezzolla, Astrophys. J. Lett. 704, L40 (2009), 0904.2577.

[25] L. Boyle, M. Kesden, and S. Nissanke, Phys. Rev. Lett. 100, 151101 (2008), 0709.0299. 
[26] L. Boyle and M. Kesden, Phys. Rev. D78, 024017 (2008), 0712.2819 .

[27] M. Hannam, P. Schmidt, A. Boh, L. Haegel, S. Husa, et al. (2013), 1308.3271.

[28] M. Volonteri, M. Sikora, J.-P. Lasota, and A. Merloni, Astrophys.J. 775, 94 (2013), 1210.1025.

[29] P. Amaro-Seoane, S. Konstantinidis, M. D. Freitag, M. C. Miller, and F. A. Rasio (2012), 1211.6738.

[30] C. O. Lousto, M. Campanelli, Y. Zlochower, and H. Nakano, Class. Quant. Grav. 27, 114006 (2010), 0904.3541.

[31] A. Buonanno, L. E. Kidder, and L. Lehner, Phys. Rev. D77, 026004 (2008), arXiv:0709.3839 [astro-ph].

[32] W. Tichy and P. Marronetti, Phys. Rev. D78, 081501 (2008), 0807.2985.

[33] E. Barausse, V. Morozova, and L. Rezzolla, Astrophys. J. 758, 63 (2012), 1206.3803.

[34] C. O. Lousto, Y. Zlochower, M. Dotti, and M. Volonteri, Phys. Rev. D85, 084015 (2012), 1201.1923.

[35] C. O. Lousto, H. Nakano, Y. Zlochower, and M. Campanelli, Phys. Rev. D81, 084023 (2010), 0910.3197.

[36] T. Bogdanovic, C. S. Reynolds, and M. C. Miller, Astrophys. J. 661, L147 (2007), astro-ph/0703054.

[37] M. Dotti, M. Colpi, S. Pallini, A. Perego, and M. Volonteri, Astrophys. J. 762, 68 (2013), 1211.4871.

[38] M. Campanelli, C. O. Lousto, Y. Zlochower, B. Krishnan, and D. Merritt, Phys. Rev. D75, 064030 (2007), gr-qc/0612076.

[39] L. E. Kidder, Phys. Rev. D52, 821 (1995), grqc/9506022.

[40] Cactus Computational Toolkit home page: http://cactuscode.org.

[41] Einstein Toolkit home page: http://einsteintoolkit.org.

[42] E. Schnetter, S. H. Hawley, and I. Hawke, Class. Quant. Grav. 21, 1465 (2004), gr-qc/0310042.

[43] M. Ansorg, B. Brügmann, and W. Tichy, Phys. Rev. D70, 064011 (2004), gr-qc/0404056.

[44] S. Brandt and B. Brügmann, Phys. Rev. Lett. 78, 3606 (1997), gr-qc/9703066.

[45] Y. Zlochower, J. G. Baker, M. Campanelli, and C. O. Lousto, Phys. Rev. D72, 024021 (2005), gr-qc/0505055.

[46] P. Marronetti, W. Tichy, B. Brügmann, J. Gonzalez, and U. Sperhake, Phys. Rev. D77, 064010 (2008), 0709.2160.

[47] C. O. Lousto and Y. Zlochower, Phys. Rev. D77, 024034 (2008), 0711.1165.

[48] J. Thornburg, Class. Quant. Grav. 21, 743 (2004), gr- qc/0306056.

[49] O. Dreyer, B. Krishnan, D. Shoemaker, and E. Schnetter, Phys. Rev. D67, 024018 (2003), gr-qc/0206008.

[50] F. Herrmann, I. Hinder, D. Shoemaker, P. Laguna, and R. A. Matzner, Astrophys. J. 661, 430 (2007), grqc/0701143.

[51] L. Rezzolla et al., Astrophys. J. 679, 1422 (2008), arXiv:0708.3999 [gr-qc].

[52] J. Winicour, in General Relativity and Gravitation Vol 2, edited by A. Held (Plenum, New York, 1980), pp. 71-96.

[53] C. O. Lousto and Y. Zlochower, Phys. Rev. D76, 041502(R) (2007), gr-qc/0703061.

[54] M. Campanelli and C. O. Lousto, Phys. Rev. D59, 124022 (1999), gr-qc/9811019.

[55] B. Krishnan, C. O. Lousto, and Y. Zlochower, Phys. Rev. D76, 081501 (2007), 0707.0876.

[56] R. O'Shaughnessy, J. Healy, L. London, Z. Meeks, and D. Shoemaker, Phys. Rev. D85, 084003 (2012), 1201.2113.

[57] M. Boyle, Phys.Rev. D87, 104006 (2013), 1302.2919.

[58] M. Ruiz, R. Takahashi, M. Alcubierre, and D. Nunez, Gen. Rel. Grav. 40, 2467 (2008), 0707.4654.

[59] E. Racine, A. Buonanno, and L. E. Kidder, Phys. Rev. D80, 044010 (2009), 0812.4413.

[60] D. A. Hemberger, G. Lovelace, T. J. Loredo, L. E. Kidder, M. A. Scheel, et al., Phys.Rev. D88, 064014 (2013), 1305.5991.

[61] T. Damour, Phys. Rev. D64, 124013 (2001), grqc/0103018.

[62] C. O. Lousto and Y. Zlochower (2014), in preparation.

[63] G. B. Arfken and H. J. Weber, Mathematical methods for physicists 6th ed. (2005).

[64] C. O. Lousto and Y. Zlochower, Phys. Rev. D79, 064018 (2009), 0805.0159.

[65] C. S. Reynolds, Class.Quant.Grav. 30, 244004 (2013), 1307.3246.

[66] A. Bohe, S. Marsat, G. Faye, and L. Blanchet, Class. Quant. Grav. 30, 075017 (2013), 1212.5520.

[67] M. Boyle, R. Owen, and H. P. Pfeiffer, Phys.Rev. D84, 124011 (2011), 1110.2965.

[68] L. Pekowsky, R. O'Shaughnessy, J. Healy, and D. Shoemaker, Phys.Rev. D88, 024040 (2013), 1304.3176.

[69] We use the conventions of [57] to define $\mathbf{J}$ which differ from the conventions of 58 by a factor of $-i$.

\section{Appendix A: Results from the SP and UD families of configurations}

In this appendix, we give the initial data parameters for all SP and UD configurations in Table XII the energy and angular momentum radiated in Table XIII (as measured by directly from $\psi_{4}$ and inferred from the remnant mass and spin obtained using the isolated horizon formalism), the radiated and remnant angular momentum and the angle between the initial and final angular momentum in Table XIV the final remnant spin and mass loss (starting from infinite separation) in Table XIII, and finally, the measured recoils (calculated using $\ell \leq 4$ modes) for the UD configurations in Table XVI. Note that we approximate the mass loss from infinity using $\delta \mathcal{M}=\left(M_{1}+M_{2}-\right.$ $\left.M_{\text {rem }}\right) /\left(M_{1}+M_{2}\right)$, where $M_{\text {rem }}$ is the remnant BH's mass and $M_{1}$ and $M_{2}$ are the initial masses of the two BHs in the binary (which is a good approximation for their masses when the binary was infinitely separated.

Here we also compare the performance of using $\vec{L}(t)=\vec{J}(t)-\vec{S}(t)$ and $\hat{S}_{\text {coord }}$ to define the component of the spin $S_{\|}$. In Fig. 28 , we show the angles $\{\vec{L}, \vec{S}\}$ and $\left\{\vec{L}_{\text {coord }}, \vec{S}\right\}$ versus time for all the N configurations (which are more 


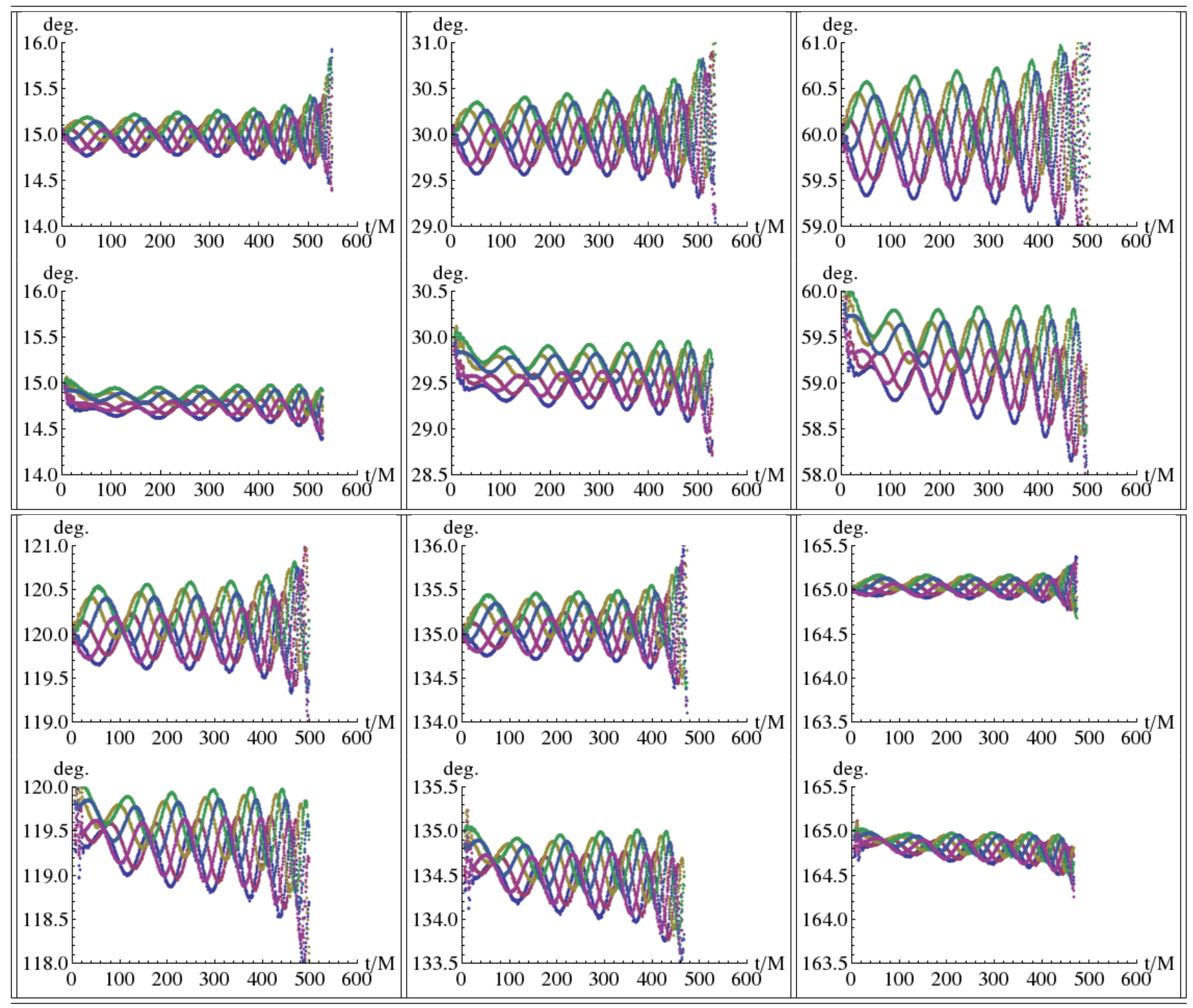

FIG. 28: The angles $\{\vec{L}, \vec{S}\}$ and $\left\{\vec{L}_{\text {coord }}, \vec{S}\right\}$ versus time for all the N configurations. The panels are ordered from left to right and top to bottom with the top-left corresponding the NTH15 and the bottom right to NTH165. For each panel, the top plot shows $\{\vec{L}, \vec{S}\}$ and the bottom shows $\left\{\vec{L}_{\text {coord }}, \vec{S}\right\}$. Note the small secular trends in $\left\{\vec{L}_{\text {coord }}, \vec{S}\right\}$ (here a small net negative slope) that are not present in $\{\vec{L}, \vec{S}\}$. Note, however, that the amplitude of sinusoidal oscillations are slightly larger for $\{\vec{L}, \vec{S}\}$.

general than the SP configurations). A slight secular trend is apparent in $\left\{\vec{L}_{\text {coord }}, \vec{S}\right\}$ that seems to be suppressed in $\{\vec{L}, \vec{S}\}$. 
TABLE XII: Initial data parameters. The BHs have initial position $\vec{r}=( \pm x, 0,0)$, momenta $\left(0, \pm p_{y}, 0\right)$, spins $\pm\left(S_{x}, S_{y}, S_{z}\right)$, and puncture mass parameters $m_{p}$, as well as the ADM mass $M_{\mathrm{ADM}}$, ADM angular momentum $\left(J_{x}, J_{y}, J_{z}\right)$, initial eccentricity $e_{i n i}$, eccentricity just prior to plunge $e_{f i n}$, and total number of orbits $N$. Masses, positions, and momenta are given in units of an arbitrary mass $M$, while angular momenta are given in units of $M^{2}$.

\begin{tabular}{|c|c|c|c|c|c|c|c|c|c|c|c|}
\hline Conf & $m_{p}$ & $x$ & $p_{y}$ & $S_{x}$ & $S_{y}$ & $S_{z}$ & $M_{\mathrm{ADM}}$ & $J_{x}$ & $J_{y}$ & $J_{z}$ & $N_{e_{i n i}}^{e_{f i n}}$ \\
\hline SPTH0PH0 & 0.30334 & 4.10893 & 0.10524 & 0.00000 & 0.00000 & 0.20534 & 1.00000 & 0.00000 & 0.00000 & 1.27551 & \\
\hline SPTH48PH0 & .30285 & 4.16101 & .10656 & 0.00000 & 0.15294 & 0.13679 & 1.00000 & 0.00000 & 0.30588 & 1.16042 & $5_{0.004}^{0.02}$ \\
\hline SPTH48PH30 & .30293 & 4.16101 & .10656 & -0.07647 & 0.13245 & 0.13679 & 1.00000 & -0.15294 & 0.26490 & 1.16042 & \\
\hline SPTH48PH60 & 30309 & 4.16101 & 10656 & -0.13245 & 0.07647 & 13679 & 1.00000 & -0.26490 & 0.15294 & 1.16042 & \\
\hline SPTH48PH90 & 0317 & 4.16101 & 0656 & -0.15294 & 0.00000 & 13679 & 1.00000 & -0.30588 & 0.00000 & 1.16042 & \\
\hline SPTH48PH120 & 0.30309 & 4.16101 & .10656 & -0.13245 & -0.07647 & 0.13679 & 1.00000 & -0.26490 & -0.15294 & 1.16042 & \\
\hline SPTH48PH150 & 0.30293 & 4.16101 & .10656 & -0.07647 & -0.13245 & 0.13679 & 1.00000 & -0.15294 & -0.26490 & 1.16042 & \\
\hline SPTH70PH0 & 0.30252 & 4.21471 & 10787 & 0.00000 & 331 & 6835 & 1.00000 & 0.00000 & 0.38662 & 1.04601 & $4.5_{0.005}^{0.02}$ \\
\hline SPTH70PH30 & 30266 & 4.21471 & 10787 & -0.09666 & .16741 & 35 & 1.00000 & -0.19331 & 0.33 & 1.04601 & \\
\hline SPTH70PH60 & 30294 & 4.21471 & 87 & -0.16 & 0.09 & 35 & 0000 & -0.33 & 0.19 & 1.04601 & \\
\hline SPTH70PH90 & 30307 & 4.21471 & 10787 & -0.1 & 0.00 & & 000 & 662 & 000 & 1.04601 & \\
\hline SPTH7C & 294 & 4.21471 & 787 & 741 & -0.09666 & & 0000 & & -0.19331 & 1.04601 & \\
\hline SPTH70PH150 & 0.30266 & 4.21471 & .10787 & -0.09666 & -0.16741 & 0.06835 & 1.00000 & -0.19331 & -0.33482 & 1.04601 & \\
\hline SPTH90PH0 & .30238 & 4.26949 & 917 & 00 & 488 & & & 00 & 0.40977 & 0.93218 & $4_{0.007}^{0.02}$ \\
\hline SPTH90 & 254 & 4.269 & & 0244 & & & & & & 218 & \\
\hline SPTH90PH60 & 0.30287 & 4.26949 & 10917 & -0.17744 & 0.10244 & 00000 & 0000 & 5487 & 0.20488 & 0.93218 & \\
\hline SPTH90РН90 & 30303 & 4.26949 & .10917 & -0.20488 & 0.00000 & 0.00000 & 1.00000 & -0.40977 & 0.00000 & 218 & \\
\hline SPTH9 & 287 & 4.26 & & -0 . & 244 & & & & 488 & & \\
\hline SPTH90PH150 & 254 & 4.26949 & 917 & -0.10244 & -0.17744 & 0000 & 000 & 488 & -0.35487 & 0.93218 & \\
\hline SPTH110PH0 & 30396 & 5.06743 & 9829 & 0.00000 & 0.19250 & -0.06806 & 1.00000 & 0.00000 & 0.38501 & 0.85999 & $5_{0.007}^{0.02}$ \\
\hline SPTH110PH30 & 410 & 43 & & 625 & & 806 & & & & & \\
\hline SPTH1 & 437 & & & 671 & & 806 & & 342 & & & \\
\hline SPTH110PH90 & 0450 & 5.06743 & 829 & 250 & & 806 & 000 & & & & \\
\hline SPTH110PH120 & 0.30437 & 743 & & & & & & & -0.19250 & & \\
\hline SPTH110PH150 & 0.30410 & 5.06743 & 0.09829 & -0.09625 & -0.16671 & -0.06806 & 1.00000 & -0.19250 & -0.33342 & 0.85999 & \\
\hline SPTH132PH0 & 0.30481 & 5.53592 & 0.09375 & 0.00000 & 0.15193 & -0.13589 & 1.00000 & 0.00000 & 0.30386 & 0.76618 & $5_{0.01}^{0.025}$ \\
\hline SPTH132PH30 & .30490 & 5.53592 & & -0.07596 & & & & -0.15193 & & & \\
\hline SPTH132PH60 & .30506 & 5.53592 & & -0.13157 & & & 1.00000 & & & & \\
\hline SPTH1 & .30514 & 592 & 375 & 193 & & & & 386 & & & \\
\hline SPTH132PH120 & 0.30506 & 5.53592 & 0.09375 & -0.13157 & -0.07596 & 13589 & 0000 & -0.26315 & -0.15193 & 0.76618 & \\
\hline SPTH13 & 0.30490 & 5.53592 & 9375 & -0.07596 & -0.13157 & 589 & 1.00000 & 193 & -0.26315 & 0.76618 & \\
\hline SPTH146PH0 & 559 & 6.07112 & 3839 & 0.00000 & 551 & 6961 & 0000 & 000 & 0.22501 & 0.73403 & \\
\hline SPTH146PH30 & 0.30564 & 6.07112 & & -0.05625 & & 16961 & 1.00000 & -0.11251 & 0.19487 & 0.73403 & \\
\hline SPTH146PH60 & 0.30572 & 6.07112 & & -0.09743 & 0.05625 & 16961 & 1.00000 & & 0.11251 & & \\
\hline SPTH146PH90 & 0.30576 & 6.07112 & 8839 & -0.11251 & 0.00000 & 16961 & 1.00000 & -0.22501 & 0.00000 & 0.73403 & \\
\hline SPTH146PH120 & 0.30572 & 6.07112 & & -0.09743 & -0.05625 & -0.16961 & 1.00000 & -0.19487 & -0.11251 & 0.73403 & \\
\hline SPTH146PH150 & 0.30564 & 6.07112 & 0.08839 & -0.05625 & -0.09743 & -0.16961 & 1.00000 & -0.11251 & -0.19487 & 0.73403 & \\
\hline SPTH180PH0 & 0.30618 & 6.50419 & 0.08473 & 0.00000 & 0.00000 & -0.20332 & 1.00000 & 0.00000 & 0.00000 & 0.69562 & $6.5_{0.005}^{0.025}$ \\
\hline UD0.00 & 0.48965 & 4.50114 & 0.10560 & 0.00000 & 0.00000 & 0.00000 & 1.00000 & 0.00000 & 0.00000 & 0.95064 & $4.5_{0.004}^{0.025}$ \\
\hline UD0.60 & 0.40554 & 4.45038 & 0.10558 & 0.00000 & 0.00000 & 0.15348 & 1.00000 & 0.00000 & 0.00000 & 0.94899 & $4.5_{0.004}^{0.025}$ \\
\hline UD0.70 & 0.36491 & 4.44055 & & 0.00000 & & & & 0.00000 & 0.00000 & & \\
\hline UD0.80 & 0.30366 & 4.43032 & 0.10557 & 0.00000 & 0.00000 & & & & & 0.94769 & $4.5_{0.004}^{0.025}$ \\
\hline UD0.85 & 0.25679 & 4.42506 & 0.10557 & 0.00000 & 0.00000 & 0.21744 & 1.00000 & 0.00000 & 0.00000 & 0.94731 & $4.5_{0.004}^{0.025}$ \\
\hline
\end{tabular}


TABLE XIII: The radiated energy (in units of $M$ ) and angular momentum (in units of $M^{2}$ ) as computed directly from the waveform and by taking the differences between the ADM energy-momentum and the final remnant mass and spin. Here "var." is the expected magnitude of the difference between the two measures assuming the no systematic errors due to finite difference effects. Note how the actual variation between the isolated horizon and radiation measures is about an order of magnitude larger.

\begin{tabular}{|c|c|c|c|c|c|c|c|c|c|c|c|c|}
\hline Conf & $E^{\mathrm{rad}}$ & $\delta E^{\mathrm{ih}}$ & AI. & $\delta J_{x}^{\mathrm{rad}}$ & $\delta J_{z}^{\mathrm{ih}}$ & var & $\delta J_{y}^{\mathrm{rad}}$ & $\delta J_{y}^{\mathrm{ih}}$ & & $\delta J_{z}^{\mathrm{rad}}$ & $\delta J_{z}^{\mathrm{ih}}$ & \\
\hline SPTH0HP0 & .07505 & 07794 & 00052 & .00000 & 0.00854 & 0.00000 & 0.00000 & -0.00877 & 0.00000 & 0.49995 & 0.50437 & 0.00594 \\
\hline SPTH0P0 & 07463 & .07792 & .00042 & .00000 & 0.00936 & 0.00001 & 0.00000 & -0.00714 & 0.00001 & 0.49307 & 0.50346 & 0.00554 \\
\hline & 6357 & & 00039 & & & & & & & .41528 & 0.42519 & \\
\hline SPTH48P30 & 6139 & 06375 & 00036 & & -0.03910 & & & & & & & \\
\hline SPTH48P60 & 6083 & 06306 & 00035 & .08666 & -0.09718 & 0.00100 & 05186 & 0.04990 & .00130 & .40904 & 0.41370 & 00514 \\
\hline РТH48P90 & 05963 & 0.06169 & 00033 & .09907 & -0.08870 & 0.00167 & 0.00149 & 0.00777 & 0.00052 & .40571 & 0.41589 & 00499 \\
\hline SPTH48. & 6060 & 06255 & 00031 & & -0.09923 & & 574 & & & & & 497 \\
\hline SPTH48P150 & 06456 & 06704 & 00035 & .05578 & -0.04720 & & 341 & -0.10015 & 102 & & 2764 & 503 \\
\hline SPTH70P0 & .04970 & 0.05112 & .00029 & .00744 & -0.01082 & 0.00076 & 0.12241 & 0.13609 & .00158 & 0.32988 & 0.32970 & 0.00408 \\
\hline SPTH70P30 & & 05025 & 00027 & & & & & & & & & \\
\hline 60 & & & & & & & & & & & 224 & \\
\hline SPTH70 & 1889 & 05012 & & & 26 & & & 099 & & & & \\
\hline SPTH70P120 & 04972 & 05087 & 00026 & .09759 & 0004 & 165 & -0.06850 & -0.05769 & 026 & & 130 & 437 \\
\hline SPTH70P150 & 0.05469 & 0.05650 & 00032 & -0.05816 & 5297 & 33 & -0.1 & 2214 & 101 & 0.34928 & 5278 & 424 \\
\hline SPTH90P0 & & & & & & & & & & & & \\
\hline SPTH90 & & 04156 & 00021 & 87 & 25 & & & & & & & 363 \\
\hline SPTH90P60 & 03979 & 04069 & 00022 & .12369 & -0.1 & 076 & & & & & & 380 \\
\hline SPTH90P90 & & & & & & & & & & & & \\
\hline 120 & & & & & & & & & & & & \\
\hline SPTH90 & 1226 & 41 & & & & & & & & & & \\
\hline SPTH110P0 & 03476 & 561 & & & & & & & & & & \\
\hline SPTH110P30 & 3424 & & & & & & & & & & & 553 \\
\hline PTH1 & & & & & & & & & & & & \\
\hline 90 & & & & & & & & & & & & \\
\hline SPTH110P120 & & & & & & & & & & & & 66 \\
\hline SPTH110P150 & 3796 & 95 & & 74 & & & 264 & 278 & & & 19 & 555 \\
\hline SPTH132P0 & & & & & & & & & & & & \\
\hline SPTH13 & & & & & & & & & & & & 668 \\
\hline SPTH13 & & & & & & & & & & & & \\
\hline SPTH13 & & & & & & & & & & & & 599 \\
\hline SPTH1: & & & & & & & & & & & & \\
\hline SPTH1 & & & & & & & & & & & & 669 \\
\hline SPTH14 & & 807 & & & & & & & & & & 64 \\
\hline SPTH14 & 02737 & & & & -0 . & & & & & & & \\
\hline SPTH1 & & & & & & & & & & & & \\
\hline SPTH1 & & & & & & & & & & & & 986 \\
\hline SPTH14 & 02807 & 877 & & & -0.1 & & & & & & & 80 \\
\hline SPTH146P150 & & & & & & & & 327 & & & 58 & 69 \\
\hline SPTH180P0 & 02549 & 02612 & 031 & 00 & 443 & 000 & 00 & 0116 & 0000 & 3783 & 247 & 234 \\
\hline UD0.0C & & & & & & & & & & & & 301 \\
\hline UD0.0 & 03718 & & & & -0.00049 & & & & & & & 336 \\
\hline & & & & & & & & & & & & \\
\hline & 3744 & & & & & & & 0602 & & & & \\
\hline & 03732 & 0.03828 & .00010 & & & & & & & & 34 & 0352 \\
\hline & & 8 & & & & & & & & & & \\
\hline & & & & & & & & & & & & \\
\hline & & & & & & & & & & & 1571 & 0.00482 \\
\hline & & 0.03872 & 0.00015 & 0.00000 & -0.00616 & & & -0.00407 & & & 0.31574 & 0.00454 \\
\hline & 03642 & & 0.00015 & & & & & & 0.000 & & 0.31609 & 0.00393 \\
\hline & & & & & & & & & & & & \\
\hline UD0.85F & 0.03875 & 0.03906 & 0.00017 & 0.00000 & -0.00563 & 0.00000 & 0.00000 & -0.00473 & 0.00000 & 0.32005 & 0.31601 & 0.00467 \\
\hline
\end{tabular}


TABLE XIV: The magnitude of the ADM angular momentum $\left(J_{\mathrm{ADM}}\right)$ in units of $M^{2}$, final remnant angular momentum $\left(J_{\text {rem }}\right)$ in units of $M^{2}$, and the difference between the remnant and ADM angular momentum $\left(\delta J=\left|\vec{J}_{\mathrm{ADM}}-\vec{J}_{\text {rem }}\right|\right)$ in units of $M^{2}$, as well as the angle between $\vec{J}_{\mathrm{ADM}}$ and $\vec{J}_{\text {rem }}$, as calculated directly from the radiated angular momentum and the isolated horizon formalism.

\begin{tabular}{|c|c|c|c|c|c|c|c|c|c|}
\hline Conf & $J_{\mathrm{ADM}}$ & $J_{\mathrm{rem}}^{\mathrm{rad}}$ & $J_{\mathrm{rem}}^{\mathrm{ih}}$ & $\delta J^{\mathrm{rad}}$ & $\delta J^{\mathrm{ih}}$ & $\delta J^{\mathrm{rad}} / J_{\mathrm{ADM}}$ & $\delta J^{\mathrm{ih}} / J_{\mathrm{ADM}}$ & $\Theta^{\mathrm{rad}}$ & $\Theta^{\mathrm{ih}}$ \\
\hline SPTH0HPH0 & 1.27551 & 0.77556 & 0.77124 & 0.49995 & 0.50452 & 0.39196 & 0.39554 & 0.00000 & 0.90944 \\
\hline SPTHOPHO & 1.27551 & 0.78245 & 0.77214 & 0.49307 & 0.50360 & 0.38656 & 0.39482 & 0.00000 & 0.87359 \\
\hline SPTH48PH0 & 1.20006 & 0.77188 & 0.76281 & 0.42823 & 0.43755 & 0.35684 & 0.36460 & 0.38753 & 0.97019 \\
\hline SPTH48PH60 & 1.20006 & 0.77882 & 0.77223 & 0.42133 & 0.42788 & 0.35109 & 0.35655 & 0.50045 & 0.39885 \\
\hline SPTH48PH90 & 1.20006 & 0.78254 & 0.77560 & 0.41763 & 0.42532 & 0.34801 & 0.35441 & 0.56778 & 1.60150 \\
\hline SPTH48PH120 & 1.20006 & 0.77785 & 0.77176 & 0.42237 & 0.42836 & 0.35196 & 0.35695 & 0.68470 & 0.45136 \\
\hline SPTH70PH30 & 1.11517 & 0.76422 & 0.76039 & 0.35109 & 0.35519 & 0.31483 & 0.31850 & 0.63125 & 1.05622 \\
\hline SPTH70PH60 & 1.11517 & 0.76141 & 0.75798 & 0.35389 & 0.35826 & 0.31734 & 0.32126 & 0.60228 & 1.72674 \\
\hline SPTH70PH90 & 1.11517 & 0.76083 & 0.75849 & 0.35450 & 0.35714 & 0.31789 & 0.32025 & 0.67064 & 1.12619 \\
\hline SPTH70PH120 & 1.11517 & 0.75721 & 0.75507 & 0.35825 & 0.36031 & 0.32125 & 0.32309 & 0.90678 & 0.75903 \\
\hline SPTH70PH150 & 1.11517 & 0.74398 & 0.73858 & 0.37127 & 0.37706 & 0.33293 & 0.33812 & 0.48398 & 1.18693 \\
\hline SPTH90PH0 & 1.01827 & 0.72464 & 0.72096 & 0.29501 & 0.29972 & 0.28971 & 0.29434 & 1.90200 & 2.53544 \\
\hline SPTH90PH150 & 1.01827 & 0.71761 & 0.71384 & 0.30151 & 0.30467 & 0.29610 & 0.29921 & 1.51638 & 0.81087 \\
\hline SPTH110PH0 & 0.94224 & 0.65749 & 0.65426 & 0.28662 & 0.29273 & 0.30419 & 0.31067 & 2.38164 & 3.83364 \\
\hline SPTH110РH30 & 0.94224 & 0.65822 & 0.65518 & 0.28605 & 0.28926 & 0.30359 & 0.30699 & 2.47981 & 2.59825 \\
\hline SPTH110PH60 & 0.94224 & 0.65320 & 0.65093 & 0.29071 & 0.29548 & 0.30853 & 0.31359 & 2.27745 & 3.61911 \\
\hline SPTH110PH90 & 0.94224 & 0.65210 & 0.64985 & 0.29179 & 0.29429 & 0.30968 & 0.31233 & 2.26623 & 2.44657 \\
\hline SPTH110PH120 & 0.94224 & 0.65138 & 0.64942 & 0.29327 & 0.29561 & 0.31124 & 0.31373 & 2.74427 & 2.97197 \\
\hline SPTH110PH150 & 0.94224 & 0.64146 & 0.63906 & 0.30266 & 0.30510 & 0.32121 & 0.32381 & 2.48309 & 2.52897 \\
\hline SPTH132PH0 & 0.82423 & 0.55510 & 0.55252 & 0.27377 & 0.27579 & 0.33215 & 0.33461 & 4.25411 & 4.01621 \\
\hline SPTH132PH30 & 0.82423 & 0.55195 & 0.54806 & 0.27666 & 0.28126 & 0.33566 & 0.34124 & 4.16806 & 4.54273 \\
\hline SPTH132PH60 & 0.82423 & 0.55049 & 0.54742 & 0.27801 & 0.28583 & 0.33730 & 0.34679 & 4.13090 & 6.08258 \\
\hline SPTH132PH90 & 0.82423 & 0.54913 & 0.54699 & 0.27937 & 0.28174 & 0.33894 & 0.34183 & 4.14527 & 4.28525 \\
\hline SPTH180PH0 & 0.69562 & 0.40780 & 0.40318 & 0.28783 & 0.29251 & 0.41377 & 0.42050 & 0.00000 & 0.65079 \\
\hline
\end{tabular}


TABLE XV: The radiated mass and final remnant spin as measured using the IH formalism and from the radiation. The IH measurement proved to be more accurate as can be seen by comparing results from different resolutions.

\begin{tabular}{|c|c|c|c|c|}
\hline CONF. & $\delta \mathcal{M}^{\mathrm{IH}}$ & $\delta \mathcal{M}^{\mathrm{rad}}$ & $\alpha_{\mathrm{rem}}^{\mathrm{IH}}$ & $\alpha_{\mathrm{rem}}^{\mathrm{rad}}$ \\
\hline SPTH0PH0 & $0.08909 \pm 0.00007$ & $0.08584 \pm 0.00041$ & $0.90815 \pm 0.00070$ & $0.91374 \pm 0.00648$ \\
\hline SPTH0HPH0 & $0.08911 \pm 0.00003$ & $0.08625 \pm 0.00052$ & $0.90713 \pm 0.00047$ & $0.90652 \pm 0.00700$ \\
\hline SPTH48PH0 & $0.07713 \pm 0.00010$ & $0.07450 \pm 0.00037$ & $0.87488 \pm 0.00155$ & $0.88024 \pm 0.00543$ \\
\hline SPTH48PH30 & $0.07467 \pm 0.00010$ & $0.07233 \pm 0.00034$ & $0.87937 \pm 0.00135$ & $0.88376 \pm 0.00542$ \\
\hline SPTH48PH60 & $0.07399 \pm 0.00010$ & $0.07178 \pm 0.00034$ & $0.87968 \pm 0.00121$ & $0.88297 \pm 0.00554$ \\
\hline SPTH48PH90 & $0.07263 \pm 0.00010$ & $0.07060 \pm 0.00031$ & $0.88094 \pm 0.00120$ & $0.88493 \pm 0.00537$ \\
\hline SPTH48PH120 & $0.07346 \pm 0.00010$ & $0.07154 \pm 0.00029$ & $0.87819 \pm 0.00128$ & $0.88144 \pm 0.00532$ \\
\hline SPTH48PH150 & $0.07790 \pm 0.00009$ & $0.07545 \pm 0.00033$ & $0.87140 \pm 0.00137$ & $0.87542 \pm 0.00542$ \\
\hline SPTH70PH0 & $0.06178 \pm 0.00001$ & $0.06037 \pm 0.00028$ & $0.84291 \pm 0.00006$ & $\overline{0.84528 \pm 0.00431}$ \\
\hline SPTH70PH30 & $0.06096 \pm 0.00001$ & $0.05965 \pm 0.00026$ & $0.84298 \pm 0.00010$ & $0.84488 \pm 0.00437$ \\
\hline SPTH70PH60 & $0.06120 \pm 0.00001$ & $0.05987 \pm 0.00027$ & $0.84075 \pm 0.00006$ & $0.84217 \pm 0.00459$ \\
\hline SPTH70PH90 & $0.06081 \pm 0.00001$ & $0.05959 \pm 0.00027$ & $0.84065 \pm 0.00004$ & $0.84105 \pm 0.00471$ \\
\hline SPTH70PH120 & $0.06153 \pm 0.00001$ & $0.06039 \pm 0.00026$ & $0.83818 \pm 0.00004$ & $0.83852 \pm 0.00459$ \\
\hline SPTH70PH150 & $0.06708 \pm 0.00001$ & $0.06529 \pm 0.00031$ & $0.82968 \pm 0.00005$ & $0.83256 \pm 0.00450$ \\
\hline SPTH90PH0 & $0.05181 \pm 0.00000$ & $0.05087 \pm 0.00022$ & $0.78461 \pm 0.00002$ & $0.78705 \pm 0.00361$ \\
\hline SPTH90РH30 & $0.05199 \pm 0.00000$ & $0.05108 \pm 0.00021$ & $0.78262 \pm 0.00002$ & $0.78433 \pm 0.00372$ \\
\hline SPTH90PH60 & $0.05115 \pm 0.00000$ & $0.05026 \pm 0.00021$ & $0.78277 \pm 0.00002$ & $0.78397 \pm 0.00386$ \\
\hline SPTH90РH90 & $0.05121 \pm 0.00000$ & $0.05040 \pm 0.00022$ & $0.78096 \pm 0.00019$ & $0.78200 \pm 0.00398$ \\
\hline SPTH90PH120 & $0.05511 \pm 0.00000$ & $0.05413 \pm 0.00024$ & $0.77164 \pm 0.00001$ & $0.77261 \pm 0.00391$ \\
\hline SPTH90PH150 & $0.05378 \pm 0.00000$ & $0.05264 \pm 0.00026$ & $0.78009 \pm 0.00001$ & $0.78233 \pm 0.00370$ \\
\hline SPTH110PH0 & $0.04457 \pm 0.00000$ & $0.04373 \pm 0.00025$ & $0.70347 \pm 0.00000$ & $0.70570 \pm 0.00550$ \\
\hline SPTH110РH30 & $0.04406 \pm 0.00000$ & $0.04325 \pm 0.00024$ & $0.70365 \pm 0.00000$ & $0.70572 \pm 0.00559$ \\
\hline SPTH110PH60 & $0.04475 \pm 0.00000$ & $0.04389 \pm 0.00024$ & $0.70003 \pm 0.00000$ & $0.70122 \pm 0.00576$ \\
\hline SPTH110PH90 & $0.04465 \pm 0.00000$ & $0.04384 \pm 0.00024$ & $0.69873 \pm 0.00001$ & $0.69996 \pm 0.00591$ \\
\hline SPTH110PH120 & $0.04485 \pm 0.00000$ & $0.04408 \pm 0.00024$ & $0.69862 \pm 0.00000$ & $0.69960 \pm 0.00572$ \\
\hline SPTH110PH150 & $0.04790 \pm 0.00000$ & $0.04692 \pm 0.00026$ & $0.69191 \pm 0.00000$ & $0.69308 \pm 0.00561$ \\
\hline SPTH132PH0 & $0.03915 \pm 0.00000$ & $0.03846 \pm 0.00024$ & $0.58826 \pm 0.00000$ & $0.59016 \pm 0.00678$ \\
\hline SPTH132PH30 & $0.03982 \pm 0.00000$ & $0.03908 \pm 0.00023$ & $0.58431 \pm 0.00000$ & $0.58756 \pm 0.00682$ \\
\hline SPTH132PH60 & $0.03980 \pm 0.00000$ & $0.03904 \pm 0.00023$ & $0.58358 \pm 0.00000$ & $0.58591 \pm 0.00698$ \\
\hline SPTH132PH90 & $0.03971 \pm 0.00000$ & $0.03896 \pm 0.00023$ & $0.58300 \pm 0.00000$ & $0.58436 \pm 0.00712$ \\
\hline SPTH132PH120 & $0.03938 \pm 0.00000$ & $0.03861 \pm 0.00023$ & $0.58512 \pm 0.00000$ & $0.58754 \pm 0.00697$ \\
\hline SPTH132PH150 & $0.03825 \pm 0.00000$ & $0.03753 \pm 0.00024$ & $0.59121 \pm 0.00000$ & $0.59360 \pm 0.00678$ \\
\hline SPTH146PH0 & $0.03578 \pm 0.00000$ & $0.03513 \pm 0.00029$ & $0.51828 \pm 0.00000$ & $0.51948 \pm 0.00995$ \\
\hline SPTH146РH30 & $0.03573 \pm 0.00000$ & $0.03509 \pm 0.00028$ & $0.51878 \pm 0.00000$ & $0.51931 \pm 0.01004$ \\
\hline SPTH146PH60 & $0.03580 \pm 0.00000$ & $0.03514 \pm 0.00029$ & $0.51778 \pm 0.00001$ & $0.51765 \pm 0.01015$ \\
\hline SPTH146PH90 & $0.03592 \pm 0.00000$ & $0.03523 \pm 0.00029$ & $0.51627 \pm 0.00000$ & $0.51661 \pm 0.01019$ \\
\hline SPTH146PH120 & $0.03648 \pm 0.00000$ & $0.03578 \pm 0.00029$ & $0.51459 \pm 0.00000$ & $0.51448 \pm 0.01014$ \\
\hline SPTH146PH150 & $0.03642 \pm 0.00000$ & $0.03575 \pm 0.00029$ & $0.51570 \pm 0.00001$ & $0.51577 \pm 0.01000$ \\
\hline SPTH180PH0 & $0.03339 \pm 0.00000$ & $0.03277 \pm 0.00031$ & $0.42509 \pm 0.00000$ & $0.42941 \pm 0.01299$ \\
\hline UD0.00C & $0.04833 \pm 0.00000$ & $0.04811 \pm 0.00006$ & $0.68628 \pm 0.00000$ & $0.68247 \pm 0.00426$ \\
\hline UD0.00M & $0.04833 \pm 0.00000$ & $0.04832 \pm 0.00011$ & $0.68629 \pm 0.00000$ & $0.68185 \pm 0.00450$ \\
\hline UD0.60C & $0.04862 \pm 0.00000$ & $0.04832 \pm 0.00012$ & $0.68498 \pm 0.00000$ & $0.68113 \pm 0.00462$ \\
\hline UD0.60M & $0.04862 \pm 0.00000$ & $0.04855 \pm 0.00015$ & $0.68498 \pm 0.00000$ & $0.68048 \pm 0.00481$ \\
\hline UD0.70C & $0.04874 \pm 0.00000$ & $0.04835 \pm 0.00015$ & $0.68450 \pm 0.00000$ & $\overline{0.68072 \pm 0.00480}$ \\
\hline UD0.70M & $0.04872 \pm 0.00000$ & $0.04857 \pm 0.00018$ & $0.68451 \pm 0.00000$ & $0.68006 \pm 0.00501$ \\
\hline UD0.80C & $0.04881 \pm 0.00000$ & $0.04819 \pm 0.00020$ & $0.68358 \pm 0.00002$ & $0.68199 \pm 0.00495$ \\
\hline UD0.80M & $0.04887 \pm 0.00000$ & $0.04856 \pm 0.00022$ & $0.68400 \pm 0.00000$ & $0.67943 \pm 0.00522$ \\
\hline UD0.80F & $0.04886 \pm 0.00000$ & $0.04865 \pm 0.00015$ & $0.68393 \pm 0.00000$ & $0.67947 \pm 0.00492$ \\
\hline UD0.85C & $0.04804 \pm 0.00008$ & $0.04759 \pm 0.00023$ & $0.68169 \pm 0.00010$ & $0.68838 \pm 0.00528$ \\
\hline UD0.85M & $0.04885 \pm 0.00003$ & $0.04834 \pm 0.00023$ & $0.68311 \pm 0.00004$ & $0.68170 \pm 0.00521$ \\
\hline UD0.85F & $0.04895 \pm 0.00000$ & $0.04862 \pm 0.00017$ & $0.68371 \pm 0.00000$ & $0.67885 \pm 0.00506$ \\
\hline
\end{tabular}


TABLE XVI: The recoil velocity for the UD configurations in units of $\mathrm{km} \mathrm{s}^{-1}$. Note that magnitude of the recoil is in good agreement between resolutions.

\begin{tabular}{llll}
\hline \hline Conf & $V_{x}$ & $V_{y}$ & $V$ \\
\hline UD0.60C & $-51.69 \pm 2.72$ & $-271.61 \pm 1.71$ & $276.49 \pm 1.75$ \\
UD0.60M & $-50.04 \pm 2.26$ & $-274.30 \pm 2.07$ & $278.82 \pm 2.07$ \\
\hline UD0.70C & $-96.65 \pm 2.90$ & $-307.53 \pm 1.23$ & $322.36 \pm 1.46$ \\
UD0.70M & $-96.86 \pm 2.74$ & $-310.38 \pm 1.26$ & $325.15 \pm 1.45$ \\
\hline UD0.80C & $-244.46 \pm 3.70$ & $-273.38 \pm 0.18$ & $366.74 \pm 2.47$ \\
UD0.80M & $-144.65 \pm 3.74$ & $-341.98 \pm 0.94$ & $371.31 \pm 1.69$ \\
UD0.80F & $-160.89 \pm 3.18$ & $-336.12 \pm 1.25$ & $372.65 \pm 1.78$ \\
\hline UD0.85C & $-250.52 \pm 4.25$ & $-314.66 \pm 1.01$ & $402.21 \pm 2.76$ \\
UD0.85M & $-307.15 \pm 3.53$ & $-242.73 \pm 0.12$ & $391.48 \pm 2.77$ \\
UD0.85F & $-171.48 \pm 3.65$ & $-357.11 \pm 1.76$ & $396.14 \pm 2.24$ \\
\hline \hline
\end{tabular}

